Cochrane Database of Systematic Reviews

\title{
Transcutaneous electrical nerve stimulation (TENS) for fibromyalgia in adults (Review)
}

Johnson MI, Claydon LS, Herbison GP, Jones G, Paley CA

Johnson MI, Claydon LS, Herbison GP, Jones G, Paley CA.

Transcutaneous electrical nerve stimulation (TENS) for fibromyalgia in adults.

Cochrane Database of Systematic Reviews 2017, Issue 10. Art. No.: CD012172.

DOI: 10.1002/14651858.CD012172.pub2.

www.cochranelibrary.com 
HEADER 1

ABSTRACT

PLAIN LANGUAGE SUMMARY

SUMMARY OF FINDINGS

BACKGROUND

OBJECTIVES

METHODS

RESULTS

Figure 1.

Figure 2.

Figure 3.

DISCUSSION

AUTHORS' CONCLUSIONS

ACKNOWLEDGEMENTS

REFERENCES

CHARACTERISTICS OF STUDIES

ADDITIONAL TABLES

APPENDICES

WHAT'S NEW

.......................

CONTRIBUTIONS OF AUTHORS

DECLARATIONS OF INTEREST

DIFFERENCES BETWEEN PROTOCOL AND REVIEW

NOTES

INDEX TERMS

\section{TABLE OF CONTENTS}


[Intervention Review]

\section{Transcutaneous electrical nerve stimulation (TENS) for fibromyalgia in adults}

Mark I Johnson ${ }^{1}$, Leica S Claydon², G Peter Herbison ${ }^{3}$, Gareth Jones ${ }^{1}$, Carole A Paley ${ }^{4}$

1Faculty of Health and Social Sciences, Leeds Beckett University, Leeds, UK. 2Anglia Ruskin University, Postgraduate Medical Institute, Chelmsford, UK. ${ }^{3}$ Department of Preventive \& Social Medicine, Dunedin School of Medicine, University of Otago, Dunedin, New Zealand.

${ }^{4}$ Research \& Development Department, Airedale NHS Foundation Trust, Keighley, UK

Contact address: Mark I Johnson, Faculty of Health and Social Sciences, Leeds Beckett University, City Campus, Calverley Street, Leeds, LS1 3HE, UK. M.Johnson@LeedsBeckett.ac.uk.

Editorial group: Cochrane Pain, Palliative and Supportive Care Group.

Publication status and date: Stable (no update expected for reasons given in 'What's new'), published in Issue 10, 2017.

Citation: Johnson MI, Claydon LS, Herbison GP, Jones G, Paley CA. Transcutaneous electrical nerve stimulation (TENS) for fibromyalgia in adults. Cochrane Database of Systematic Reviews 2017, Issue 10. Art. No.: CD012172. DOI: 10.1002/14651858.CD012172.pub2.

Copyright @ 2017 The Cochrane Collaboration. Published by John Wiley \& Sons, Ltd.

\section{A B S T R A C T}

\section{Background}

Fibromyalgia is characterised by persistent, widespread pain; sleep problems; and fatigue. Transcutaneous electrical nerve stimulation (TENS) is the delivery of pulsed electrical currents across the intact surface of the skin to stimulate peripheral nerves and is used extensively to manage painful conditions. TENS is inexpensive, safe, and can be self-administered. TENS reduces pain during movement in some people so it may be a useful adjunct to assist participation in exercise and activities of daily living. To date, there has been only one systematic review in 2012 which included TENS, amongst other treatments, for fibromyalgia, and the authors concluded that TENS was not effective.

\section{Objectives}

To assess the analgesic efficacy and adverse events of TENS alone or added to usual care (including exercise) compared with placebo (sham) TENS; no treatment; exercise alone; or other treatment including medication, electroacupuncture, warmth therapy, or hydrotherapy for fibromyalgia in adults.

\section{Search methods}

We searched the following electronic databases up to 18 January 2017: CENTRAL (CRSO); MEDLINE (Ovid); Embase (Ovid); CINAHL (EBSCO); PsycINFO (Ovid); LILACS; PEDRO; Web of Science (ISI); AMED (Ovid); and SPORTDiscus (EBSCO). We also searched three trial registries. There were no language restrictions.

\section{Selection criteria}

We included randomised controlled trials (RCTs) or quasi-randomised trials of TENS treatment for pain associated with fibromyalgia in adults. We included cross-over and parallel-group trial designs. We included studies that evaluated TENS administered using non-invasive techniques at intensities that produced perceptible TENS sensations during stimulation at either the site of pain or over nerve bundles proximal (or near) to the site of pain. We included TENS administered as a sole treatment or TENS in combination with other treatments, and TENS given as a single treatment or as a course of treatments.

\section{Data collection and analysis}

Two review authors independently determined study eligibility by assessing each record and reaching agreement by discussion. A third review author acted as arbiter. We did not anonymise the records of studies before assessment. Two review authors independently extracted data and assessed risk of bias of included studies before entering information into a 'Characteristics of included studies' table. 
Primary outcomes were participant-reported pain relief from baseline of $30 \%$ or greater or $50 \%$ or greater, and Patient Global Impression of Change (PGIC). We assessed the evidence using GRADE and added 'Summary of findings' tables.

\section{Main results}

We included eight studies (seven RCTs, one quasi-RCT, 315 adults (299 women), aged 18 to 75 years): six used a parallel-group design and two used a cross-over design. Sample sizes of intervention arms were five to 43 participants.

Two studies, one of which was a cross-over design, compared TENS with placebo TENS (82 participants), one study compared TENS with no treatment (43 participants), and four studies compared TENS with other treatments (medication (two studies, 74 participants), electroacupuncture (one study, 44 participants), superficial warmth (one cross-over study, 32 participants), and hydrotherapy (one study, 10 participants)). Two studies compared TENS plus exercise with exercise alone (98 participants, 49 per treatment arm). None of the studies measured participant-reported pain relief of $50 \%$ or greater or PGIC. Overall, the studies were at unclear or high risk of bias, and in particular all were at high risk of bias for sample size.

Only one study (14 participants) measured the primary outcome participant-reported pain relief of 30\% or greater. Thirty percent achieved $30 \%$ or greater reduction in pain with TENS and exercise compared with $13 \%$ with exercise alone. One study found $10 / 28$ participants reported pain relief of $25 \%$ or greater with TENS compared with $10 / 24$ participants using superficial warmth $\left(42{ }^{\circ} \mathrm{C}\right)$. We judged that statistical pooling was not possible because there were insufficient data and outcomes were not homogeneous.

There were no data for the primary outcomes participant-reported pain relief from baseline of $50 \%$ or greater and PGIC.

There was a paucity of data for secondary outcomes. One pilot cross-over study of 43 participants found that the mean (95\% confidence intervals $(\mathrm{CI})$ ) decrease in pain intensity on movement (100-mm visual analogue scale (VAS)) during one 30 -minute treatment was $11.1 \mathrm{~mm}$ $(95 \% \mathrm{Cl} 5.9$ to 16.3$)$ for TENS and $2.3 \mathrm{~mm}(95 \% \mathrm{Cl} 2.4$ to 7.7$)$ for placebo TENS. There were no significant differences between TENS and placebo for pain at rest. One parallel group study of 39 participants found that mean \pm standard deviation (SD) pain intensity (100-mm VAS) decreased from $85 \pm 20 \mathrm{~mm}$ at baseline to $43 \pm 20 \mathrm{~mm}$ after one week of dual-site TENS; decreased from $85 \pm 10 \mathrm{~mm}$ at baseline to $60 \pm$ $10 \mathrm{~mm}$ after single-site TENS; and decreased from $82 \pm 20 \mathrm{~mm}$ at baseline to $80 \pm 20 \mathrm{~mm}$ after one week of placebo TENS. The authors of seven studies concluded that TENS relieved pain but the findings of single small studies are unlikely to be correct.

One study found clinically important improvements in Fibromyalgia Impact Questionnaire (FIQ) subscales for work performance, fatigue, stiffness, anxiety, and depression for TENS with exercise compared with exercise alone. One study found no additional improvements in FIQ scores when TENS was added to the first three weeks of a 12-week supervised exercise programme.

No serious adverse events were reported in any of the studies although there were reports of TENS causing minor discomfort in a total of 3 participants.

The quality of evidence was very low. We downgraded the GRADE rating mostly due to a lack of data; therefore, we have little confidence in the effect estimates where available.

\section{Authors' conclusions}

There was insufficient high-quality evidence to support or refute the use of TENS for fibromyalgia. We found a small number of inadequately powered studies with incomplete reporting of methodologies and treatment interventions.

\section{PLAIN LANGUAGE SUMMARY}

\section{TENS for fibromyalgia in adults}

\section{Review question}

Does transcutaneous electrical nerve stimulation (TENS) relieve pain in adults with fibromyalgia?

\section{Background}

Fibromyalgia is a long-term medical condition that is characterised by long-lasting widespread pain throughout the body. TENS is a treatment that involves putting pulsed electrical currents across the surface of the skin using two or four electrodes. It is used to manage painful conditions. TENS is inexpensive, can be self-administered by people with fibromyalgia, and is not associated with any particular side effects. TENS reduces pain during movement so it may be useful in addition to other treatments to help people carry on their normal lives.

\section{Study characteristics}

In January 2017, we found eight clinical studies that examined 315 people. We included TENS administered to produce a non-painful 'tingling' sensation at the site of pain either as a treatment alone or combined with exercise treatment. All studies used TENS in comparison with 'fake' (called placebo or sham) TENS, no treatment, or other treatments such as medicine or hydrotherapy (treatment in water). 


\section{Key results}

We did not find enough high-quality studies to allow us to come to any conclusions about the effectiveness of TENS for fibromyalgia pain. Even though seven studies concluded that TENS relieved pain associated with fibromyalgia, the studies were low quality and the findings for measures of pain were inconsistently reported. Studies did not measure most of our outcomes and it was not always clear what aspects of pain were being reported (e.g. present pain, remembered pain, pain severity, etc.). Only one small pilot study found that one 30 -minute treatment of TENS reduced pain on movement during and immediately after treatment; however, there were too few participants observed and it is unknown whether this effect would be maintained over a longer course of TENS treatments. Overall, it is not possible to judge whether TENS reduces pain associated with fibromyalgia. There were no serious side events reported in any of the studies.

\section{Quality of the evidence}

We rated the quality of the evidence from studies using four levels: very low, low, moderate, or high. Very low-quality evidence means that we are very uncertain about the results. High-quality evidence means that we are very confident in the results. The quality of the evidence was very low overall because of a lack of data. 


\section{SUMMARY OF FINDINGS}

\section{Summary of findings for the main comparison. TENS compared to placebo TENS for fibromyalgia}

\section{TENS compared to placebo TENS for fibromyalgia}

Patient or population: adults with fibromyalgia

Setting: hospital and university clinic

Intervention: TENS

Comparison: placebo TENS

\begin{tabular}{|c|c|c|c|c|c|c|}
\hline Outcomes & $\begin{array}{l}\text { Probable outcome with } \\
\text { TENS }\end{array}$ & $\begin{array}{l}\text { Probable outcome with place- } \\
\text { bo TENS }\end{array}$ & $\begin{array}{l}\text { Relative effect } \\
(95 \% \mathrm{Cl})\end{array}$ & $\begin{array}{l}\text { No of partici- } \\
\text { pants } \\
\text { (studies) }\end{array}$ & $\begin{array}{l}\text { Quality of the } \\
\text { evidence } \\
\text { (GRADE) }\end{array}$ & Comments \\
\hline $\begin{array}{l}\text { Participant-reported pain } \\
\text { relief } \geq \mathbf{3 0} \% \text { ( } \geq 30 \% \text { pain re- } \\
\text { lief) }\end{array}$ & No data & No data & Not calculated & No data & $\begin{array}{l}\oplus \ominus \ominus \ominus \\
\text { Very low }\end{array}$ & No data \\
\hline $\begin{array}{l}\text { Participant-reported pain } \\
\text { relief } \geq \mathbf{5 0} \% \text { ( } \geq 50 \% \text { pain re- } \\
\text { lief) }\end{array}$ & No data & No data & Not calculated & No data & $\begin{array}{l}\oplus \ominus \ominus \ominus \\
\text { Very low }\end{array}$ & No data \\
\hline PGIC very much improved & No data & No data & Not calculated & No data & $\begin{array}{l}\oplus \ominus \ominus \ominus \\
\text { Very low }\end{array}$ & No data \\
\hline $\begin{array}{l}\text { PGIC much or very much } \\
\text { improved }\end{array}$ & No data & No data & Not calculated & No data & $\begin{array}{l}\oplus \ominus \ominus \ominus \\
\text { Very low }\end{array}$ & No data \\
\hline $\begin{array}{l}\text { Withdrawals due to ad- } \\
\text { verse events }\end{array}$ & \multicolumn{2}{|c|}{$\begin{array}{l}\text { In a cross-over study, Dailey } 2013 \text { reported } 2 \text { withdrawals af- } \\
\text { ter the no-TENS intervention (without reasons) resulting in } \\
\text { missing data from the TENS and placebo TENS interventions. } \\
\text { Lauretti } 2013 \text { reported } 2 \text { withdrawals from the placebo TENS } \\
\text { group and } 1 \text { withdrawal from the TENS group due to absence of } \\
\text { symptom relief. }\end{array}$} & Not calculated & $\begin{array}{l}54 \text { participants } \\
\text { per treatment } \\
\text { arm } \\
\text { ( } 2 \text { RCTs) }\end{array}$ & $\begin{array}{l}\oplus \ominus \ominus \ominus \\
\text { Very low }\end{array}$ & $\begin{array}{l}\text { Downgraded by } \\
3 \text { levels due to } \\
\text { small number } \\
\text { of studies, par- } \\
\text { ticipants, and } \\
\text { events. }\end{array}$ \\
\hline
\end{tabular}

${ }^{\star}$ The risk in the intervention group (and its 95\% confidence interval) is based on the assumed risk in the comparison group and the relative effect of the intervention (and its $95 \% \mathrm{Cl}$ ).

CI: confidence interval; PGIC: Patient Global Impression of Change; RCT: randomised controlled trial; TENS: transcutaneous electrical nerve stimulation.

\section{GRADE Working Group grades of evidence}


Moderate quality: We are moderately confident in the effect estimate: The true effect is likely to be close to the estimate of the effect, but there is a possibility that it is substantially different

Low quality: Our confidence in the effect estimate is limited: The true effect may be substantially different from the estimate of the effect.

Very low quality: We have very little confidence in the effect estimate: The true effect is likely to be substantially different from the estimate of effect.

Summary of findings 2 . TENS compared to no treatment for fibromyalgia

TENS compared to no treatment for fibromyalgia

Patient or population: adults with fibromyalgia

Setting: university clinic

Intervention: TENS

Comparison: no treatment

\begin{tabular}{|c|c|c|c|c|c|c|}
\hline Outcomes & $\begin{array}{l}\text { Probably outcome with } \\
\text { TENS }\end{array}$ & $\begin{array}{l}\text { Probable outcome with } \\
\text { no treatment }\end{array}$ & $\begin{array}{l}\text { Relative effect } \\
(95 \% \mathrm{Cl})\end{array}$ & $\begin{array}{l}\text { No of partici- } \\
\text { pants } \\
\text { (studies) }\end{array}$ & $\begin{array}{l}\text { Quality of the } \\
\text { evidence } \\
\text { (GRADE) }\end{array}$ & Comments \\
\hline $\begin{array}{l}\text { Participant-reported pain relief } \geq \\
\mathbf{3 0} \% \text { ( } \geq 30 \% \text { pain relief) }\end{array}$ & No data & No data & Not calculated & No data & $\begin{array}{l}\oplus \ominus \ominus \ominus \\
\text { Very low }\end{array}$ & No data \\
\hline $\begin{array}{l}\text { Participant-reported pain relief } \geq \\
\mathbf{5 0} \% \text { ( } \geq 50 \% \text { pain relief) }\end{array}$ & No data & No data & Not calculated & No data & $\begin{array}{l}\oplus \ominus \ominus \ominus \\
\text { Very low }\end{array}$ & No data \\
\hline $\begin{array}{l}\text { PGIC much or very much im- } \\
\text { proved }\end{array}$ & No data & No data & Not calculated & No data & $\begin{array}{l}\oplus \ominus \ominus \ominus \\
\text { Very low }\end{array}$ & No data \\
\hline PGIC very much improved & No data & No data & Not calculated & No data & $\begin{array}{l}\oplus \ominus \ominus \ominus \\
\text { Very low }\end{array}$ & No data \\
\hline $\begin{array}{l}\text { Withdrawals due to adverse } \\
\text { events }\end{array}$ & $\begin{array}{l}\text { In a cross-over study, Dail } \\
\text { were } 2 \text { withdrawals after } \\
\text { (without reasons) resultin } \\
\text { TENS and placebo TENS }\end{array}$ & $\begin{array}{l}\text { y } 2013 \text { reported that there } \\
\text { le no-TENS intervention } \\
\text { in missing data from the } \\
\text { terventions. }\end{array}$ & Not calculated & No data & $\begin{array}{l}\oplus \ominus \ominus \ominus \\
\text { Very low }\end{array}$ & $\begin{array}{l}\text { Downgraded by } \\
3 \text { levels due to } \\
\text { small number } \\
\text { of studies, par- } \\
\text { ticipants, and } \\
\text { events. }\end{array}$ \\
\hline
\end{tabular}

${ }^{\star}$ The risk in the intervention group (and its $95 \%$ confidence interval) is based on the assumed risk in the comparison group and the relative effect of the intervention (and its $95 \% \mathrm{Cl})$.

CI: confidence interval; PGIC: Patient Global Impression of Change; RCT: randomised controlled trial; TENS: transcutaneous electrical nerve stimulation. 
GRADE Working Group grades of evidence

High quality: We are very confident that the true effect lies close to that of the estimate of the effect.

Moderate quality: We are moderately confident in the effect estimate: The true effect is likely to be close to the estimate of the effect, but there is a possibility that it is substantially different.

Low quality: Our confidence in the effect estimate is limited: The true effect may be substantially different from the estimate of the effect.

Very low quality: We have very little confidence in the effect estimate: The true effect is likely to be substantially different from the estimate of effect.

\section{Summary of findings 3 . TENS with exercise compared to exercise alone for fibromyalgia}

\section{TENS with exercise compared to exercise alone for fibromyalgia}

Patient or population: adults with fibromyalgia

Setting: hospital and university clinic

Intervention: TENS with exercise

Comparison: exercise on its own

\begin{tabular}{|c|c|c|c|c|c|c|}
\hline Outcomes & $\begin{array}{l}\text { Probably outcome with TENS } \\
\text { with exercise }\end{array}$ & $\begin{array}{l}\text { Probable outcome with exercise } \\
\text { on its own }\end{array}$ & $\begin{array}{l}\text { Relative effect } \\
(95 \% \mathrm{CI})\end{array}$ & $\begin{array}{l}\text { No of partici- } \\
\text { pants } \\
\text { (studies) }\end{array}$ & $\begin{array}{l}\text { Quality of the } \\
\text { evidence } \\
\text { (GRADE) }\end{array}$ & Comments \\
\hline $\begin{array}{l}\text { Participant-re- } \\
\text { ported pain re- } \\
\text { lief } \geq \mathbf{3 0} \% \text { ( } \geq 30 \% \\
\text { pain relief) }\end{array}$ & \multicolumn{2}{|c|}{$\begin{array}{l}\text { Carbonario } 2013 \text { reported that } 30 \% \text { of } 14 \text { participants in the TENS with ex- } \\
\text { ercise group achieved } \geq 30 \% \text { reduction in pain and this was significantly } \\
\text { greater than } 13 \% \text { of } 14 \text { participants in the exercise without TENS group. } \\
\text { However, these percentages equate to } 4.2 \text { participants and } 1.82 \text { partici- } \\
\text { pants respectively, which is illogical. The mean } \pm \text { SD reduction in pain in- } \\
\text { tensity was } 20 \pm 29 \mathrm{~mm} \text { for TENS and } 7.0 \pm 37 \mathrm{~mm} \text { for the exercise without } \\
\text { TENS. }\end{array}$} & Not calculated & $\begin{array}{l}28 \\
(1 \mathrm{RCT})\end{array}$ & $\begin{array}{l}\oplus \ominus \odot \odot \\
\text { Very low }\end{array}$ & $\begin{array}{l}\text { Downgraded by } \\
3 \text { levels due to } \\
\text { small number } \\
\text { of studies, par- } \\
\text { ticipants, and } \\
\text { events }\end{array}$ \\
\hline $\begin{array}{l}\text { Participant-re- } \\
\text { ported pain re- } \\
\text { lief } \geq \mathbf{5 0} \% \text { ( } \geq 50 \% \\
\text { pain relief) }\end{array}$ & No data & No data & Not calculated & No data & $\begin{array}{l}\oplus \ominus \ominus \ominus \\
\text { Very lowLOW }\end{array}$ & No data \\
\hline $\begin{array}{l}\text { PGIC much or } \\
\text { very much im- } \\
\text { proved }\end{array}$ & No data & No data & Not calculated & No data & $\begin{array}{l}\oplus \ominus \ominus \ominus \\
\text { Very low }\end{array}$ & No data \\
\hline $\begin{array}{l}\text { PGIC very much } \\
\text { improved }\end{array}$ & No data & No data & Not calculated & No data & $\begin{array}{l}\oplus \ominus \ominus \ominus \\
\text { Very low }\end{array}$ & No data \\
\hline
\end{tabular}




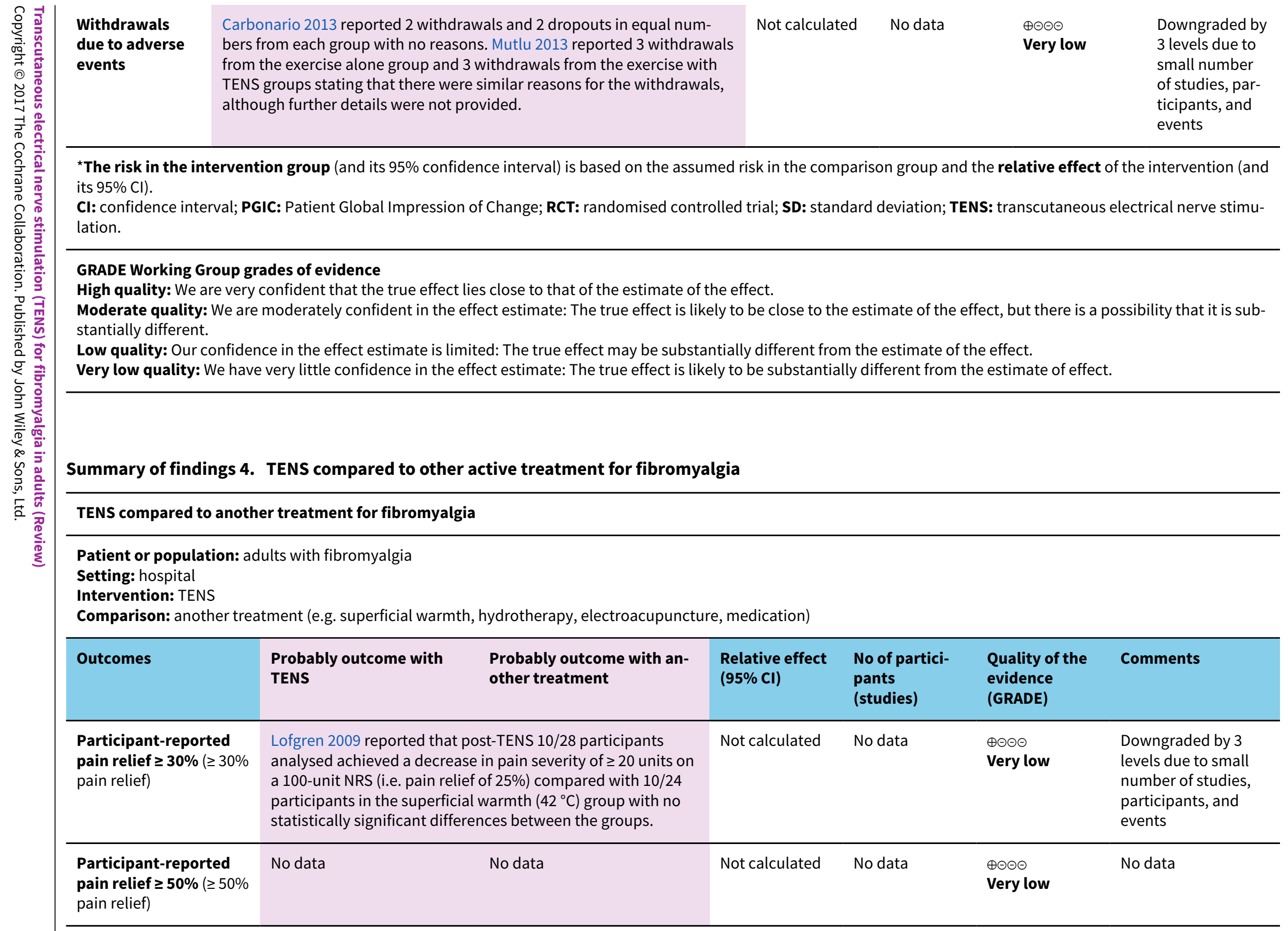




\begin{tabular}{|c|c|c|c|c|c|}
\hline $\begin{array}{l}\text { PGIC much or very } \\
\text { much improved }\end{array}$ & No data & Not calculated & No data & $\begin{array}{l}\oplus \ominus \ominus \ominus \\
\text { Very low }\end{array}$ & No data \\
\hline $\begin{array}{l}\text { PGIC very much im- } \\
\text { proved }\end{array}$ & No data & Not calculated & No data & $\begin{array}{l}\oplus \ominus \ominus \ominus \\
\text { Very low }\end{array}$ & No data \\
\hline $\begin{array}{l}\text { Withdrawals due to ad- } \\
\text { verse events }\end{array}$ & $\begin{array}{l}\text { Lofgren } 2009 \text { reported that } 2 \text { participants reported increased } \\
\text { pain during TENS. }\end{array}$ & Not calculated & No data & $\begin{array}{l}\oplus \ominus \ominus \ominus \\
\text { Very low }\end{array}$ & $\begin{array}{l}\text { Downgraded by } 3 \\
\text { levels due to small } \\
\text { number of studies, } \\
\text { participants, and } \\
\text { events }\end{array}$ \\
\hline
\end{tabular}

${ }^{*}$ The risk in the intervention group (and its $95 \%$ confidence interval) is based on the assumed risk in the comparison group and the relative effect of the intervention (and its $95 \% \mathrm{Cl}$ ).

CI: confidence interval; NRS: numerical rating scale; PGIC: Patient Global Impression of Change; TENS: transcutaneous electrical nerve stimulation.

\section{GRADE Working Group grades of evidence}

High quality: We are very confident that the true effect lies close to that of the estimate of the effect.

Moderate quality: We are moderately confident in the effect estimate: The true effect is likely to be close to the estimate of the effect, but there is a possibility that it is substantially different.

Low quality: Our confidence in the effect estimate is limited: The true effect may be substantially different from the estimate of the effect.

Very low quality: We have very little confidence in the effect estimate: The true effect is likely to be substantially different from the estimate of effect. 


\section{B A C K G R O U N D}

This review is based on a template for reviews of drugs used to relieve fibromyalgia. The aim is for all reviews to use the same methods, based on new criteria for what constitutes reliable evidence in chronic pain (Moore 2010a; Appendix 1).

\section{Description of the condition}

Fibromyalgia is a long-term medical condition that is characterised by chronic widespread pain in the muscles and joints, with sensitivity to pressure stimuli. The symptoms may vary from person to person, but the main symptom is widespread pain throughout the body. This may be worse in certain areas, such as the back or neck. Pain may be described as aching, burning, stabbing, or sharp and may be accompanied by hyperalgesia (heightened sensitivity to pain) and allodynia (pain on very mild stimulus). Pain is often continuous but it may fluctuate in severity depending on various factors including stress, physical activity, and the weather. Exposure to certain environmental stimuli (e.g. smoke, certain foods, and bright lights) may cause flare-ups. Other presenting symptoms may include stiffness, especially in the morning; muscle spasm; depression; fatigue; poor sleep quality, including non-restorative sleep; cognitive difficulties in thinking, learning, attention, and concentration; headaches, including severe migraines; and irritable bowel syndrome (Wolfe 2014). Originally, the American College of Rheumatology classification criteria for fibromyalgia were widespread pain (axial pain, leftand right-sided pain, upper and lower segment pain) that lasts for longer than three months, with pain on palpation at 11 or more of 18 specified tender points (Wolfe 1990). More recently, a definition of fibromyalgia has been proposed based on symptom severity and the presence of widespread pain, which does not require palpation of tender points for diagnosis (Wolfe 2010). Thus, fibromyalgia is diagnosed if the person has: a widespread pain index (WPI) of 7 or greater and a symptom severity scale score of 5 or greater, or a WPI of between 3 and 6 and a symptom severity scale score of 9 or greater; symptoms have persisted at a similar level for three months or greater; and the pain cannot be explained by another disorder.

While some rheumatologists have thought of fibromyalgia as a specific pain disorder, other investigators have characterised it as a bodily distress syndrome or a physical symptom disorder, or somatoform disorder (Wolfe 2014). It is a heterogeneous condition in which there is abnormal processing of the sensation of pain. The cause, or causes, are not well understood, but it has features in common with neuropathic pain, including changes in the central nervous system (CNS). Moreover, people with neuropathic pain and some people with fibromyalgia experience similar sensory phenomena (Koroschetz 2011). Many people with fibromyalgia are significantly disabled, and experience moderate or severe pain for many years. Chronic painful conditions comprised five of the 11 top-ranking conditions for years lived with disability in 2010 (Vos 2012), and are responsible for considerable loss of quality of life and employment, and increased health costs (Moore 2014a).

Fibromyalgia is common. Numerous studies have investigated prevalence in different settings and countries. The review by Queiroz 2013 gave a global mean prevalence of 2.7\% (range $0.4 \%$ to $9.3 \%$ ), and a mean in the Americas of $3.1 \%$, in Europe of $2.5 \%$, and in Asia of $1.7 \%$. Fibromyalgia is more common in women, with a female to male ratio of $3: 1$ (4.2\%:1.4\%). The change in diagnostic criteria does not appear to have significantly affected estimates of prevalence (Wolfe 2013). Estimates of prevalence in specific populations vary greatly, but have been reported to be as high as $9 \%$ in female textile workers in Turkey and $10 \%$ in metalworkers in Brazil (59\% in those with repetitive strain injury; Queiroz 2013). Risk factors for fibromyalgia include: sex (it is more common in women than in men); family history (it is more likely if a relative has the condition); age (it is more common as age increases); and rheumatic disease (rheumatoid arthritis or lupus) (Wolfe 2013). The financial burden of fibromyalgia on society is significant. One cross-sectional study on 299 people with fibromyalgia in France and Germany estimated that, on average, people visited their physician 11.6 (France) and 19.6 (Germany) times per year and missed 32.4 (France) and 25.2 (Germany) days of work per year (Winklemann 2011). Total annual costs to society based on three-month data from 2008 were EUR 7900 in France and EUR 7256 in Germany per person. Direct costs from physician clinic visits, medications, and out-of-pocket expenses were EUR 910 (France) and EUR 1765 (Germany), and indirect costs from missed days of work and lost productivity were EUR 6990 (France) and EUR 5491 (Germany).

There are no definitive treatments for fibromyalgia. Fibromyalgia pain is difficult to treat effectively, with only a minority of people experiencing a clinically relevant benefit from any one intervention. A multidisciplinary approach is now advocated, with pharmacological interventions being combined with physical or cognitive interventions, or both. Conventional analgesics are usually not effective. Treatment is often by so-called unconventional analgesics, such as antidepressants such as duloxetine and amitriptyline (Lunn 2014; Moore 2012a; Sultan 2008), or antiepileptic drugs such as gabapentin or pregabalin (Moore 2009; Moore 2011a; Wiffen 2013). The proportion of people who achieve worthwhile pain relief (typically at least a 50\% reduction in pain intensity; Moore 2013a) is small, generally only $10 \%$ to $25 \%$ more than with placebo, with numbers needed to treat for an additional beneficial outcome (NNTB) usually between four and 10 (Wiffen 2013). People who do experience good levels of pain relief, however, also benefit from substantial reductions in other symptoms, such as fatigue, function, sleep, depression, anxiety, and ability to work, with significant improvement in quality of life (Moore 2010b; Moore 2014a; Straube 2011). Fibromyalgia is not particularly different from other chronic pain in that only a small proportion of study participants have a good response to treatment (Moore 2013a).

\section{Description of the intervention}

Transcutaneous electrical nerve stimulation (TENS) is the delivery of pulsed electrical currents across the intact surface of the skin to stimulate peripheral nerves, principally for pain relief (APTA 2001; Johnson 2014). TENS treatment is usually self-administered, ideally following instruction from a healthcare practitioner. A portable, battery-powered TENS device is used to produce the electrical currents and these are delivered to the body using self-adhering electrodes attached to the surface of the skin. TENS is inexpensive, with a good safety profile compared with medication. TENS devices and accessories (lead wires and selfadhering electrodes) are available without prescription. In some countries TENS needs to be prescribed by a healthcare practitioner to claim reimbursement from social security or health insurance companies. Professional bodies published robust safety guidelines to guide judgements about the appropriateness of TENS in 
certain situations (Houghton 2010). Contraindications include people who have cardiac pacemakers and implantable cardioverter defibrillators. Precautions include pregnancy, epilepsy, active malignancy, deep-vein thrombosis, and frail or damaged skin (Johnson 2011).

TENS devices create pulsed currents with asymmetrical biphasic rectangular or symmetrical biphasic rectangular waveforms. TENS devices are designed so that users can adjust the electrical characteristics of the currents including: pulse frequency (usually less than $200 \mathrm{~Hz}$ ), pulse amplitude (usually less than $70 \mathrm{~mA}$ ), pulse duration (usually $50 \mu$ seconds to $250 \mu$ seconds), and pulse pattern (sometimes termed 'mode' and including continuous, burst, and modulated). Modulated pulse patterns may help to reduce tolerance to TENS caused by repeated use and include modulated frequency, modulated amplitude, and modulated duration (Sluka 2013).

The International Association for the Study of Pain defined two TENS techniques which are commonly used in the literature (Charlton 2005): conventional TENS administered using highfrequency, low-intensity currents to produce a strong non-painful TENS sensation; and acupuncture-like TENS (AL-TENS) using lowfrequency, high-intensity currents to produce strong non-painful pulsate sensations, phasic muscle contractions (twitching), or both (Claydon 2008a). Low-frequency TENS is consistently defined as the delivery of pulsed current of $10 \mathrm{~Hz}$ or less or low-frequency trains (bursts) of high-frequency pulsed current (i.e. burst mode TENS). High-frequency TENS is often described as pulsed current between about $50 \mathrm{~Hz}$ and $100 \mathrm{~Hz}$, although this neglects frequencies between $11 \mathrm{~Hz}$ and $49 \mathrm{~Hz}$ and frequencies above $100 \mathrm{~Hz}$. The term medium-frequency TENS is rarely used in the literature so high-frequency TENS should be used to describe frequencies greater than $10 \mathrm{~Hz}$ to the maximum setting on the TENS device, which is usually $150 \mathrm{~Hz}$ to $200 \mathrm{~Hz}$ (Johnson 2014). High-frequency TENS is not always applied at a low intensity and low-frequency TENS is not always applied at a high intensity. Low-frequency TENS applied $10 \%$ below motor threshold generates analgesia in humans and reduces primary and secondary joint inflammation in animal models of nociception (Chen 2008; King 2001; Sluka 1998; Sluka 2013; Vance 2007). The critical factor for response to TENS is the perceptual experience of the intensity of currents during stimulation regardless of frequency. Evidence suggests that optimal hypoalgesia is achieved using pulse amplitudes $(\mathrm{mA})$ that generate a strong, non-painful TENS sensation and therefore pulse amplitude should be titrated during treatment to maintain this intensity level (Bjordal 2003; Moran 2011; Sluka 2013). Thus, we intended to undertake a subgroup analysis of intensity ('strong' versus 'barely perceptible'), frequency (low frequency versus high frequency when intensity is 'strong'), and technique (conventional TENS versus AL-TENS), if sufficient data were available.

Response to TENS is also influenced by site of stimulation according to the placement of electrodes. Best practice guidelines suggest that electrodes should be placed on healthy sensate skin so that the TENS sensation covers (permeates) the painful area. This is achieved by placing electrodes directly over or 'bracketing' the painful site. This may not always be possible because, for example, skin sensation is altered, there is a skin lesion, or a body part is absent. In these circumstances, electrodes are placed over the main nerves proximal to the site of pain, close to vertebrae of spinal segments, over contralateral dermatomes, over acupuncture points (acu-TENS), or over myofascial trigger points. Research findings on the effect of the site of stimulation on treatment outcome are ambiguous (Johnson 2014). Consideration also needs to be given to the duration and regularity of treatment and the timing of outcome measurements. In particular, evidence suggests that the effects of TENS are maximal during stimulation or immediately after stimulation (Sluka 2013), and that some studies have failed to measure outcome during stimulation (Bennett 2011; Bjordal 2003). Thus, we intended to undertake a subgroup analysis of during TENS versus after TENS if sufficient data were available.

\section{How the intervention might work}

The theoretical underpinning for pain relief by electrical stimulation of the skin was established through the publication of the Gate Control Theory of Pain by Melzack and Wall (Melzack 1965). They proposed that neural activity in low-threshold cutaneous afferents (e.g. A-beta axons) would inhibit onward transmission of nociceptive (pain-related) information in the spinal cord and brainstem. Normally, activity in low-threshold cutaneous afferents is generated by low-intensity mechanical stimuli such as 'rubbing the skin.' They suggested that electrical currents could be used to stimulate the low-threshold cutaneous afferents to reduce pain. The physiological intention of using conventional TENS is to generate a strong but non-painful TENS sensation as this is indicative of selective activation of low-threshold cutaneous afferents (A-beta axons). Evidence suggests that this inhibits onward transmission of nociceptive information at the first synapse in the spinal cord or brain stem (i.e. segmental modulation; Garrison 1996; Ma 2001). The intention of using AL-TENS is to generate pulsate sensations in the skin and underlying tissue or non-painful muscle twitching (or both) as this produces neural activity in small diameter muscle afferents leading to activation of descending pain inhibitory pathways (DeSantana 2009; Francis 2011; Kalra 2001; Millan 2002). TENS may also reduce nociceptive input to the CNS by blocking incoming afferent activity in peripheral neurons, creating a 'busy-line' effect (Nardone 1989).

Research on animals suggests that low-frequency TENS, when administered just below motor threshold, mediates effects via noradrenaline, serotonin, and $\mu$-opioid systems and highfrequency TENS, when administered just below motor threshold, mediates effects via noradrenaline, gamma-aminobutyric acid (GABA), and $\delta$-opioid systems (Kalra 2001; Leonard 2010; Maeda 2007; Santos 2013; Sluka 1999; Sluka 2006; Somers 2009). Whether the frequency-mediated effects of TENS translate into differential hypoalgesia in humans when the intensity of TENS is kept constant remains in doubt (Chen 2008; Claydon 2008a). There is evidence that long-term use of opioid medication may impact negatively on response to low-frequency TENS but not on response to highfrequency TENS (Sluka 2000). Leonard 2011 found that highfrequency TENS reduced pain in 12 opioid-treated people with chronic pain and 11 opioid-naïve people with chronic pain, whereas low-frequency TENS only reduced pain in the non-opioid group. The lack of pain relief during low-frequency TENS was attributed to the development of $\mu$-opioid receptor tolerance.

\section{Sham credibility issues in studies of transcutaneous electrical nerve stimulation}

Bennett 2011 examined aspects of fidelity that may contribute to a risk of bias in TENS studies. Factors that contributed to the overestimation of TENS effects included inadequate method of 
randomisation, small sample sizes, and issues associated with the implementation of a sham (placebo) control such as allocation concealment and how blinding was maintained. Various types of sham control have been used in TENS studies, including deactivated TENS devices that are identical in appearance but deliver no current and TENS devices that deliver stimulation at the start of treatment and fade to zero current output over a brief period of time (e.g. within 45 seconds) (Rakel 2010). There are threats to the credibility of this approach because active stimulation elicits sensations and introduces a risk of bias to sham-controlled interventions. Thus, it is not possible to truly blind the person to the sensory experience generated by different types of TENS or the lack of sensation during sham (no current) TENS (or both). However, the nature of the TENS intervention can be concealed during pre-study briefing using a process that calibrates the participant's expectations of sensations from study interventions. Participants can be briefed that some types of non-invasive electrical stimulation techniques do not produce sensations during stimulation (i.e. microcurrent therapy) and that they may or may not experience sensations from the TENS device (Bennett 2011). The sham (no current) device can look and behave similarly to the intervention device (e.g. identical appearance of the device, flashing lights, and functioning display panel) and participants can be instructed to use the device at a predetermined setting on the display. Blinding can be monitored by asking participants whether they believed that "...the device was functioning properly?" (Deyo 1990). Bennett 2011 also examined aspects of fidelity that may contribute to underestimation of the effects of TENS and found that the adequacy of the TENS intervention (i.e. the appropriateness of the TENS technique) was the main area of concern. Other factors contributing to underestimation of the effects of TENS were: a lack of instruction on how best to administer TENS especially when self-administering TENS; assessment of adherence; inadequate reporting of the TENS regimen during use; and failure to standardise or report concurrent analgesia and to assess comparability between groups. We intended to undertake a subgroup analysis of TENS administered as a sole treatment versus TENS administered in combination with other treatments if sufficient data were available.

\section{Why it is important to do this review}

TENS is used extensively to manage painful conditions because it has few contraindications or reported adverse effects and has no potential for overdose (Johnson 2014). One Cochrane Review by Johnson 2015a concluded that there was tentative evidence that TENS reduces pain intensity when administered as a stand-alone treatment for acute pain in adults and a nonCochrane meta-analysis found superiority of TENS over placebo for reducing postoperative analgesic consumption (Bjordal 2003). Another Cochrane Review found only limited evidence of effect for labour pain (Dowswell 2009). In 2008, one Cochrane Review on TENS for chronic pain was inconclusive (Nnoaham 2008); although the 2008 review has now been withdrawn, our new review will partly serve to update it, focusing on fibromyalgia alone. Most Cochrane Reviews on specific chronic pain conditions have found the evidence to be inconclusive (e.g. osteoarthritis of the knee (Rutjes 2009)) or insufficient to make a judgement (e.g. chronic low back pain (Khadilkar 2008), cancer pain (Hurlow 2012), and phantom pain and stump pain (Johnson 2015b)). Non-Cochrane meta-analyses have found superiority of TENS over placebo for chronic musculoskeletal pain (Johnson 2007), and osteoarthritis of the knee (Bjordal 2007). Often systematic reviews and metaanalyses are hindered by methodological weaknesses including suboptimal TENS interventions and inadequate study sample sizes (Bennett 2011: Johnson 2010; Sluka 2013). One overview of Cochrane Reviews of TENS for chronic pain is in development (Catley 2015), and a new review on TENS for neuropathic pain in adults, which was developed from Claydon 2010, has been published (Gibson 2017).

There is evidence that electrode placement site, frequency, and intensity of stimulation influence TENS outcome although the precise nature of the interaction is unknown (Chesterton 2003; Claydon 2008b; Claydon 2013). Studies on healthy people exposed to experimental pain and systematic reviews with meta-analyses of people with painful conditions have found that a strong, nonpainful TENS sensation, at or close to the site of pain, produces optimal analgesic efficacy (Aarskog 2007; Bjordal 2007; Chen 2011; Claydon 2008a; Moran 2011). Central sensitisation contributes to pain associated with fibromyalgia and therefore TENS may be beneficial because it has been shown to reduce this (Ma 2001).

Exercise is recommended as a treatment for fibromyalgia (Macfarlane 2017) and evidence suggests that aerobic exercise may slightly decrease pain intensity (Bidonde 2017), although adherence to exercise programmes may be poor due to pain and fatigue (Bidonde 2014; Busch 2008). As TENS reduces pain during movement, it may be useful as an adjunct to assist with participation in exercise and activities of daily living. Some clinical studies have been published on TENS for managing symptoms associated with fibromyalgia (Dailey 2013; Lauretti 2013; Mutlu 2013), and there is a published protocol for a double-blind randomised clinical study that has yet to be completed (Noehren 2015). Two large questionnaire surveys on people with fibromyalgia revealed that $21 \%$ of people in the US (Bennett 2007) and $17 \%$ of Germans with fibromyalgia (Häuser 2012) reported use of TENS for symptom control. To date, only one systematic review exists which looks at physiotherapy and physical agents used for fibromyalgia, of which TENS is included (Winkelmann 2012). Three randomised controlled trials (RCTs) with 82 participants and mean study duration of five (range three to five) weeks were analysed but studies were found to be of low methodological quality and incompletely reported. The authors concluded that TENS was not an effective treatment option for fibromyalgia. An updated systematic review of the current evidence of the effects of TENS for fibromyalgia is needed so that health professionals, researchers, and people with fibromyalgia can make informed decisions about its use.

The standards used to assess evidence in chronic pain studies have changed substantially, with particular attention being paid to study duration, withdrawals, and statistical imputation following withdrawal, all of which can substantially alter estimates of efficacy. The most important change is the move from using mean pain scores, or mean change in pain scores, to the number of people who have a large decrease in pain (by at least 50\%) and who continue in treatment, ideally in studies of eight to 12 weeks or longer. Pain intensity reduction of $50 \%$ or more has been shown to correlate with improvements in comorbid symptoms, function, and quality of life. These standards are set out in the reference guide for pain studies (PaPaS 2012). This Cochrane Review assessed evidence using methods that make both statistical and clinical sense, and will use developing criteria for what constitutes reliable 
evidence in chronic pain (Moore 2010a). The studies included and analysed needed to meet a minimum of reporting quality (blinding, randomisation), validity (duration, dose and timing, diagnosis, outcomes, etc.), and size (ideally at least 500 participants in a comparison in which the NNTB is four or above; Moore 1998). This approach sets high standards and marks a departure from how reviews were conducted previously.

\section{O B J E C T I VES}

To assess the analgesic efficacy and adverse events of TENS alone or added to usual care (including exercise) compared with placebo (sham) TENS; no treatment; exercise alone; or other treatment including medication, electroacupuncture, warmth therapy, or hydrotherapy for fibromyalgia in adults.

\section{METHODS}

\section{Criteria for considering studies for this review}

\section{Types of studies}

We included RCTs or quasi-randomised trials of TENS treatment. We included single treatment interventions without follow-up and gave credence to studies that delivered at least two weeks of treatment and had a study duration of at least eight weeks. We included cross-over and parallel-group study designs. We required full journal publication, with the exception of online clinical study results summaries of otherwise unpublished clinical studies, and abstracts with sufficient data for analysis. We did not include short abstracts (usually meeting reports). We excluded studies that were non-randomised, studies of experimental pain, case reports, and clinical observations.

\section{Types of participants}

We included studies of adults aged 18 years or above with pain due to fibromyalgia diagnosed using either the Wolfe (Wolfe 1990; Wolfe 2010) or earlier criteria in the case of older studies (e.g. Goldenberg 1987).

\section{Types of interventions}

We included studies that evaluated TENS administered using non-invasive techniques for pain relief. We excluded invasive techniques such as percutaneous electrical nerve stimulation. We included TENS administered using a standard TENS device (Johnson 2014), regardless of the device manufacturer, which delivered biphasic or monophasic pulsed electrical currents that were greater than $1 \mathrm{~mA}$ using at least two surface electrodes. We excluded TENS delivered using single probe electrodes (i.e. TENS pens) and studies investigating 'TENS-like' devices such as neuromuscular electrical stimulation (NMES) devices and interferential current devices. We included studies that administered TENS at intensities that produced perceptible TENS sensations during stimulation. To explore suboptimal stimulation, we conducted a subgroup analysis to compare TENS at intensities described as 'strong' (optimal) versus those described as 'barely perceptible,' 'faint,' or 'mild' (suboptimal). We included TENS administered on an area of the body that was sensate at either the site of pain or over nerve bundles proximal (or near) to the site of pain. We included AL-TENS delivered at strong intensities to generate muscle twitches. We only included TENS delivered at acupuncture points if the point was lying over nerve bundles proximal (or near) to the site of pain. We included any TENS parameters meeting these criteria; any duration or regularity of TENS treatment; and either self-applied or therapist-applied TENS treatment. We included TENS administered as a sole treatment or in combination with usual care. We included studies that evaluated TENS versus:

- placebo TENS (e.g. sham (no current) TENS device);

- no treatment or waiting list control;

- usual care (including exercise);

- other treatment.

Sham credibility is an issue in TENS studies (Deyo 1990). We defined a sham TENS device as a device similar to the one used in the active group but where the current output was modified so that there was: no electrical current, a barely perceptible electrical current, or electrical current that ceased within one minute (Rakel 2010; Sluka 2013). We excluded studies where it was not possible to isolate the effects of TENS from other treatments.

\section{Types of outcome measures}

We anticipated that studies would use a variety of outcome measures, with the majority of studies using standard subjective scales (numerical rating scale (NRS) or visual analogue scale (VAS)) for pain intensity or pain relief, or both. We included measures of pain at rest and pain on movement. We were particularly interested in Initiative on Methods, Measurement, and Pain Assessment in Clinical Trials (IMMPACT) definitions for moderate and substantial benefit in chronic pain studies (Dworkin 2008). These were defined as:

- at least $30 \%$ pain relief over baseline (moderate);

- at least 50\% pain relief over baseline (substantial);

- much or very much improved on Patient Global Impression of Change scale (PGIC; moderate);

- very much improved on PGIC (substantial).

These outcomes concentrate on dichotomous outcomes where pain responses do not follow a normal (Gaussian) distribution. People with chronic pain desire high levels of pain relief, ideally more than $50 \%$, and ideally with no worse than mild pain (Moore 2013b; O'Brien 2010). We included 'Summary of findings' tables as set out in the author guide (PaPaS 2012) where sufficient data were available. The 'Summary of findings' table included outcomes of at least $30 \%$ and at least $50 \%$ pain intensity reduction, PGIC, withdrawals due to adverse events, serious adverse events, and death. We used the GRADE approach to assess the quality of evidence related to each of the key outcomes listed in Types of outcome measures (Chapter 12, Higgins 2011), as appropriate. We planned to extract outcome measurement data before, during, and after the intervention, where data were available.

\section{Primary outcomes}

- Participant-reported pain relief of $30 \%$ or greater, compared with baseline.

- Participant-reported pain relief of $50 \%$ or greater, compared with baseline.

- PGIC much or very much improved.

- PGIC very much improved. 


\section{Secondary outcomes}

- Any pain-related outcome indicating some improvement (e.g. outcomes from continuous data such as participantreported change in pain intensity reported as mean data. We intended to make generalised statements if baseline status was heterogeneous, and large effects in some participants were masked by small effects in others).

- Any participant-reported change in health-related quality of life, including activities of daily living and fatigue, using any validated tool (e.g. 36-item Short Form (SF-36), 6-item Short Form (SF-6), Euroqol).

- Withdrawals due to lack of efficacy, adverse events, and for any cause.

- Participants who experienced any adverse event.

- Participants who experienced any serious adverse event. Serious adverse events typically included any untoward medical occurrence or effect that at any dose resulted in death, was lifethreatening, required hospitalisation or prolongation of existing hospitalisation, resulted in persistent or significant disability or incapacity, was a congenital anomaly or birth defect, was an 'important medical event' that may have jeopardised the person, or may have required an intervention to prevent one of the above characteristics or consequences.

- Specific adverse events, particularly skin reactions, somnolence, and dizziness.

- Any disability-related or mental health-related outcome.

\section{Search methods for identification of studies}

\section{Electronic searches}

We searched the following electronic databases using a combination of controlled vocabulary (i.e. MeSH and free-text terms) to identify published articles:

- Cochrane Central Register of Controlled Trials (CENTRAL; 2016 Issue 12) via CRSO searched on 18 January 2017;

- MEDLINE (via Ovid) from 1950 to 18 January 2017;

- Embase (via Ovid) from 1980 to 18 January 2017;

- CINAHL (via EBSCO) from 1982 to 18 January 2017;

- PsycINFO (via Ovid) from 1806 to 18 January 2017;

- LILACS (via Birme) from 1982 to 18 January 2017;

- PEDRO from 1929 to 18 January 2017;

- Web of Science (SCI, SSCI, CPCI, SCPCI-SSH) to 18 January 2017;

- AMED (via Ovid) from 1985 to 18 January 2017;

- SPORTDiscus (via EBSCO) from 1975 to 18 January 2017.

There were no language restrictions. We tailored the searches to the individual databases. We adapted the MEDLINE search strategy for the other databases listed. The search strategy combined the subject-specific search with phase one and two of the Cochrane Sensitive Search Strategy for RCTs (as published in Sections 6.3.2.1, 6.3.3.2, and 6.4.11.1 in the Cochrane Handbook for Systematic Reviews of Interventions (Higgins 2011)). The search strategies can be found in Appendix 2. We identified all relevant studies irrespective of language and translated articles when possible.

\section{Searching other resources}

We reviewed the bibliographies of RCTs and review articles, and searched clinical trial databases (e.g. ClinicalTrials.gov (www.clinicaltrials.gov), World Health Organization (WHO) International Clinical Trials Registry Platform (ICTRP, apps.who.int/ trialsearch/), and metaRegister of Controlled Trials (mRCT, www.isrctn.com/page/mrct) to identify additional published or unpublished data. We did not contact investigators or study sponsors.

\section{Data collection and analysis}

\section{Selection of studies}

We determined eligibility of studies for inclusion first by title, then by reading the abstract of each record identified by the search. We eliminated records where it was clear from the abstract that they did not satisfy the inclusion criteria, then obtained full copies of the remaining records. Two review authors (MIJ, CAP) made the decisions by reading these records independently and reached agreement by discussion. Disagreements at any stage of the process were/would have been resolved by consensus using a third review author as arbiter (GJ). We did not anonymise the records of studies in any way before assessment. We created a PRISMA flow chart (Higgins 2011; Liberati 2009).

\section{Data extraction and management}

Two review authors (LSC, CAP) extracted data of included studies independently using a standard form and checked for agreement before entry into Review Manager 5 (RevMan 2014). Disagreements were resolved by consensus using the arbiter (MIJ). We included information about:

- country of origin;

- study design: cross-over, parallel-group;

- study duration;

- study participants: age, gender, fibromyalgia diagnostic criteria used, duration of pain and symptoms;

- sample size: active and comparator groups;

- concomitant treatments: pharmacological and nonpharmacological;

- TENS intervention(s) used: type, electrical parameters, electrode location, perceptual experience during intervention including intensity of stimulation, dosing regimen;

- comparison group(s) used: placebo, no treatment, usual treatment, other treatment, dosing regimen;

- outcomes: time points used including follow-up, withdrawals;

- adverse and serious adverse effects;

- other: sponsorship, country of origin, conflict of interest statements.

We used these data to populate the Characteristics of included studies table.

\section{Assessment of risk of bias in included studies}

Two review authors (LSC, CAP) independently assessed risk of bias for each trial, using the criteria outlined in the Cochrane Handbook for Systematic Reviews of Interventions (Higgins 2011), with disagreements resolved by consensus with a third review author (MIJ) acing as arbiter. We assessed the following for each trial.

- Random sequence generation (selection bias). We assessed the method used to generate the allocation sequence as: low 
risk of bias (any truly random process, e.g. random number table; computer random number generator); unclear risk of bias (method used to generate sequence not clearly stated). We excluded studies using a non-random process (e.g. odd or even date of birth; hospital or clinic record number).

- Allocation concealment (selection bias). The method used to conceal allocation to interventions prior to assignment determines whether the intervention allocation could have been foreseen in advance of, or during, recruitment or changed after assignment. We assessed the methods as: low risk of bias (e.g. telephone or central randomisation; consecutively numbered, sealed, opaque envelopes); unclear risk of bias (method not clearly stated); high risk of bias (studies that did not conceal allocation (e.g. open list).

- Blinding of participants and personnel (performance bias): participants, and care providers administering TENS.

- Blinding of participants: low risk of bias (participants blinded to allocated intervention and unlikely that blinding broken); unclear risk of bias (insufficient information to permit judgement of low/high risk of bias); high risk of bias (participants not blinded to allocated intervention OR participants blinded to allocated intervention but it was likely that blinding may have been broken).

- Blinding of personnel (care provider(s) administering TENS): low risk of bias (care provider blinded to allocated intervention and unlikely that blinding broken); unclear risk of bias (insufficient information to permit judgement of low/ high risk of bias); high risk of bias (care provider not blinded to allocated intervention and the two interventions clearly identifiable to the care provider as experimental and control OR care provider blinded to allocated intervention but likely that blinding was broken).

- Blinding of outcome assessment (detection bias): outcome assessors.

* Blinding of assessor: low risk of bias (outcome assessor (including 'participants' with respect to self-report outcomes) blinded to participants' allocated intervention and unlikely that blinding broken); unclear risk of bias (insufficient information to permit judgement of low/high risk of bias); high risk of bias (outcome assessor (including 'participants' with respect to self-report outcomes) unblinded to participants' allocated intervention OR outcome assessor blinded to allocated intervention but likely that blinding was broken)).

- Incomplete outcome data (dropouts). We checked for possible attrition bias by considering if participant dropout was acceptable and described: low risk of bias (less than $20 \%$ dropout and appeared to be random with numbers per group provided along with reasons for dropout); unclear risk of bias (less than 20\% and unclear if random with numbers per group and reasons for dropout not described); high risk of bias (greater than $20 \%$ dropout).

- Incomplete outcome data (protocol violations). We considered if participants were analysed as per original group allocation: low risk of bias (if participants were analysed in the group to which they were originally assigned); unclear risk of bias (where insufficient information was provided to determine if analysis was per protocol or intention-to-treat (ITT)); high risk of bias (where per protocol analysis was used, where available data were not analysed or participants' data were included in the group to which they were not originally assigned).

- Selective reporting. We assessed whether studies selectively reported outcomes. We assessed the methods as: low risk of bias (study protocol was available and all prespecified outcomes were reported or study protocol was not available but all expected outcomes were reported); unclear risk of bias (inadequate information to allow judgement of a study to be classified as 'low risk' or 'high risk'); high risk of bias (incomplete reporting of specified outcomes. One or more primary outcomes were reported using measurements or analysis that were not prespecified. One or more of the primary outcomes was not prespecified. One or more outcomes of interest were reported incompletely and could not be entered into a meta-analysis. Results for a key outcome expected to be reported were excluded).

- Size of study (checking for biases confounded by small size). We assessed this as: low risk of bias (200 participants or more per treatment arm); unclear risk of bias (50 to 199 participants per treatment arm); high risk of bias (fewer than 50 participants per treatment arm).

- Other sources of bias. We considered other factors such as whether studies were stopped early, there were differences between groups at baseline, the timing of outcome measurement, cointervention comparability, and funding declarations.

\section{Measures of treatment effect}

Where available and appropriate we presented quantitative and ITT data. For dichotomous data (responder analyses), we used the Initiative on Methods, Measurement and Pain Assessment in Clinical Trials (IMMPACT) definitions for interpreting the clinical importance in change in outcome measures compared with baseline (Dworkin 2008). We intended to calculate risk ratio (RR) and risk difference (RD) with 95\% confidence intervals $(\mathrm{Cl})$ for dichotomised outcome measures. We intended to calculate the NNTB as an absolute measure of treatment effect where possible. We intended to present pain outcomes collected as continuous data on identical scales as mean difference (MD) with $95 \% \mathrm{Cl}$. We intended to present pain outcomes collected as continuous data using different scales as standardised mean difference (SMD) with $95 \% \mathrm{Cl}$. We intended to interpret reductions in pain over baseline as follows:

- less than $15 \%$ : no important change;

- $15 \%$ or greater: minimally important change;

- $30 \%$ or greater: moderately important change;

- $50 \%$ or greater: substantially important change.

For health-related quality of life data, we intended to consider a clinical difference greater than $10 \%$ of the scale employed to be minimally important (Furlan 2009).

The IMMPACT thresholds are based on estimates of the degree of within-person change from baseline that participants might consider to be clinically important. We expected that the studies in this review would most likely present effect sizes as the mean between-group change between intervention groups. There is little consensus or evidence regarding what the threshold should be for a clinically important difference in pain intensity based on the between-group difference during or after the intervention. It has 
been found that in pharmacological studies, pain outcomes for acute pain (Moore 2011b; Moore 2011c), back pain (Moore 2010d), arthritis (Moore 2010c), and fibromyalgia (Straube 2010), tend to have a U-shaped rather than a bell-shaped distribution, with some people experiencing a substantial reduction in symptoms, some minimal to no improvement, and few experiencing mean (moderate) improvement. Thus, data expressed as means may be misleading as a small mean between-group effect size may represent a proportion of participants that actually responded very well to the intervention (Moore 2013c; Moore 2014a). It is unknown whether outcomes are commonly bimodally distributed in studies of TENS. The advantage of focusing on the betweengroup difference is that it is the only direct estimate of the mean specific effect of the intervention and a small mean betweengroup effect might accurately represent very small effects of the intervention for most or all people. We intended to use a threshold of $10 \mathrm{~mm}$ on a 0 - to $100-\mathrm{mm}$ VAS for minimally important outcome for pain when analysing mean between-group change, in line with the OMERACT 12 group, which states that the proportion of people achieving one or more thresholds of improvement from baseline pain (e.g. greater than $10 \%, 20 \%$ or greater, $30 \%$ or greater, $50 \%$ or greater) should be reported in addition to mean change (Busse 2015). We would interpret these findings with caution as it remains possible that estimates that fall close to this point may reflect a treatment that benefits an appreciable number of people.

\section{Unit of analysis issues}

We intended to split the control treatment arm between active treatment arms in a single study if the active treatment arms were not combined for analysis. In the unlikely event that the unit of randomisation is not the participant, or where a cross-over design was used, we intended not to include the data unless a suitable adjustment for the study design had been, or could be, made. We included cross-over designs but intended only to enter the first period data into the meta-analysis. If this was not reported, we intended to note this and not include the data. If data were reported appropriately, then we intended to include the data using the generic inverse variance feature.

\section{Dealing with missing data}

We intended to use ITT analysis where the ITT population consisted of participants who were randomised, received at least one dose of the assigned study intervention, and provided at least one postbaseline assessment. We intended to assign missing participants zero improvement wherever possible.

\section{Assessment of heterogeneity}

We intended to deal with clinical heterogeneity by combining studies that examined similar conditions. We performed separate analyses where TENS was compared with different control conditions such as placebo or no treatment control. We intended to examine heterogeneity using visual inspection of forest plots, the $\mathrm{I}^{2}$ statistic, and L'Abbé Plots (L'Abbé 1987), and the Chi ${ }^{2}$ test, if appropriate. Where significant heterogeneity existed, we intended to explore subgroup analyses. Preplanned comparisons are described in the Subgroup analysis and investigation of heterogeneity section.

\section{Assessment of reporting biases}

The aim of this review was to use dichotomous outcomes of known utility and of value to patients (Moore 2010b; Moore 2010c; Moore 2010d; Moore 2013b). It was intended that the review would not depend on what the authors of the original studies chose to report or not, though clearly difficulties would arise in studies failing to report any dichotomous results. We planned to extract and use continuous data, which would probably poorly reflect efficacy and utility and therefore be useful for illustrative purposes only. We intended to assess publication bias using a method designed to detect the amount of unpublished data with a null effect required to make any result clinically irrelevant (usually taken to mean a NNTB of 10 or higher; Moore 2008). We considered the possible influence of small-study samples by the risk of bias criterion "study size." If at least 10 studies were included in a meta-analysis and included studies differed in sample size, we planned to visually inspect funnel plots to explore the likelihood of reporting biases. For studies that used continuous outcomes, we intended to use Egger's test to detect small-study bias (Higgins 2011). We would have interpreted the results of this process cautiously since we were aware that all approaches to the quantification of possible reporting biases have important limitations (Moore 2008).

\section{Data synthesis}

We intended to perform pooling of results where adequate data existed using Review Manager 5 (RevMan 2014). We would have undertaken meta-analyses of outcome data only from suitably homogeneous studies using a random-effects model. Where possible, we would have grouped extracted data according to outcome and measurement time points. Time points would have included during stimulation or immediately after stimulation at each treatment session, or both; and post-intervention follow-up at less than two weeks' post-intervention (short-term), two to seven weeks' post-intervention (mid-term), and eight weeks or more post-intervention (long-term).

\section{Quality of the evidence}

For all analyses, we intended to explicitly and clearly present the outcome of the 'Risk of bias' assessments in the reporting. Where inadequate data were found to support statistical pooling, we planned to complete a narrative synthesis. Two review authors (MIJ, CAP) independently rated the quality of the outcomes. We used the GRADE (Guyatt 2008) system to rank the quality of the evidence using the GRADEprofiler Guideline Development Tool software (GRADEpro GDT 2015), and the guidelines provided in Chapter 12.2 of the Cochrane Handbook for Systematic Reviews of Interventions (Higgins 2011). The GRADE approach uses five considerations (study limitations, consistency of effect, imprecision, indirectness, and publication bias) to assess the quality of the body of evidence for each outcome. The GRADE system uses the following criteria for assigning grade of evidence:

- high: we are very confident that the true effect lies close to that of the estimate of the effect;

- moderate: we are moderately confident in the effect estimate; the true effect is likely to be close to the estimate of effect, but there is a possibility that it is substantially different;

- low: our confidence in the effect estimate is limited; the true effect may be substantially different from the estimate of the effect; 
- very low: we have very little confidence in the effect estimate; the true effect is likely to be substantially different from the estimate of effect.

We decreased the grade rating by one (-1) or two (-2) if we identified:

- serious (-1) or very serious (-2) limitation to study quality;

- important inconsistency (-1);

- some (-1) or major (-2) uncertainty about directness;

- imprecise or sparse data (-1);

- high probability of reporting bias (-1).

We considered single studies to be both inconsistent and imprecise, unless the sample size was greater than 400 participants for continuous data (i.e. more than 200 participants per treatment arm) and greater than 300 events for dichotomous data. We intended to present pooled effects for all primary outcomes and associated GRADE judgements in 'Summary of findings' tables. In certain circumstances, the overall rating for a particular outcome was adjusted as recommended by GRADE guidelines (Guyatt 2013a). For example, we considered whether there were so few data that the results were highly susceptible to the random play of chance, or if a study used last observation carried forward (LOCF) imputation in circumstances where there were substantial differences in adverse event withdrawals, one would have no confidence in the result and would need to downgrade the quality of the evidence by three levels to very low quality. Also, in circumstances where no data were reported for an outcome, we reported the level of evidence as very low quality (Guyatt 2013b). In other circumstances, we would not downgrade for imprecision if $\mathrm{Cls}$ were wide, if the outcome threshold according to how much harm would be acceptable given a benefit or vice versa.

\section{'Summary of findings' tables}

We included four 'Summary of findings' tables to present the main findings in a transparent and simple tabular format. In particular, we included key information concerning the quality of evidence, the magnitude of effect of the interventions examined, and the sum of available data on the outcomes participant-reported pain relief of $30 \%$ or greater, participant-reported pain relief of $50 \%$ or greater, $\mathrm{PGIC}$ very much improved, PGIC much or very much improved, and withdrawals due to adverse events.

\section{Subgroup analysis and investigation of heterogeneity}

We anticipated too few data for any meaningful subgroup analysis. However, if sufficient data were available, we planned the following analyses: where substantial heterogeneity was found $\left(I^{2}\right.$ greater than $40 \%, P<0.1$ ), we would conduct a subgroup analysis investigating the possible impact of the TENS technique on analgesic efficacy. If appropriate, we would have conducted the following analyses.

- Optimal intensity described as 'strong' or greater versus suboptimal intensity described as 'barely perceptible,' 'faint,' or 'mild.'

- Low-frequency (10 Hz or less) TENS versus other frequency (e.g. greater than $10 \mathrm{~Hz}$ ) TENS.

- Conventional TENS (no visible muscle contraction) versus ALTENS (visible phasic muscle contractions).

- Assessment during TENS versus after TENS.

- TENS administered as a sole treatment versus TENS administered in combination with other treatments.

- TENS administered as a single dose versus repetitive dose.

- Opioid-treated participants versus opioid-naïve participants.

\section{Sensitivity analysis}

We anticipated too few data for any meaningful sensitivity analysis. However, if sufficient data were available, we planned to analyse the effect of excluding studies with high risk of bias and the effect of using a random-effects versus a fixed-effect model.

\section{RES U L T S}

\section{Description of studies}

Results of the search

Searches identified 245 potentially relevant records. After removal of 61 duplicates, we screened the titles and abstracts of 184 records and obtained and read the full texts of 19 records (Figure 1). We included eight studies and found four records that were duplicates (Characteristics of included studies table). An abstract by Vance 2015 was categorised as a secondary report of Dailey 2013 because it presented an analysis of data related to achieving higher-intensity TENS that was not included in the original study report. We excluded six studies after screening the full text report (Characteristics of excluded studies table). We identified one ongoing study (Characteristics of ongoing studies table). 
Figure 1. Study flow diagram.

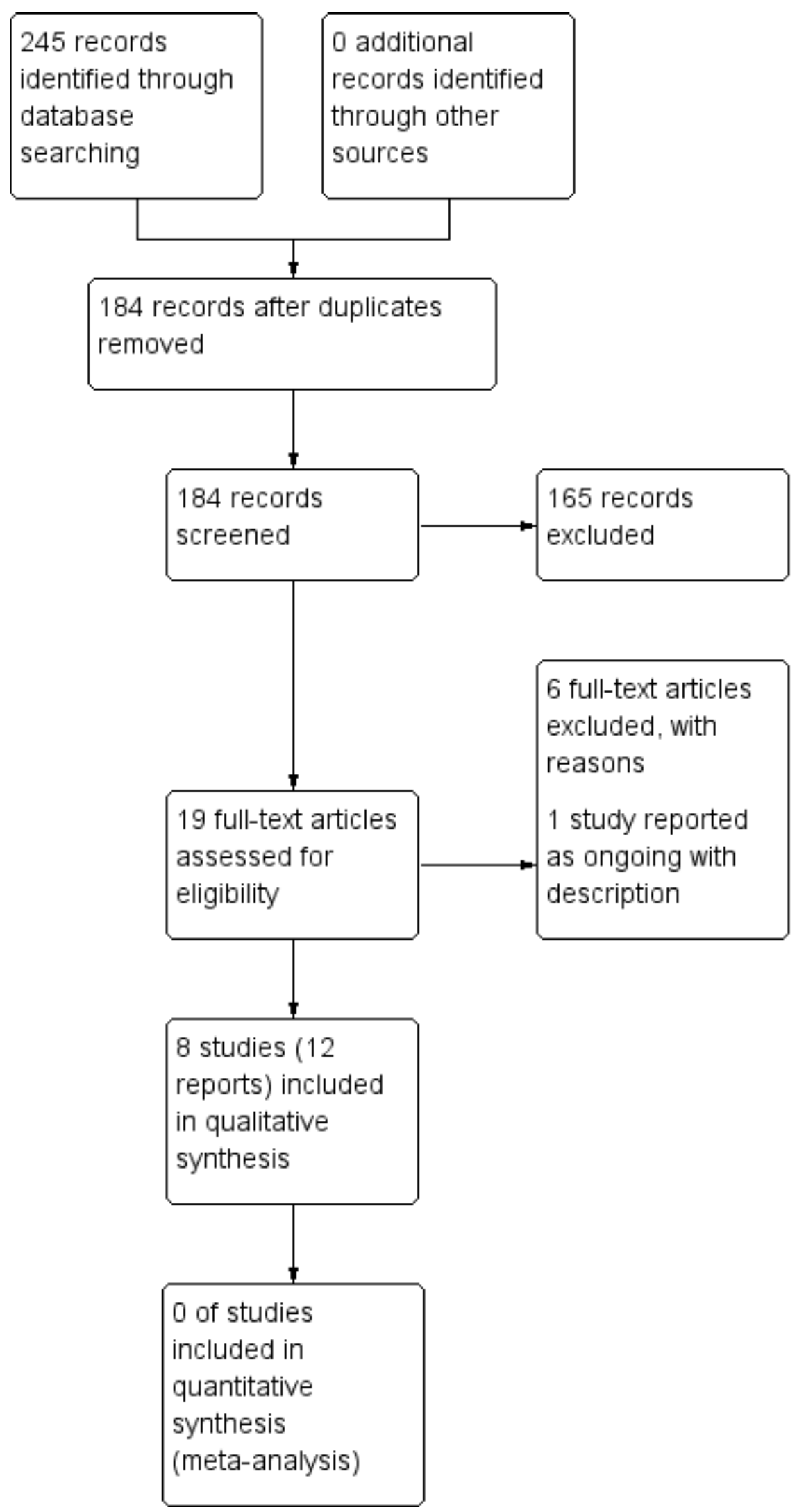




\section{Included studies}

We included eight studies that had 315 adults (299 women) at entry (Carbonario 2013; da Silva 2008; Dailey 2013; Di Benedetto 1993; Guo 2005; Lauretti 2013; Lofgren 2009; Mutlu 2013; see Characteristics of included studies table). The age of participants was 18 to 75 years in the reports that provided information about the age range of the sample. There were seven RCTs and one quasiRCT with sequential allocation to groups (Carbonario 2013). Six studies used a parallel-group design, and two studies used a crossover design (Dailey 2013; Lofgren 2009). Study sample sizes were between 10 and 66 participants and intervention arm size samples were between five and 43 . The period for interventions was 30 minutes (Dailey 2013), seven days (Lauretti 2013), 21 days (da Silva 2008; Lofgren 2009), 42 days (Di Benedetto 1993), and 84 days (although the TENS intervention was only for the first 21 days, Mutlu 2013). The period for interventions used by Carbonario 2013 was unclear. Assessments of treatment outcome were taken within the intervention period. Only one study conducted a post-intervention follow-up, which was for 24 months (Guo 2005).

Six studies used TENS as the sole treatment (da Silva 2008; Dailey 2013; Di Benedetto 1993; Guo 2005; Lauretti 2013; Lofgren 2009), and two studies used TENS in combination with exercise (Carbonario 2013; Mutlu 2013). Five studies used high-frequency TENS (Carbonario 2013; Dailey 2013; Di Benedetto 1993; Lofgren 2009; Mutlu 2013), one study used low-frequency TENS (da Silva 2008), and two studies used both high and low frequencies (Guo 2005; Lauretti 2013). Six studies used 'strong' intensities (Carbonario 2013; Dailey 2013; Guo 2005; Lauretti 2013; Lofgren 2009; Mutlu 2013), one study used 'constant tingling' (Di Benedetto 1993), and one study used 'pleasant tingling' (da Silva 2008). Pain was recorded using VAS (Carbonario 2013; da Silva 2008; Dailey 2013; Lauretti 2013), NRS (Lofgren 2009), or some other pain scale (Di Benedetto 1993; Guo 2005; Mutlu 2013). The most commonly used health-related quality of life was the Fibromyalgia Impact Questionnaire (FIQ) (Carbonario 2013; Lofgren 2009; Mutlu 2013). One study reported outcomes during TENS (Dailey 2013), seven studies reported outcomes post-TENS (Carbonario 2013; da Silva 2008; Di Benedetto 1993; Guo 2005; Lauretti 2013; Lofgren 2009; Mutlu 2013), and two studies reported outcomes long-term from nine weeks (Mutlu 2013) to 24 months (Guo 2005). We provided a brief description of each of the included studies below.

Carbonario 2013 conducted a quasi-randomised controlled parallel-group study that enrolled 32 women sequentially allocated to receive an eight-week programme of aerobic and stretching exercises with or without high-frequency $(150 \mathrm{~Hz})$ TENS applied on bilateral tender points of the trapezium and supraspinatus. Twentyeight women completed the study. Exercises with TENS produced a greater decrease in pain intensity and a greater increase in pressure pain threshold at tender points than exercises without TENS.

da Silva 2008 conducted a parallel-group RCT that enrolled 10 people to receive hydrotherapy or TENS at a frequency of $15 \mathrm{~Hz}$. TENS was administered as three 40-minute sessions per week to a total of 10 sessions. TENS reduced pain, and improved functional outcomes and quality of life to a greater extent than hydrotherapy.

Dailey 2013 conducted a double-blinded randomised, placebocontrolled cross-over study that enrolled 43 women. Each participant received one 30-minute intervention per week of either active TENS at $100 \mathrm{~Hz}$, or placebo TENS or no TENS. The order of interventions was randomised and outcome measures taken whilst TENS remained switched on. Two participants did not receive TENS or placebo TENS because of withdrawal from the study. Active TENS produced higher pressure pain thresholds and lower pain intensity and fatigue with movement compared with placebo and no TENS.

Di Benedetto 1993 conducted a parallel-group RCT that enrolled 30 people to a six-week course of either S-adenosyl-L-methionine (SAMe) or TENS at a frequency of $80 \mathrm{~Hz}$ to $100 \mathrm{~Hz}$. There were decreases in pain, fatigue, and the number of tender points following SAMe but not during TENS.

Guo 2005 conducted a parallel-group RCT that enrolled 66 people to a 20-day course of TENS (dermal neurological electric stimulation), electroacupuncture, or routine medication. TENS was administered daily using two electrodes applied near to the most painful site and two electrodes applied to 'supplementary acupuncture points' at a frequency of $100 \mathrm{~Hz}$ for 15 minutes followed by a frequency of $2 \mathrm{~Hz}$ for 15 minutes. Electroacupuncture was administered using a G-6805 stimulator and No. 28 filiform needles inserted 'deeply' to the same sites as TENS to generate 'deqi.' Routine medication was administered using oral doses of oryzanol and vitamin $B_{1}(30 \mathrm{mg})$, three times a day and amitriptyline titrated to $20 \mathrm{mg}$ to $30 \mathrm{mg}$ once per day. TENS and electroacupuncture reduced pain and recurrence of pain to a greater extent than routine medication.

Lauretti 2013 conducted a randomised placebo-controlled parallelgroup study that enrolled 39 people to one of three interventions: dual-site TENS of the lower back (L5) and the upper back (C7 and T1); dual-site placebo (no current) TENS of the lower back (L5) and the upper back (C7 and T1); or single-site TENS of either the lower back (L5) or upper back (C7 and T1) combined with single-site placebo (no current) TENS of either the lower back (L5) and the upper back ( $\mathrm{C} 7$ and $\mathrm{T} 1$ ). Interventions were administered for 20 minutes per day for seven consecutive days. TENS was administered using mixed frequencies of $2 \mathrm{~Hz}$ and $100 \mathrm{~Hz}$. For the outcomes associated with pain relief and quality of sleep, dual-site TENS was superior to single-site TENS which was superior to dualsite placebo TENS.

Lofgren 2009 conducted a randomised controlled cross-over study that enrolled 32 people to self-administer a daily treatment of superficial warmth $\left(42{ }^{\circ} \mathrm{C}\right)$ using a portable prototype device or TENS at a frequency of $80 \mathrm{~Hz}$ for three weeks with the order of receiving the treatments randomised. Both treatments reduced pain compared with baseline but there were no differences in the amount of pain relief between treatments.

Mutlu 2013 conducted a parallel-group RCT that enrolled 66 people to a 12-week course of supervised exercise with or without TENS at a frequency of $80 \mathrm{~Hz}$. TENS was administered daily in the first three weeks of the 12-week course of exercise. Outcomes for pain, tender point count, FIQ, and SF-36 improved in both groups. There were superior improvements in outcomes for pain at week three for participants receiving TENS.

\section{Excluded studies}

We excluded six records after screening the full-text report (Characteristics of excluded studies table). We excluded one record because we were unable to locate the original source due to an error in the search citation (Mutlu 2006). We excluded one report 
that was a commentary of previously published studies (Simons 2006), one report of a case series (Ido 2003), one report of a study that was found not to be an RCT (Arroyo 1993), one report of a study using microcurrent not TENS (Sunshine 1996), and one report of an RCT where it was not possible to isolate the effects of TENS from other treatment because TENS was given as combination therapy (Kesiktas 2011).

\section{Ongoing studies}

One study was ongoing at the time of this review, for which a protocol had been published (Noehren 2015). The initial phase of the study will randomly allocate 360 participants to receive active TENS, placebo TENS, or standard care (no TENS). See Characteristics of ongoing studies table.

\section{Risk of bias in included studies}

We judged that there was a high risk of bias in seven of the eight included studies. Our assessment of the risk of bias for the included studies is summarised in Figure 2 and Figure 3. We judged the study by Dailey 2013 to have the lowest risk of bias overall, although this study still had a risk of bias due to a small sample size.

Figure 2. Risk of bias graph: review authors' judgements about each risk of bias item presented as percentages across all included studies.

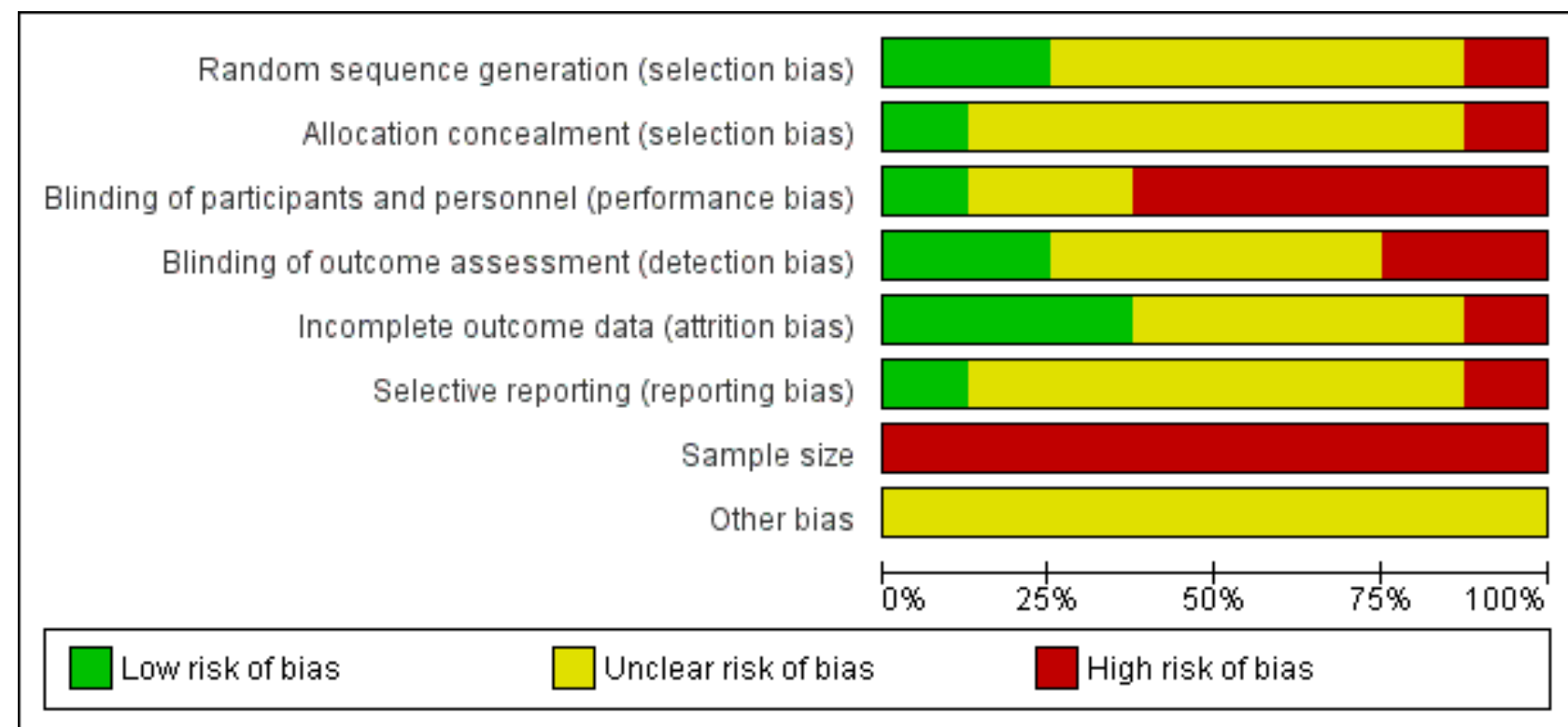


Figure 3. Risk of bias summary: review authors' judgements about each risk of bias item for each included study.

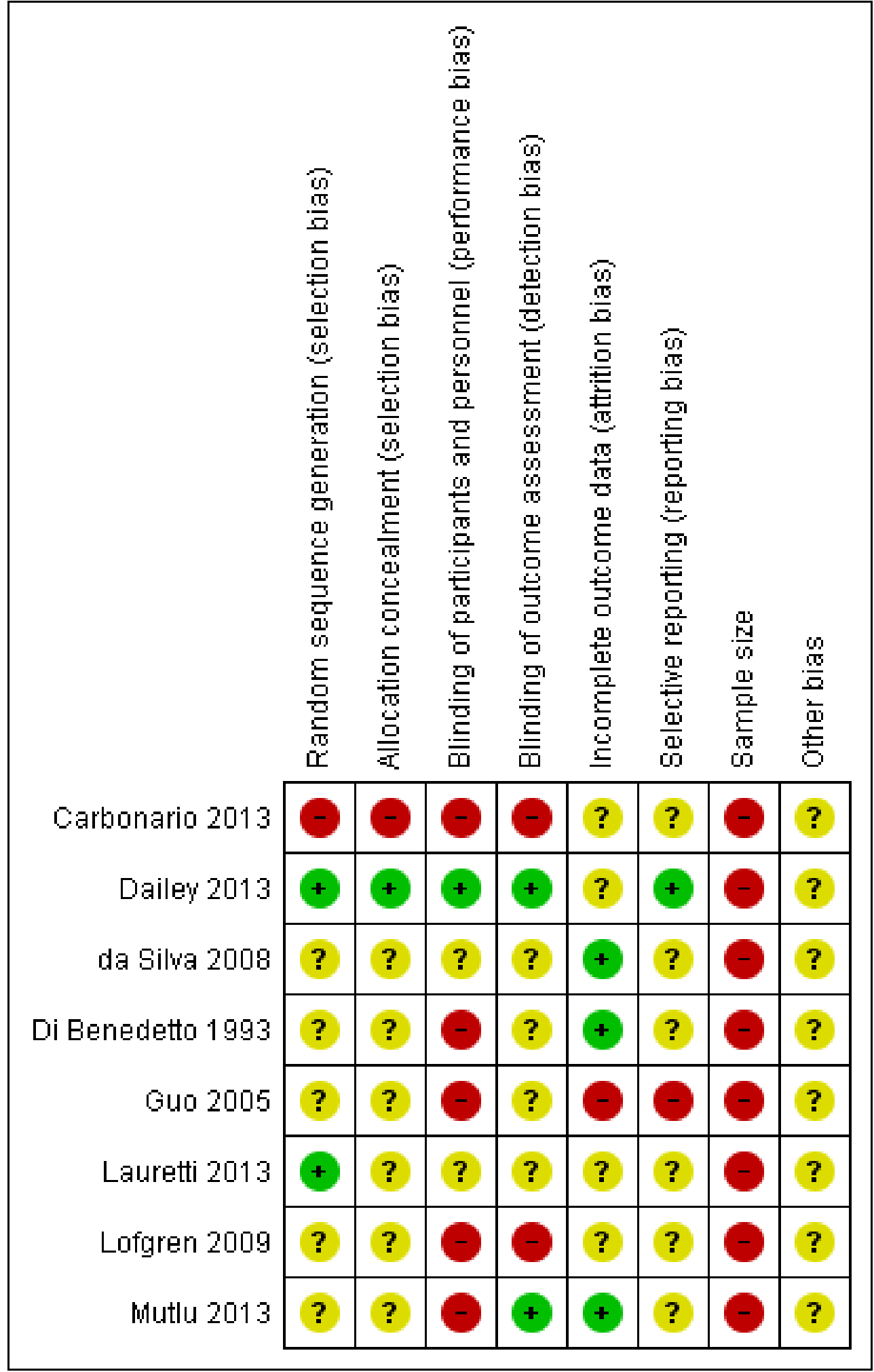

\section{Allocation}

We judged that two studies adequately described the method of random sequence generation (Dailey 2013; Lauretti 2013) and one study adequately described allocation concealment (Dailey
2013). We judged one study to have a high risk of selection bias because participants were allocated consecutively into two treatment groups (Carbonario 2013). We judged the remaining studies to be at unclear risk of bias because they did not adequately 
report the method of random sequence generation and allocation concealment.

\section{Blinding \\ Blinding of participants and personnel (performance bias): participants, and care providers administering TENS}

We judged that two studies adequately described the method of blinding of participants (Dailey 2013; Lauretti 2013) although Lauretti 2013 used TENS with no current and therefore there is a challenge to the success of blinding. Therefore, we judged Dailey 2013 as low risk of bias, and Lauretti 2013 as unclear risk of bias for this domain. We judged five studies to be at high risk of performance bias because there was no attempt to blind participants or therapists (or both) to treatment groups (Carbonario 2013; Di Benedetto 1993; Guo 2005; Lofgren 2009; Mutlu 2013). We judged the remaining study to be at unclear risk of bias.

\section{Blinding of outcome assessment (detection bias): outcome assessors}

We judged that two studies adequately described the method of blinding of outcome assessment (Dailey 2013; Mutlu 2013). Dailey 2013 assessed the success of blinding of outcomes assessors to interventions given simultaneously at two body sites. The success rate of identifying the interventions was 54\% and 53\% for active TENS, $50 \%$ and $34 \%$ for placebo TENS and $58 \%$ and $50 \%$ for no TENS, with no statistically significant differences between treatment groups. We judged two studies to be at high risk of detection bias (Carbonario 2013; Lofgren 2009). We judged the remaining studies to be at unclear risk of bias.

\section{Incomplete outcome data}

We judged three studies to be at low risk of attrition bias because all participants completed the study with no missing outcome data (da Silva 2008; Di Benedetto 1993) or missing outcome data were balanced across the groups with similar reasons for loss (Mutlu 2013). We judged one study to be at high risk of attrition bias because there were substantial data missing at six months', 12 months', and 24 months' follow-up (Guo 2005). We judged the remaining studies to be at unclear risk of attrition bias.

\section{Selective reporting}

We judged one study to be at low risk of reporting bias because there was a protocol registered on ClinicalTrials.gov and analysis of prespecified outcomes were faithfully reported (Dailey 2013). We judged one study to be at high risk of reporting bias because analyses of some outcomes were not prespecified in the methods section and 100-mm VAS scores were converted into four arbitrary categories and analysed using frequency counts (Guo 2005). We judged the remaining studies to be at unclear risk of reporting bias.

\section{Sample size}

We judged that there was a high risk of bias due to small sample sizes with all eight included studies having fewer than 50 participants per treatment arm.

\section{Other potential sources of bias}

We judged that there was a risk of bias from treatment carry-over effects due to the absence of a washout period for Lofgren 2009.
Potential conflicts of interest or industrial sponsorship (or both) were not disclosed in the majority of study reports. Dailey 2013 and Lauretti 2013 disclosed that TENS units were donated by a TENS company. Dailey 2013 disclosed that none of the authors had conflicts of interest with respect to the study although two authors had associations with the company that donated the TENS devices. Di Benedetto 1993 acknowledged partial study support by BioResearch, Liscate, Italy.

\section{Effects of interventions}

See: Summary of findings for the main comparison TENS compared to placebo TENS for fibromyalgia; Summary of findings 2 TENS compared to no treatment for fibromyalgia; Summary of findings 3 TENS with exercise compared to exercise alone for fibromyalgia; Summary of findings 4 TENS compared to other active treatment for fibromyalgia

The authors of seven of the eight included studies concluded that their study provided evidence that TENS was effective at relieving pain associated with fibromyalgia but the studies had very small sample sizes and were underpowered. Small studies are known to be at high risk of producing inaccurate estimates (Moore 1998) and small-study effects distort the findings of meta-analyses (AlBalawi 2013; Nüesch 2010). Stronger effect estimates in meta-analyses are found for small-sized studies compared with larger studies (Dechartres 2013). Thus estimates of efficacy will only be credible if data arise from large studies or from pooling multiple moderately sized studies with similar methodology and comparison groups. The studies in our review did not meet these criteria. We judged that there were too few data from outcomes that were not suitably homogeneous for any meaningful statistical pooling of results. We provide a descriptive narrative of the included studies for completeness (Table 1 ).

\section{TENS compared with placebo TENS (e.g. sham (no current) TENS device)}

Two studies included a comparison of TENS versus placebo TENS (54 participants in the placebo TENS arm (Dailey 2013; Lauretti 2013). We judged the quality of the evidence using GRADE to be very low; we downgraded three times due to a lack of data (Summary of findings for the main comparison).

\section{Primary outcomes}

None of the included studies measured primary outcomes as dichotomous data (responder analysis).

\section{Participant-reported pain relief of $30 \%$ or greater}

Neither study measured participant-reported pain relief of $30 \%$ or greater.

\section{Participant-reported pain relief of $\mathbf{5 0 \%}$ or greater}

Neither study measured participant-reported pain relief of $50 \%$ or greater.

\section{PGIC much or very much improved}

Neither study measured PGIC much or very much improved.

PGIC very much improved

Neither study measured PGIC very much improved. 


\section{Secondary outcomes}

\section{Other pain-related outcomes}

Dailey 2013 found that a single 30-minute treatment of TENS reduced pain intensity (VAS) on movement compared with placebo TENS with the mean \pm standard error of the mean (SEM) difference in VAS scores $(100 \mathrm{~mm})$ before and during intervention being 11.1 $\pm 2.6 \mathrm{~mm}(95 \% \mathrm{Cl} 5.9$ to 16.3$)$ for TENS and $2.3 \pm 2.6 \mathrm{~mm}(95 \% \mathrm{Cl}$ 2.4 to 7.7 ) for placebo TENS. There were no significant differences between TENS and placebo TENS for pain intensity at rest. This double-blinded randomised, placebo-controlled cross-over study of 43 participants was described as a pilot study to inform the design of a larger-scale clinical study. Dailey 2013 reported that active TENS increased pressure pain threshold to a greater extent than placebo TENS in the lumbar region $(P<0.05)$ but not in the cervical region or anterior tibialis.

Lauretti 2013 reported that mean \pm standard deviation (SD) pain intensity (100-mm VAS) decreased from $85 \pm 20 \mathrm{~mm}$ at baseline to $43 \pm 20 \mathrm{~mm}$ after one week of dual-site TENS (reduction 49.4\%) and decreased from $85 \pm 10 \mathrm{~mm}$ at baseline to $60 \pm 10 \mathrm{~mm}$ after one week of single-site TENS (pain reduction 29\%). Placebo TENS decreased pain intensity (VAS) from $82 \pm 20 \mathrm{~mm}$ at baseline to $80 \pm 20 \mathrm{~mm}$ after one week (pain reduction $2.5 \%$ ).

\section{Participant-reported change in health-related quality of life}

Dailey 2013 reported that fatigue with movement during the six-minute walk test was significantly reduced for active TENS compared with placebo TENS $(P<0.05)$ but not for fatigue at rest. There were no significant differences between any groups for range of motion, single leg stance, sit-to-stand test, or six-minute walk test.

Lauretti 2013 reported that quality of sleep was improved in dualsite TENS (10 participants) and single-site TENS (eight participants) and worsened in the placebo TENS group (four participants, $\mathrm{P}<$ 0.05). 'Quality of fatigue' was improved in dual-site TENS (seven participants) and single-site TENS (five participants) but not in the placebo TENS group (zero participants, $P<0.05$ ). However, as quality of fatigue was not defined, it was unclear whether this meant that there was an improvement in level of fatigue.

\section{Withdrawals}

Dailey 2013 reported two withdrawals after the no-TENS intervention (without reasons) resulting in missing data from the TENS and placebo TENS interventions.

Lauretti 2013 reported two withdrawals from the placebo TENS group and one withdrawal from the TENS group due to absence of symptom relief.

\section{Adverse events}

Lauretti 2013 reported that two participants experienced muscle soreness following 70 minutes of TENS and six participants experienced gastric discomfort which they attributed to oral diclofenac (placebo TENS: four participants; single-site TENS: two participants). The authors stated that no adverse events were observed due to active TENS.
Neither of the included studies reported any serious adverse events.

\section{Specific adverse events}

Lauretti 2013 reported that two participants experienced muscle soreness following 70 minutes of TENS.

\section{Disability-related or mental health-related outcome}

Neither study reported disability-related or mental health-related outcomes.

\section{TENS compared with no treatment or waiting list control}

One study included a comparison of TENS against no treatment (Dailey 2013). We judged the quality of the evidence using GRADE to be very low; we downgraded three times due to a lack of data (Summary of findings 2).

\section{Primary outcomes}

The study did not measure primary outcomes as dichotomous data (responder analysis).

\section{Participant-reported pain relief of $30 \%$ or greater}

The study did not report participant-reported pain relief of $30 \%$ or greater.

\section{Participant-reported pain relief of $\mathbf{5 0 \%}$ or greater}

The study did not report participant-reported pain relief of $50 \%$ or greater.

\section{PGIC much or very much improved}

The study did not report PGIC much or very much improved.

\section{PGIC very much improved}

The study did not report PGIC very much improved.

\section{Secondary outcomes}

\section{Other pain-related outcomes}

Dailey 2013 found that a single 30-minute treatment of TENS reduced pain intensity (VAS) on movement compared with a noTENS control with the mean \pm standard error of the mean (SEM) difference in VAS scores $(100 \mathrm{~mm})$ before and during intervention being $11.1 \pm 2.6 \mathrm{~mm}(95 \% \mathrm{Cl} 5.9$ to 16.3$)$ for TENS and $2.6 \pm 2.5$ $\mathrm{mm}(95 \% \mathrm{Cl} 2.8$ to 7.5$)$ for no-TENS. There were no significant differences between TENS and no TENS for pain at rest. Dailey 2013 reported that active TENS increased pressure pain threshold to a greater extent than the no-TENS control in the cervical, lumbar, and anterior tibialis regions $(\mathrm{P}<0.05)$.

\section{Participant-reported change in health-related quality of life}

Dailey 2013 reported that fatigue with movement during the six-minute walk test was significantly reduced for active TENS compared with no TENS $(P<0.001)$ but not for fatigue at rest. There were no significant differences between groups for range of motion, single leg stance, sit-to-stand test, or six-minute walk test.

Dailey 2013 did not report adverse events.

\section{Serious adverse events}


Withdrawals

Dailey 2013 reported that there were two withdrawals after the noTENS intervention (without reasons) resulting in missing data from the TENS and no TENS interventions.

Adverse events, serious adverse events, specific adverse events

The study did not report adverse events.

\section{Disability-related or mental health-related outcome}

The study did not report disability-related or mental health-related outcomes.

\section{TENS added to exercise compared with exercise alone (usual care)}

Two studies included a comparison of TENS added to exercise against exercise alone (49 participants enrolled into each treatment arm for both studies combined) (Carbonario 2013; Mutlu 2013). We judged the quality of the evidence using GRADE to be very low; we downgraded three times due to a lack of data (Summary of findings 3).

\section{Primary outcomes}

\section{Participant-reported pain relief of $30 \%$ or greater}

Carbonario 2013 reported that $30 \%$ of 14 participants achieved pain relief of $30 \%$ or greater when TENS was added to an eight-week programme of aerobic and stretching exercises compared with $13 \%$ of 14 participants in the exercise alone group. These percentages equate to 4.2 participants in the TENS plus exercise group and 1.82 participants in the exercise alone group, which is illogical, and yet the authors did not describe whether they rounded their figures to the nearest whole number.

\section{Participant-reported pain relief of $\mathbf{5 0 \%}$ or greater}

The studies did not report participant-reported pain relief of $50 \%$ or greater.

\section{PGIC much or very much improved}

The studies did not report PGIC much or very much improved.

\section{PGIC very much improved}

The studies did not report PGIC very much improved.

\section{Secondary outcomes}

\section{Other pain-related outcomes}

Carbonario 2013reported greater reductions in pain intensity when TENS was added to an eight-week programme of aerobic and stretching exercises. The mean \pm SD reduction in pain intensity was $20 \pm 29 \mathrm{~mm}$ for TENS with exercise and $7.0 \pm 37 \mathrm{~mm}$ for exercise alone. There were greater increases in pressure pain threshold at tender points with TENS plus aerobic and stretching exercises.

Mutlu 2013 reported that three weeks of TENS added to the first three weeks of a 12-week supervised exercise programme produced greater than $30 \%$ reduction (relative to baseline) in the degree of tenderness at the tender points (myalgic pain score) measured using a four-point scale (no pain, mild, moderate, and severe) at the three and 12 weeks measurement time points. There were statistically significant improvements in myalgic pain score and tender point count when TENS was added to exercise compared with exercise alone.

\section{Participant-reported change in health-related quality of life}

Carbonario 2013 reported clinically important improvements in subscales for work performance, fatigue, stiffness, anxiety, and depression for TENS with exercise compared with exercise alone.

Mutlu 2013 reported that there was no additional improvements in FIQ scores or SF-36 when TENS was added to a 12-week supervised exercise programme.

\section{Withdrawals}

Carbonario 2013 reported two withdrawals and two dropouts in equal numbers from each group with no reasons.

Mutlu 2013 reported three withdrawals from each group stating that there were similar reasons for the withdrawals, although further details were not provided. In all instances missing data were removed from subsequent analysis.

\section{Adverse events, serious adverse events, specific adverse events}

Neither study reported adverse events, serious adverse events, or specific adverse events.

\section{Disability-related or mental health-related outcome}

Neither study reported disability-related or mental health-related outcomes.

\section{TENS compared with other treatment}

Four studies included a comparison of TENS versus another treatment: hydrotherapy (da Silva 2008), SAMe (Di Benedetto 1993), medication (oryzanol and amitriptyline) or electroacupuncture (Guo 2005), and superficial warmth (Lofgren 2009). We judged the quality of the evidence using GRADE to be very low; we downgraded three times due to a lack of data (Summary of findings 4).

\section{Primary outcomes}

Only one study measured primary outcomes as dichotomous data (responder analysis), but the cut-off point for response was pain relief of $25 \%$ or greater (Lofgren 2009).

\section{Participant-reported pain relief of $30 \%$ or greater}

Lofgren 2009 reported that 10/28 participants achieved pain relief of 20 units or greater on a 100 -unit NRS (i.e. pain relief of $25 \%$ ) compared with $10 / 24$ participants in the superficial warmth $\left(42^{\circ} \mathrm{C}\right)$ group with no statistically significant differences between groups.

\section{Participant-reported pain relief of $\mathbf{5 0 \%}$ or greater}

None of the studies reported participant-reported pain relief of $50 \%$ or greater.

\section{PGIC much or very much improved}

None of the studies reported PGIC much or very much improved.

PGIC very much improved

None of the studies reported PGIC very much improved. 


\section{Secondary outcomes}

\section{Other pain-related outcomes}

da Silva 2008 reported that TENS reduced pain, and improved functional outcomes and quality of life to a greater extent than hydrotherapy but treatment arms only had five participants.

Di Benedetto 1993 reported that SAMe but not TENS reduced the number of tender points. The total number of tender points reduced from 10 to five in the SAMe group.

Guo 2005 reported that TENS reduced pain and the recurrence of pain to more than routine medication (oral oryzanol and vitamin $\mathrm{B}_{1}$ (30 $\mathrm{mg}$ three times a day) and amitriptyline $(20 \mathrm{mg}$ to 30 $\mathrm{mg}$ once per day)). However, it was difficult to interpret the data analysis because pain was measured using VAS but then recategorised into discrete groups ('no effect,' 'effect,' 'remarkable effect,' and 'curing pain'). At 24-month follow-up after treatment, the number of participants that the authors described as "cured" or "having significant effects" were $1 / 14$ for TENS, 1/13 for electroacupuncture, and $1 / 2$ for medication.

Lofgren 2009 did not report other pain-related outcomes.

\section{Participant-reported change in health-related quality of life}

Di Benedetto 1993 reported that SAMe but not TENS reduced fatigue.

Lofgren 2009 reported improvements in subscales for physical function, number of days felt good after superficial warmth therapy but not TENS, with a strong tendency of the score for depression to increase (worsen) after TENS.

da Silva 2008 and Guo 2005 did not report participant-reported change in health-related quality of life.

\section{Withdrawals}

Guo 2005 reported missing data for 22 participants at six months' follow-up, 29 participants at 12 months' follow-up, and 37 participants at 24 months' follow-up, although there were no missing data at 45 days' follow-up.

Lofgren 2009 reported two withdrawals for reasons "unrelated to treatment." In addition, one participant did not complete the TENS intervention and two participants did not complete the warmth intervention. An ITT and a per-protocol analysis was conducted although how dropout data were handled was unclear.

da Silva 2008 and Di Benedetto 1993 did not report withdrawals.

\section{Adverse events, serious adverse events, specific adverse events}

Lofgren 2009 reported two adverse events: two participants reported increased pain during TENS.

da Silva 2008, Di Benedetto 1993, and Guo 2005 did not report adverse events, serious adverse events, or specific adverse events,

\section{Disability-related or mental health-related outcome}

Di Benedetto 1993 reported that TENS produced a reduction in Hamilton Rating Scale for Anxiety score only at the end of treatment whereas SAMe produced reductions in scores for both the Hamilton Rating Scale for Depression and the Hamilton Rating Scale for Anxiety by day 14 of treatment. da Silva 2008, Guo 2005, and Lofgren 2009 did not report disabilityrelated or mental health-related outcomes.

\section{DISCUSSION}

\section{Summary of main results}

The objective of this review was to assess the analgesic efficacy and adverse events of TENS for fibromyalgia in adults. We included seven RCTs and one quasi-RCT with 315 adults (299 women) and treatment sample sizes between five and 43. No studies measured participant-reported pain relief of $30 \%$ or greater as a dichotomous outcome and no studies measured PGIC, both set as primary outcomes in our review. Seven studies concluded that TENS was effective at relieving pain associated with fibromyalgia but we have very little confidence in these conclusions because sample sizes were too small. There were too few data, and outcomes were not suitably homogeneous, for any meaningful statistical pooling of results. Small studies are known to be at high risk of producing inaccurate estimates (Moore 1998) and small-study effects distort the findings of meta-analyses (AlBalawi 2013; Nüesch 2010), with stronger effect estimates in meta-analyses for smallsized studies compared with larger studies (Dechartres 2013). Estimates of efficacy are only credible when data arise from large studies or from pooling multiple moderately sized studies with similar methodology and comparison groups. The studies in our review did not meet these criteria. Thus, the quality of the evidence was very low when assessed using GRADE. The study with the lowest risk of bias overall was a double-blind randomised, placebocontrolled cross-over pilot study of 43 participants that found that a single 30-minute treatment of TENS reduced movement-related pain intensity and fatigue and increased pressure pain thresholds compared with placebo TENS (Dailey 2013). There were no reports of participants experiencing any serious adverse events during TENS, although in our experience, adverse events are generally poorly reported. Four specific adverse events were recorded: two participants experienced muscle soreness following 70 minutes of TENS (Lauretti 2013) and two participants reported increased pain during TENS (Mutlu 2013).

\section{Overall completeness and applicability of evidence}

There was insufficient robust evidence to support or refute the use of TENS for fibromyalgia. At face value, the methodology used in the studies appeared robust but on closer inspection study reports lacked clarity and detail. Replication of most studies would be impossible. Many study reports lacked fundamental information about pain outcomes such as whether scores were for: pain severity or some other aspect (e.g. bothersomeness); retrospective pain (over what duration) or present pain; pain at rest or on movement; generalised pain or localised pain; mean pain or worst pain. The precise instructions given to participants about how to rate pain severity were rarely reported. The timings of pain measurement varied across studies and sometimes it was not possible to ascertain whether pain outcomes were measured during or after TENS. This is crucial because studies suggest that maximum pain relief is obtained while TENS is switched on (i.e. during TENS; Johnson 2014).

There is a decline in the number of people that use TENS over time Johnson 2014 though a small proportion of people continue to use TENS for chronic pain for many years Bates 1980; Johnson 1992. For this reason, short-term studies on fibromyalgia, such 
as those included in this Cochrane Review, are of limited value when assessing TENS for chronic pain. More studies are needed to address issues such as frequency and duration of TENS treatment as a long-term intervention. Evidence from one Cochrane Review of 19 short-term studies provided tentative evidence that TENS as a stand-alone treatment reduces pain intensity over and above that seen with placebo (no current) for acute pain in adults, although small sample sizes prevented definitive conclusions (Johnson 2015a).

There was variation in the design of TENS treatments across studies and reports lacked specific details of the parameters and techniques used for TENS. Blinding is a challenge in TENS studies because participants experience a TENS sensation during treatment. The study by Dailey 2013 included in this review was an exemplar of good practice when designing, operationalising, and assessing blinding in TENS studies. They used an authentic sham TENS device that delivered a current for a short period of time before declining to zero current output. They also monitored the success of blinding post-intervention.

\section{Quality of the evidence}

The quality of the evidence was very low when assessed using GRADE, due to a lack of data. There were fewer than 400 participants for continuous data and 300 events for dichotomous data, and more than $25 \%$ of participants were included in studies with unclear or high risk of bias. There was a high risk of bias due to inadequate sample size and for blinding. There was no single study with low risk of bias for all items.

\section{Potential biases in the review process}

Review authors were not blinded from authors' names, institutions, and journal name or study results at any stage of the review process. Pairs of review authors undertook each stage of the review process independently, including robust searches of databases and a wide variety of sources, and we compared the outcomes.

\section{Agreements and disagreements with other studies or reviews}

Cochrane Reviews on TENS for specific types of pain have been inconclusive due to insufficient evidence for phantom and stump pain in adults (Johnson 2015b), and cancer pain in adults (Hurlow 2012). Superiority of TENS over placebo has been reported by non-Cochrane meta-analyses for chronic musculoskeletal pain (Johnson 2007), and osteoarthritis of the knee (Bjordal 2007). One Cochrane protocol for an overview of Cochrane Reviews for TENS for chronic pain has been published (Catley 2015), and there are published protocols for TENS for chronic neck pain (Porfírio 2015) and TENS for neuropathic pain in adults (Gibson 2017). One Cochrane Review on TENS of 19 RCTs (1346 participants) provides tentative evidence that TENS as a stand-alone treatment reduces pain intensity for acute pain in adults, although there was a high risk of bias associated with inadequate sample sizes that made definitive conclusions impossible (Johnson 2015a). The systematic review and guideline for fibromyalgia published by Winkelmann 2012 did not recommend TENS as a treatment for fibromyalgia pain due to low-quality evidence and the updated German guidelines for fibromyalgia gave a negative recommendation for TENS (Winkelmann 2017).

\section{AUTHORS' CONCLUSIONS}

\section{Implications for practice}

\section{For people with fibromyalgia}

There is no high-quality evidence to support or refute the use of transcutaneous electrical nerve stimulation (TENS for fibromyalgia. Further high-quality research is needed before conclusions can be made about the efficacy and safety of TENS.

\section{For clinicians}

Whether TENS should be considered as a potential treatment option for fibromyalgia remains a matter for debate. At present, there is insufficient evidence to support or refute the use of TENS for fibromyalgia.

\section{For policy-makers}

Our analysis of seven randomised controlled trials (RCTs) and one quasi-RCT with 315 adults found insufficient evidence to support or refute the use of TENS for fibromyalgia. Studies had a high risk of bias associated with inadequate sample sizes in treatment arms and there was variability in study methodology and TENS dosage.

\section{For funders}

The safety profile and ease of use of TENS compares favourably to alternative treatments because it can be self-administered and is inexpensive and readily available without prescription. In some countries, TENS needs to be prescribed by a healthcare practitioner to claim reimbursement from social security or health insurance companies.

\section{Implications for research}

\section{General}

This review has identified the need for high-quality research with long-term follow-up investigating the use of TENS for fibromyalgia pain. All the studies included in this review had design limitations such as inadequate sample sizes, outcome measures, and followup.

\section{Design}

As with previous Cochrane Reviews on TENS, we found a small number of inadequately powered studies with incomplete reporting of methodologies and treatment interventions. The CONSORT statement for non-pharmacological treatments should be adopted to report all aspects of study design and subsequent reporting (Boutron 2008). A comprehensive review of TENS methodologies by Bennett 2011 provides detailed criteria and operational guidelines to aid the design of future RCTs on TENS. Pain, Palliative and Supportive Care (PaPaS) guidance suggests that a sample size of 200 participants per treatment arm is necessary for a low risk of bias in RCTs so much larger sample sizes per treatment arms are needed in the future. The study by Dailey 2013 included in this review has been used to inform the design of a phase II randomised, double-blind, placebo-controlled, multicentre clinical study that plans to recruit 360 participants with fibromyalgia and a protocol for this study has been published (Noehren 2015). It is expected that the findings will be available in a future update of this review. For meta-analyses, the PaPaS template protocol for the evaluation of drugs for neuropathic pain recommends that at least 
500 participants would be needed to measure the magnitude of a treatment effect adequately for comparisons in which the number needed to treat for an additional beneficial outcome (NNTB) is four or above (Moore 1998).

To assess the effects of TENS on chronic pain long-term studies of a minimum of six weeks, but ideally more than six months, are necessary to monitor the decline in people who continue to use TENS long term. Ideally, TENS should be self-administered at home with people using TENS regularly throughout the day whenever they are in pain and for at least 30 minutes at a time whenever possible. In such studies, barriers to effective longterm TENS use should be evaluated and resolved before the start of the TENS intervention. Gladwell 2015a suggested that a learning phase should be incorporated into future studies to allow people to maximise the complex pattern of TENS usage. This could be achieved during a 'run-in' period in an enriched enrolment randomised withdrawal design whereby all participants initially receive active TENS to titrate dosage and optimise TENS technique (Moore 2013c). Participants who do not achieve benefits or experience adverse events (or both) are withdrawn from the study and the remaining participants randomised to receive active or placebo TENS to determine efficacy. Blinding of participants to active and placebo TENS becomes a challenge in such a design because participants become aware of sensations generated by TENS in the run-in phase. However, electrotherapeutic devices that do not produce sensations during stimulation are available (e.g. microcurrent) and information about them can be used in briefing information to create uncertainty about the need for TENS sensation for beneficial effects (see Johnson 2014, p. 170) This, coupled with the use of credible sham (no current) TENS devices, can effectively blind participants (Rakel 2010). Blinding can be monitored post-intervention using simple questions such as 'do you believe your TENS device was functioning properly?' (Deyo 1990). Monitoring TENS usage in home studies can be achieved using electronic monitoring devices to assure participant compliance with the recommended TENS treatment protocol and TENS usage can also be used as an outcome measure (Pallett 2014).

To ensure high-quality evidence in future studies - in addition to having an adequate sample size, adequate dose, and a credible sham device - researchers should consider measuring pain both during use of TENS and afterwards and also monitoring ongoing use of TENS. A health economic assessment would also be useful to enable a cost-benefit analysis to be produced.

\section{Measurement}

Future studies should use pain outcomes recommended by IMMPACT. The primary outcome should be the number of participants reporting at least a $30 \%$ or $50 \%$ reduction in pain intensity while TENS is switched on. Concurrent analgesia should be standardised and monitored. However, evaluating TENS using a unidimensional pain scale is likely to overlook other potential benefits such as 'distraction from pain,' alleviation of other unpleasant sensations (e.g. muscle tension or spasm), reduction in medication consumption, and improvements in function (Gladwell 2015b). These should be considered important secondary outcomes.

\section{ACKNOWLEDGEMENTS}

We wish to thank Anna Erskine from the Cochrane Pain, Palliative and Support Care Group for her support and the 'TENS for neuropathic pain' review group as we drafted comparable protocols. We are grateful to the following people: Dr SA Tirlapur and Dr CGT Vance for the constructive comments on our protocol; Priscilla Wittkopf from Leeds Beckett University for helping to screen records in Portuguese and for translating and helping to extract information and assessing risk of bias for da Silva 2008; and Dr Suzie Wang from Leeds Beckett University for helping to screen records in Mandarin.

Cochrane Review Group funding acknowledgement: this project was supported by the National Institute for Health Research, via Cochrane Infrastructure funding to the Cochrane Pain, Palliative and Supportive Care Review Group (PaPaS). The views and opinions expressed therein are those of the authors and do not necessarily reflect those of the Systematic Reviews Programme, NIHR, NHS or the Department of Health.

The protocol followed the agreed template for fibromyalgia, which was developed in collaboration with the Cochrane Musculoskeletal Group and Cochrane Neuromuscular Diseases Group. The editorial process was managed by the Cochrane Pain, Palliative and Supportive Care Group. 


\section{R E F E R E N C E S}

\section{References to studies included in this review}

\section{Carbonario 2013 \{published data only\}}

Carbonario F, Matsutani LA, Yuan SLK, Marques AP. Effectiveness of high-frequency transcutaneous electrical nerve stimulation at tender points as adjuvant therapy for patients with fibromyalgia. European Journal of Physical and Rehabilitation Medicine 2013;49:197-204.

\section{Dailey 2013 \{published data only\}}

Dailey D, Rakel B, Vance C, Lee J, Liebano R, Anand A, et al. Transcutaneous electrical nerve stimulation (TENS) reduces pain and improves function in people with fibromyalgia. Journal of Pain 2011;12:23.

* Dailey DL, Rakel BA, Vance CGT, Liebano RE, Amrit AS, Bush HM, et al. Transcutaneous electrical nerve stimulation reduces pain, fatigue and hyperalgesia while restoring central inhibition in primary fibromyalgia. Pain 2013;154:2554-62.

Vance C, Dailey D, Rakel B, Geasland K, Darghosian L, Alemo Munters $L$, et al. A novel method to obtain higher intensity TENS stimulation in clinical application. Journal of Pain 2015;1:S93.

\section{da Silva 2008 \{published data only\}}

* da Silva TFG, Suda EY, Marçulo CA, Paes FHS, Pinheiro GT. Comparison of transcutaneous electrical nerve stimulation and hydrotherapy effects on pain, flexibility and quality of life in patients with fibromyalgia [Comparação dos efeitos da estimulação elétrica nervosa transcutânea e da hidroterapia na dor, flexibilidade e qualidade de vida de pacientes com fibromialgia]. Fisioterapia e Pesquisa 2008;15(2):118-24.

\section{Di Benedetto 1993 \{published data only\}}

Di Benedetto P, Iona LG, Zidarich V. Clinical evaluation of Sadenosyl-L-methionine versus transcutaneous electrical nerve stimulation in primary fibromyalgia. Current Therapeutic Research 1993;53(2):222-9.

\section{Guo 2005 \{published data only\}}

Guo XJ, Jia J. Comparison of therapeutic effects of transcutaneous electrical nerve stimulation and electroacupuncture on fibromyalgia syndrome. Zhongguo Zhen Jiu 2003;23(11):653-4.

* Guo XJ, Jia J. Comparison of therapeutic effects on fibromyalgia syndrome between dermal-neurological electric stimulation and electric acupuncture. Chinese Journal of Clinical Rehabilitation 2005;9(46):171-3.

\section{Lauretti 2013 \{published data only\}}

Lauretti GR, Chubaci EF, Mattos AL. Efficacy of the use of two simultaneously TENS devices for fibromyalgia pain. Rheumatology International 2013;33:2117-22.

\section{Lofgren 2009 \{published data only\}}

Lofgren M, Norrbrink C. Pain relief in women with fibromyalgia: a cross-over study of superficial warmth stimulation and transcutaneous electrical nerve stimulation. Journal of Rehabilitation Medicine 2009;41(7):557-62.
Mutlu 2013 \{published data only\}

Mutlu B, Paker N, Bugdayci D, Tekdos D, Kesiktas N. Efficacy of supervised exercise combined with transcutaneous electrical nerve stimulation in women with fibromyalgia: a prospective controlled study. Rheumatology International 2013;33:649-55.

\section{References to studies excluded from this review}

Arroyo 1993 \{published data only\}

Arroyo JF, Cohen ML. Abnormal responses to electrocutaneous stimulation in fibromyalgia. Journal of Rheumatology 1993;20(11):1925-31.

\section{Ido 2003 \{published data only\}}

Ido CS, Rothenbuhler R, Janz J, Loris L. Transcutaneous nerve electrostimulation of low frequency to tender points of the juvenile fibromyalgia patients [Eletroestimulação nervosa transcutanea de baixa frequencia nos tender points dos pacientes fibromialgicos juvenis]. Revista Fisioterapia Universidade Sao Paulo 2003;10(1):1-6.

Kesiktas 2011 \{published data only\}

Kesiktas N, Karagulle Z, Erdogan N, Yazicioglu K, Yilmaz H, Paker $\mathrm{N}$. The efficacy of balneotherapy and physical modalities on the pulmonary system of patients with fibromyalgia. Journal of Back and Musculoskeletal Rehabilitation 2011;24(1):57-65.

Mutlu 2006 \{published data only\}

Mutlu B, Paker N, Yylmaz E, Soy D. Comparison of therapeutic efficacy of transcutaneous electrical nerve stimulation (TENS) and exercise program in fibromyalgia syndrome: a prospective randomized study. Annals of the Rheumatic Diseases 2006;65:649-55.

Simons 2006 \{published data only\} Simons DG, Dommerholt J. Myofascial pain syndromes - trigger points. Journal of Musculoskeletal Pain 2006;14(1):59-60.

Sunshine 1996 \{published data only\}

Sunshine W, Field TM, Quintino O, Fierro K, Kuhn C, Burman I, et al. Fibromyalgia benefits from massage therapy and transcutaneous electrical stimulation. Journal of Clinical Rheumatology 1996;2(1):18-22.

\section{References to ongoing studies}

Noehren 2015 \{unpublished data only\}

Noehren B, Dailey DL, Rakel BA, Vance CG, Zimmerman MB, Crofford LJ, et al. Effect of transcutaneous electrical nerve stimulation on pain, function, and quality of life in fibromyalgia: a double-blind randomized clinical trial. Physical Therapy 2015;95(1):129-40. 


\section{Additional references}

\section{Aarskog 2007}

Aarskog R, Johnson MI, Demmink JH, Lofthus A, Iversen V, Lopes-Martins R, et al. Is mechanical pain threshold after transcutaneous electrical nerve stimulation (TENS) increased locally and unilaterally? A randomized placebo controlled trial in healthy subjects. Physiotherapy Research International 2007;12(4):251-63.

\section{AlBalawi 2013}

AlBalawi Z, McAlister FA, Thorlund K, Wong M, Wetterslev J. Random error in cardiovascular meta-analyses: how common are false positive and false negative results?. International Journal Cardiology 2013;168(2):1102-7.

\section{APTA 2001}

American Physical Therapy Association. Guide to physical therapist practice. Physical Therapy. 2nd Edition. Vol. 81, Alexandria (VA): American Physical Therapy Association, 2001.

\section{Bates 1980}

Bates JA, Nathan PW. Transcutaneous electrical nerve stimulation for chronic pain. Anaesthesia 1980;35(8):817-22.

\section{Bennett 2007}

Bennett RM, Jones J, Turk DC, Russell IJ, Matallana L. An internet survey of 2,596 people with fibromyalgia. $B M C$ Musculoskeletal Disorders 2007;8(1):27.

\section{Bennett 2011}

Bennett MI, Hughes N, Johnson MI. Methodological quality in randomised controlled trials of transcutaneous electric nerve stimulation for pain: low fidelity may explain negative findings. Pain 2011;156(2):1226-32.

\section{Bidonde 2014}

Bidonde J, Busch AJ, Webber SC, Schachter CL, Danyliw A, Overend TJ, et al. Aquatic exercise training for fibromyalgia. Cochrane Database of Systematic Reviews 2014, Issue 10. [DOI: 10.1002/14651858.CD011336]

\section{Bidonde 2017}

Bidonde J, Busch AJ, Schachter CL, Overend TJ, Kim SY, Góes SM, et al. Aerobic exercise training for adults with fibromyalgia. Cochrane Database of Systematic Reviews 2017, Issue 6. [DOI: 10.1002/14651858.CD012700]

\section{Bjordal 2003}

Bjordal JM, Johnson MI, Ljunggreen AE. Transcutaneous electrical nerve stimulation (TENS) can reduce postoperative analgesic consumption. A meta-analysis with assessment of optimal treatment parameters for postoperative pain. European Journal of Pain 2003;7:181-8.

\section{Bjordal 2007}

Bjordal JM, Johnson MI, Lopes-Martins RA, Bogen B, Chow R, Ljunggren $A E$. Short term efficacy of physical interventions in osteoarthritis knee pain: a systematic review and meta-analysis of randomised placebo-controlled trials. BMC Musculoskeletal Disorders 2007;22(8):8-51.

\section{Boutron 2008}

Boutron I, Moher D, Altman DG, Schulz KF, Ravaud P, CONSORT Group. Extending the CONSORT statement to randomized trials of nonpharmacologic treatment: explanation and elaboration. Annals Internal Medicine 2008;148(4):295-309.

\section{Busch 2008}

Busch AJ, Schachter CL, Overend TJ, Peloso PM, Barber KA. Exercise for fibromyalgia: a systematic review. Journal of Rheumatology 2008;35(6):1130-44.

\section{Busse 2015}

Busse JW, Bartlett SJ, Dougados M, Johnston BC, Guyatt GH, Kirwan JR, et al. Optimal strategies for reporting pain in clinical trials and systematic reviews: recommendations from an OMERACT 12 workshop. Journal of Rheumatology 2015; Vol. 42, issue 10:1962-70.

\section{Catley 2015}

Catley MJ, Gibson W, Wand BM, Meads C, O'Connell NE. Transcutaneous Electrical Nerve Stimulation (TENS) for chronic pain - an overview of Cochrane reviews. Cochrane Database of Systematic Reviews 2015, Issue 10. [DOI: 10.1002/14651858.CD011890]

\section{Charlton 2005}

Charlton J. Stimulation-produced analgesia. In: Charlton J editor(s). Task Force on Professional Education. Washington (DC): IASP Press, 2005:93-6.

\section{Chen 2008}

Chen CC, Tabasam G, Johnson MI. Does the pulse frequency of transcutaneous electrical nerve stimulation (TENS) influence hypoalgesia? A systematic review of studies using experimental pain and healthy human participants. Physiotherapy 2008;94(1):11-20.

\section{Chen 2011}

Chen CC, Johnson MI. Differential frequency effects of strong non-painful transcutaneous electrical nerve stimulation (TENS) on experimentally-induced ischaemic pain in healthy human participants. Clinical Journal of Pain 2011;27(5):434-41.

\section{Chesterton 2003}

Chesterton LS, Foster NE, Wright CC, Baxter GD, Barlas P. Effects of TENS frequency, intensity and stimulation site manipulation on pressure pain thresholds in healthy human subjects. Pain 2003;106(1-2):73-80.

\section{Claydon 2008a}

Claydon LS, Chesterton LS. Does transcutaneous electrical nerve stimulation (TENS) produce 'dose' responses? A review of systematic reviews on chronic pain. Physical Therapy Reviews 2008;13(6):450-63.

\section{Claydon 2008b}

Claydon LS, Chesterton LS, Barlas P, Sim J. Effects of simultaneous dual-site TENS stimulation on experimental pain. European Journal of Pain 2008;12(6):696-704. 


\section{Claydon 2010}

Claydon LS, Chesterton LS, Johnson MI, Herbison GP, Bennett MI. Transcutaneous electrical nerve stimulation (TENS) for neuropathic pain in adults. Cochrane Database of Systematic Reviews 2010, Issue 10. [DOI: 10.1002/14651858.CD008756.pub2]

\section{Claydon 2013}

Claydon LS, Chesteron LS, Barlas P, Sim J. Effects of alternating frequency TENS on experimental pain in healthy human participants: a randomised controlled trial. Clinical Journal of Pain 2013;29(6):533-9.

\section{Dechartres 2013}

Dechartres A, Trinquart L, Boutron I, Ravaud P. Influence of trial sample size on treatment effect estimates: metaepidemiological study. BMJ 2013;346:£2304.

\section{DeSantana 2009}

DeSantana JM, da Silva LF, de Resende MA, Sluka KA. Transcutaneous electrical nerve stimulation at both high and low frequencies activates ventrolateral periaqueductal grey to decrease mechanical hyperalgesia in arthritic rats. Neuroscience 2009;163(4):1233-41.

\section{Deyo 1990}

Deyo R, Walsh N, Schoenfeld L, Ramamurthy S. Can trials of physical treatments be blinded? The example of transcutaneous electrical nerve stimulation for chronic pain. American Journal of Physical Medicine and Rehabilitation 1990;69(1):6-10.

\section{Dowswell 2009}

Dowswell T, Bedwell C, Lavender T, Neilson JP. Transcutaneous electrical nerve stimulation for pain relief in labour. Cochrane Database of Systematic Reviews 2009, Issue 2. [DOI: 10.1002/14651858.CD007214.pub2]

\section{Dworkin 2008}

Dworkin RH, Turk DC, Wyrwich KW, Beaton D, Cleeland CS, Farrar JT, et al. Interpreting the clinical importance of treatment outcomes in chronic pain clinical trials: IMMPACT recommendations. Journal of Pain 2008;9(2):105-21.

\section{Francis 2011}

Francis RP, Johnson MI. The characteristics of acupuncture-like transcutaneous electrical nerve stimulation (acupuncture-like TENS): a literature review. Acupuncture \& Electro-Therapeutics Research 2011;36(3/4):231-58.

\section{Furlan 2009}

Furlan AD, Pennick V, Bombardier C, van Tulder M. Updated method guidelines for systematic reviews in the Cochrane Back Review Group. Spine 2009;34:1929-41.

\section{Garrison 1996}

Garrison DW, Foreman RD. Effects of transcutaneous electrical nerve stimulation (TENS) on spontaneous and noxiously evoked dorsal horn activity in cats with transected spinal cords. Neuroscience Letters 1996;216(2):125-8.

\section{Gibson 2017}

Gibson W, Wand BM, O'Connell NE. Transcutaneous electrical nerve stimulation (TENS) for neuropathic pain in adults. Cochrane Database of Systematic Reviews 2017, Issue 9. [DOI: 10.1002/14651858.CD011976]

\section{Gladwell 2015a}

Gladwell PW, Badlan K, Cramp F, Palmer S. Problems, solutions, and strategies reported by users of transcutaneous electrical nerve stimulation for chronic musculoskeletal pain: qualitative exploration using patient interviews. Physical Therapy 2015;96(7):1039-48.

\section{Gladwell 2015b}

Gladwell PW, Badlan K, Cramp F, Palmer S. Direct and indirect benefits reported by users of transcutaneous electrical nerve stimulation for chronic musculoskeletal pain: qualitative exploration using patient interviews. Physical Therapy 2015;92(11):1518-28.

\section{Goldenberg 1987}

Goldenberg DL. Fibromyalgia syndrome: an emerging but controversial condition. JAMA 1987;257:2782-7.

\section{GRADEpro GDT 2015 [Computer program]}

GRADE Working Group, McMaster University. GRADEpro GDT. Hamilton (ON): GRADE Working Group, McMaster University, 2015.

\section{Guyatt 2008}

Guyatt GH, Oxman AD, Vist GE, Kunz R, Falck-Ytter Y, AlonsoCoello $P$, et al. GRADE: an emerging consensus on rating quality of evidence and strength of recommendations. $B M J$ 2008;336(7650):924-6.

\section{Guyatt 2013a}

Guyatt G, Oxman AD, Sultan S, Brozek J, Glasziou P, AlonsoCoello $P$, et al. GRADE guidelines: 11 . Making an overall rating of confidence in effect estimates for a single outcome and for all outcomes. Journal of Clinical Epidemiology 2013;66:151-7.

\section{Guyatt 2013b}

Guyatt GH, Oxman AD, Santesso N, Helfand M, Kunz R, Brozek J, et al. GRADE guidelines: 12. Preparing summary of findings tables - binary outcomes. Journal of Clinical Epidemiology 2013;66:158-72.

\section{Higgins 2011}

Higgins JPT, Green SE, editor(s). Cochrane Handbook for Systematic Reviews of Interventions Version 5.1.0 (updated March 2011). The Cochrane Collaboration, 2011. Available from handbook.cochrane.org.

\section{Houghton 2010}

Houghton P, Nussbaum E, Hoens A. Electrophysical agents - contraindications and precautions: an evidence-based approach to clinical decision making in physical therapy. Physiotherapy Canada 2010;62(5):1-80. 


\section{Hurlow 2012}

Hurlow A, Bennett MI, Robb KA, Johnson MI, Simpson KH, Oxberry SG. Transcutaneous electric nerve stimulation (TENS) for cancer pain in adults. Cochrane Database of Systematic Reviews 2012, Issue 3. [DOI: 10.1002/14651858.CD006276.pub3]

\section{Häuser 2012}

Häuser W, Jung E, Erbslöh-Möller B, Gesmann M, KühnBecker $\mathrm{H}$, Petermann F, et al. The German fibromyalgia consumer reports - a cross-sectional survey. BMC Musculoskeletal Disorders 2012;13(1):74.

\section{Johnson 1992}

Johnson MI, Ashton CH, Thompson JW. Long-term use of TENS at Newcastle Pain Relief Clinic. Journal of the Royal Society of Medicine 1992;85:267-8.

\section{Johnson 2007}

Johnson M, Martinson M. Efficacy of electrical nerve stimulation for chronic musculoskeletal pain: a meta-analysis of randomized controlled trials. Pain 2007;130(1-2):157-65.

\section{Johnson 2010}

Johnson MI, Walsh DM. Pain: continued uncertainty of TENS effectiveness for pain relief. Nature Reviews Rheumatology 2010;6(6):314-6

\section{Johnson 2011}

Johnson MI, Bjordal JM. Transcutaneous electrical nerve stimulation for the management of painful conditions: focus on neuropathic pain. Expert Review of Neurotherapeutics 2011;11(5):735-53.

\section{Johnson 2014}

Johnson MI. Transcutaneous Electrical Nerve Stimulation (TENS). Research to Support Clinical Practice. 1st Edition. Oxford(UK): Oxford University Press, 2014.

\section{Johnson 2015a}

Johnson MI, Paley CA, Howe TA, Sluka KA. Transcutaneous electrical nerve stimulation for acute pain. Cochrane Database of Systematic Reviews 2015, Issue 6. [DOI: 10.1002/14651858.CD006142.pub3]

\section{Johnson 2015b}

Johnson MI, Mulvey MR, Bagnall AM. Transcutaneous electrical nerve stimulation (TENS) for phantom pain and stump pain following amputation in adults. Cochrane Database of Systematic Reviews 2015, Issue 8. [DOI: 10.1002/14651858.CD007264.pub3]

\section{Kalra 2001}

Kalra A, Urban O, Sluka KA. Blockade of opioid receptors in rostral ventral medulla prevents anti-hyperalgesia produced by transcutaneous electrical nerve stimulation (TENS). Journal of Pharmacological and Experimental Therapeutics 2001;298(1):257-63.

\section{Khadilkar 2008}

Khadilkar A, Milne S, Brosseau L, Robinson V, Saginur M, Shea B. Transcutaneous electrical nerve stimulation
(TENS) versus placebo for chronic low-back pain. Cochrane Database of Systematic Reviews 2008, Issue 4. [DOI: 10.1002/14651858.CD003008.pub3]

\section{King 2001}

King EW, Sluka KA. The effect of varying frequency and intensity of transcutaneous electrical nerve stimulation on secondary mechanical hyperalgesia in an animal model of inflammation. Journal of Pain 2001;2:128-33.

\section{Koroschetz 2011}

Koroschetz J, Rehm SE, Gockel U, Brosz M, Freynhagen R, Tolle TR, et al. Fibromyalgia and neuropathic pain - differences and similarities. A comparison of 3057 patients with diabetic painful neuropathy and fibromyalgia. BMC Neurology 2011;11:55

\section{L'Abbé 1987}

L'Abbé KA, Detsky AS, O'Rourke K. Meta-analysis in clinical research. Annals of Internal Medicine 1987;107:224-33.

\section{Leonard 2010}

Leonard G, Goffaux P, Marchand S. Deciphering the role of endogenous opioids in high-frequency TENS using low and high doses of naloxone. Pain 2010;151(1):215-9.

\section{Leonard 2011}

Leonard G, Cloutier C, Marchand S. Reduced analgesic effect of acupuncture-like TENS but not conventional TENS in opioidtreated patients. Journal of Pain 2011;12:213-21.

\section{Liberati 2009}

Liberati A, Altman DG, Tezlaff J, Mulrow C, Gotzsche PC, loannidis JP, et al. The PRISMA statement for reporting systematic reviews and meta-analyses of studies that evaluate healthcare interventions: explanations and elaboration. $B M J$ 2009;339:b2700.

\section{Lunn 2014}

Lunn MP, Hughes RA, Wiffen PJ. Duloxetine for treating painful neuropathy, chronic pain or fibromyalgia. Cochrane Database of Systematic Reviews 2014, Issue 1. [DOI: 10.1002/14651858.CD007115.pub3]

\section{Ma 2001}

Ma YT, Sluka KA. Reduction in inflammation-induced sensitization of dorsal horn neurons by transcutaneous electrical nerve stimulation in anesthetized rats. Experimental Brain Research 2001;137(1):94-102.

\section{Macfarlane 2017}

Macfarlane GJ, Kronisch C, Dean LE, Atzeni F, Häuser W, Fluß E, et al. EULAR revised recommendations for the management of fibromyalgia. Annals of the Rheumatic Diseases 2017;76:318-28.

\section{Maeda 2007}

Maeda Y, Lisi TL, Vance CG, Sluka KA. Release of GABA and activation of GABA(a) in the spinal cord mediates the effects of TENS. Brain Research 2007;1136(1):43-50. 


\section{Melzack 1965}

Melzack R, Wall PD. Pain mechanisms: a new theory. Science 1965;150(3699):971-9.

\section{Millan 2002}

Millan MJ. Descending control of pain. Progress in Neurobiology 2002;66(6):355-474.

\section{Moore 1998}

Moore RA, Gavaghan D, Tramèr MR, Collins SL, McQuay HJ. Size is everything - large amounts of information are needed to overcome random effects in estimating direction and magnitude of treatment effects. Pain 1998;78(3):209-16.

\section{Moore 2008}

Moore RA, Barden J, Derry S, McQuay HJ. Managing potential publication bias. In: McQuay HJ, Kalso E, Moore RA editor(s). Systematic Reviews in Pain Research: Methodology Refined. Seattle (WA): IASP Press, 2008:15-24.

\section{Moore 2009}

Moore RA, Straube S, Wiffen PJ, Derry S, McQuay HJ. Pregabalin for acute and chronic pain in adults. Cochrane Database of Systematic Reviews 2009, Issue 3. [DOI: 10.1002/14651858.CD007076.pub2]

\section{Moore 2010a}

Moore RA, Eccleston C, Derry S, Wiffen P, Bell RF, Straube S, et al. "Evidence" in chronic pain - establishing best practice in the reporting of systematic reviews. Pain 2010;150(3):386-9. [DOI: 10.1016/j.pain.2010.05.011]

\section{Moore 2010b}

Moore RA, Straube S, Paine J, Phillips CJ, Derry S, McQuay HJ. Fibromyalgia: moderate and substantial pain intensity reduction predicts improvement in other outcomes and substantial quality of life gain. Pain 2010;149(2):360-4. [DOI: 10.1016/j.pain.2010.02.039]

\section{Moore 2010c}

Moore RA, Moore OA, Derry S, Peloso PM, Gammaitoni AR, Wang $\mathrm{H}$. Responder analysis for pain relief and numbers needed to treat in a meta-analysis of etoricoxib osteoarthritis trials: bridging a gap between clinical trials and clinical practice. Annals of the Rheumatic Diseases 2010;69(2):374-9. [DOI: 10.1136/ard.2009.107805]

\section{Moore 2010d}

Moore RA, Smugar SS, Wang H, Peloso PM, Gammaitoni A. Numbers-needed-to-treat analyses - do timing, dropouts, and outcome matter? Pooled analysis of two randomized, placebocontrolled chronic low back pain trials. Pain 2010;151(3):592-7. [DOI: 10.1016/j.pain.2010.07.013]

\section{Moore 2011a}

Moore RA, Wiffen PJ, Derry S, McQuay HJ. Gabapentin for chronic neuropathic pain and fibromyalgia in adults. Cochrane Database of Systematic Reviews 2011, Issue 3. [DOI: 10.1002/14651858.CD007938.pub3]

\section{Moore 2011b}

Moore RA, Straube S, Paine J, Derry S, McQuay HJ. Minimum efficacy criteria for comparisons between treatments using individual patient meta-analysis of acute pain trials: examples of etoricoxib, paracetamol, ibuprofen, and ibuprofen/ paracetamol combinations after third molar extraction. Pain 2011;152(5):982-9. [DOI: 10.1016/j.pain.2010.11.030]

\section{Moore 2011c}

Moore RA, Mhuircheartaigh RJ, Derry S, McQuay HJ. Mean analgesic consumption is inappropriate for testing analgesic efficacy in post-operative pain: analysis and alternative suggestion. European Journal of Anaesthesiology 2011;28(6):427-32. [DOI: 10.1097/EJA.0b013e328343c569]

\section{Moore 2012a}

Moore RA, Derry S, Aldington D, Cole P, Wiffen PJ. Amitriptyline for neuropathic pain and fibromyalgia in adults. Cochrane Database of Systematic Reviews 2012, Issue 12. [DOI: 10.1002/14651858.CD008242.pub2]

\section{Moore 2012b}

Moore RA, Straube S, Eccleston C, Derry S, Aldington D, Wiffen P, et al. Estimate at your peril: imputation methods for patient withdrawal can bias efficacy outcomes in chronic pain trials using responder analyses. Pain 2012;153(2):265-8. [DOI: 10.1016/j.pain.2011.10.004]

\section{Moore 2013a}

Moore A, Derry S, Eccleston C, Kalso E. Expect analgesic failure: pursue analgesic success. BMJ 2013;346:f2690.

\section{Moore 2013b}

Moore RA, Straube S, Aldington D. Pain measures and cut-offs - 'no worse than mild pain' as a simple universal outcome. Anaesthesia 2013;68(4):400-12.

\section{Moore 2013c}

Moore RA, Derry S, Wiffen PJ. Challenges in design and interpretation of chronic pain trials. British Journal of Anaesthesia 2013;111(1):38-45.

\section{Moore 2014a}

Moore RA, Derry S, Taylor RS, Straube S, Phillips CJ. The costs and consequences of adequately managed chronic non-cancer pain and chronic neuropathic pain. Pain Practice 2014;14(1):79-94.

\section{Moore 2014b}

Moore RA, Cai N, Skljarevski V, Tölle TR. Duloxetine use in chronic painful conditions - individual patient data responder analysis. European Journal of Pain 2014;18(1):67-75. [DOI: 10.1002/j.1532-2149.2013.00341.x]

\section{Moran 2011}

Moran F, Leonard T, Hawthorne S, Hughes CM, McCrumGardner E, Johnson MI, et al. Hypoalgesia in response to transcutaneous electrical nerve stimulation (TENS) depends on stimulation intensity. Journal of Pain 2011;12(8):929-35. 


\section{Nardone 1989}

Nardone A, Schieppati M. Influences of transcutaneous electrical stimulation of cutaneous and mixed nerves on subcortical and cortical somatosensory evoked potentials. Electroencephalography and Clinical Neurophysiology 1989;74(1):24-35.

\section{Nüesch 2010}

Nüesch E, Trelle S, Reichenbach S, Rutjes AW, Tschannen B, Altman DG, et al. Small study effects in meta-analyses of osteoarthritis trials: meta-epidemiological study. BMJ 2010;341:c3515.

\section{O'Brien 2010}

O'Brien EM, Staud RM, Hassinger AD, McCulloch RC, Craggs JG, Atchison JW, et al. Patient-centered perspective on treatment outcomes in chronic pain. Pain Medicine 2010;11(1):6-15. [DOI: 10.1111/j.1526-4637.2009.00685.x]

\section{Pallett 2014}

Pallett EJ, Rentowl P, Johnson MI, Watson PJ. Implementation fidelity of self-administered transcutaneous electrical nerve stimulation (TENS) in patients with chronic back pain: an observational study. Clinical Journal of Pain 2014;30:224-31.

\section{PaPaS 2012}

PaPaS author and referee guidance. papas.cochrane.org/papasdocuments (accessed 22 January 2013).

\section{Porfírio 2015}

Porfírio GJM, Martimbianco ALC, Brønfort G, Torloni MR, Riera R. Transcutaneous electrical nerve stimulation (TENS) for chronic neck pain. Cochrane Database of Systematic Reviews 2015, Issue 10. [DOI: 10.1002/14651858.CD011927]

\section{Queiroz 2013}

Queiroz LP. Worldwide epidemiology of fibromyalgia. Current Pain and Headache Reports 2013;17(8):356.

\section{Rakel 2010}

Rakel B, Cooper N, Adams HJ, Messer BR, Frey Law LA, Dannen DR, et al. A new transient sham TENS device allows for investigator blinding while delivering a true placebo treatment. Journal of Pain 2010;11(3):230-8.

\section{RevMan 2014 [Computer program]}

Nordic Cochrane Centre, The Cochrane Collaboration. Review Manager (RevMan). Version 5.3. Copenhagen: Nordic Cochrane Centre, The Cochrane Collaboration, 2014.

\section{Rutjes 2009}

Rutjes AW, Nuesch E, Sterchi R, Kalichman L, Hendriks E, Osiri M, et al. Transcutaneous electrostimulation for osteoarthritis of the knee. Cochrane Database of Systematic Reviews 2009, Issue 4. [DOI: 10.1002/14651858.CD002823.pub2]

\section{Santos 2013}

Santos CM, Fransischi JN, Lima-Paiva P, Sluka KA, Resende MA. Effect of transcutaneous electrical stimulation on nociception and edema produced by peripheral serotonin. International Journal of Neuroscience 2013;123(7):507-15.

\section{Sluka 1998}

Sluka KA, Bailey K, Bogush J, Olson R, Ricketts A. Treatment with either high or low frequency TENS reduces the secondary hyperalgesia observed after injection of kaolin and carrageenan into the knee joint. Pain 1998;77:97-102.

\section{Sluka 1999}

Sluka KA, Deacon M, Stibal A, Stissel S, Terpstra A. Spinal blockade of opioid receptors prevents the analgesia produced by TENS in arthritic rats. Journal of Pharmacological and Experimental Therapeutics 1999;289(2):280-6.

\section{Sluka 2000}

Sluka KA, Judge MA, McCoolley MM, Reveiz PM, Taylor BM. Low frequency TENS is less effective than high frequency TENS at reducing inflammation-induced hyperalgesia in morphinetolerant rats. European Journal of Pain 2000;4:185-93.

\section{Sluka 2006}

Sluka KA, Lisi TL, Westlund KN. Increased release of serotonin in the spinal cord during low, but not high, frequency transcutaneous electrical nerve stimulation in rats with joint inflammation. Archives of Physical Medicine and Rehabilitation 2006;87(8):1137-40.

\section{Sluka 2013}

Sluka KA, Bjordal JM, Marchand S, Rakel BA. What makes transcutaneous electrical nerve stimulation work? Making sense of the mixed results in the clinical literature. Physical Therapy 2013;93(10):1397-402.

\section{Somers 2009}

Somers DL, Clemente FR. Contralateral high or a combination of high-and-low frequency transcutaneous electrical nerve stimulation reduces mechanical allodynia and alters dorsal horn neurotransmitter content in neuropathic rats. Journal of Pain 2009;10(2):221-9.

\section{Straube 2008}

Straube S, Derry S, McQuay HJ, Moore RA. Enriched enrolment: definition and effects of enrichment and dose in trials of pregabalin and gabapentin in neuropathic pain. A systematic review. British Journal of Clinical Pharmacology 2008;66(2):266-75. [DOI: 10.1111/j.1365-2125.2008.03200.x]

\section{Straube 2010}

Straube S, Derry S, Moore RA, Paine J, McQuay HJ. Pregabalin in fibromyalgia - responder analysis from individual patient data. BMC Musculoskeletal Disorders 2010;11:150. [DOI: 10.1186/1471-2474-11-150]

\section{Straube 2011}

Straube S, Moore RA, Paine J, Derry S, Phillips CJ, Hallier E, et al. Interference with work in fibromyalgia: effect of treatment with pregabalin and relation to pain response. BMC Musculoskeletal Disorders 2011;12:125.

\section{Sultan 2008}

Sultan A, Gaskell H, Derry S, Moore RA. Duloxetine for painful diabetic neuropathy and fibromyalgia pain: systematic review of randomised trials. BMC Neurology 2008;8:29. 


\section{Vance 2007}

Vance CG, Radhakrishnan R, Skyba DA, Sluka KA.

Transcutaneous electrical nerve stimulation at both high and low frequencies reduces primary hyperalgesia in rats with joint inflammation in a time-dependent manner. Physical Therapy 2007;87(1):44-51.

\section{Vos 2012}

Vos T, Flaxman AD, Naghavi M, Lozano R, Michaud C, Ezzati M, et al. Years lived with disability (YLDs) for 1160 sequelae of 289 diseases and injuries 1990-2010: a systematic analysis for the Global Burden of Disease Study 2010. Lancet 2012;380:2163-96.

\section{Wiffen 2013}

Wiffen PJ, Derry S, Moore RA, Aldington D, Cole P, Rice ASC. Antiepileptic drugs for neuropathic pain and fibromyalgia - an overview of Cochrane reviews. Cochrane Database of Systematic Reviews 2013, Issue 11. [DOI: 10.1002/14651858.CD010567.pub2]

\section{Winkelmann 2012}

Winkelmann A, Häuser W, Friedel E, Moog-Egan M, Seeger D, Settan M, et al. Physiotherapy and physical therapies for fibromyalgia syndrome. Systematic review, meta-analysis and guideline [Arbeitsgemeinschaft der Wissenschaftlichen Medizinischen Fachgesellschaften]. Schmerz (Berlin, Germany) 2012;26(3):276-86

\section{Winkelmann 2017}

Winkelmann A, Bork H, Brückle W, Dexl C, Heldmann P, Henningsen $\mathrm{P}$, et al. Physiotherapy, occupational therapy and physical procedures for fibromyalgia syndrome [Physiotherapie, Ergotherapie und physikalische Verfahren beim Fibromyalgiesyndrom]. Schmerz (Berlin, Germany) 2017;31(3):255-65.

\section{Winklemann 2011}

Winklemann A, Perrot S, Schaefer C, Ryan K, Chandran A, Sadosky A, et al. Impact of fibromyalgia severity on health

\section{CHARACTERISTICS OF STUDIES}

Characteristics of included studies [ordered by study ID] economic costs: results from a European cross-sectional study. Applied Health Economics and Health Policy 2011;9:125-36.

\section{Wolfe 1990}

Wolfe F, Symthe HA, Yunus MB, Bennett RM, Bombardier C, Goldenberg DL, et al. The American College of Rheumatology 1990 criteria for the classification of fibromyalgia. Report of the Multicenter Criteria Committee. Arthritis and Rheumatism 1990;33:160-72.

\section{Wolfe 2010}

Wolfe F, Clauw DJ, Fitzcharles MA, Coldenberg DL, Katz RS, Mease $P$, et al. The American College of Rheumatology preliminary diagnostic criteria for fibromyalgia and measurement of symptom severity. Arthritis Care and Research 2010;62:600-10.

\section{Wolfe 2013}

Wolfe F, Brahler E, Hinz A, Hauser W. Fibromyalgia prevalence, somatic symptom reporting, and the dimensionality of polysymptomatic distress: results from a survey of the general population. Arthritis and Research Care 2013;645(5):777-85.

\section{Wolfe 2014}

Wolfe F, Walitt BT, Hauser W. What is fibromyalgia, how is it diagnosed and what does it really mean?. Arthritis Care and Research 2014;66(7):969-71.

\section{References to other published versions of this review Nnoaham 2008}

Nnoaham KE, Kumbang J. Transcutaneous electrical nerve stimulation (TENS) for chronic pain. Cochrane Database of Systematic Reviews 2008, Issue 3. [DOI: 10.1002/14651858.CD003222.pub3]

* Indicates the major publication for the study

\section{Carbonario 2013}

Type of study: quasi-randomised, controlled, parallel group - sequential allocation to groups.
Condition and number of participants randomised: fibromyalgia (1990 American College of Rheumatol-
ogy) 32 allocated to groups.
Groups: TENS $(n=16)$, exercise alone $(n=16)$.

Participants

Demographics: $\mathrm{n}=32$, mean age 53 years, all women.

Setting: Unit of Rehabilitation of Physical Therapy, General Hospital Pirajussara, Brazil.

Inclusion: aged $\geq 18$ years, referred to physiotherapy.

Exclusion: use of pacemaker, heart disease, other forms of chronic pain, seizures, pregnancy, or arthritis. 
Carbonario 2013 (Continued)

Withdrawal/dropouts: 4 ( 2 in each group).

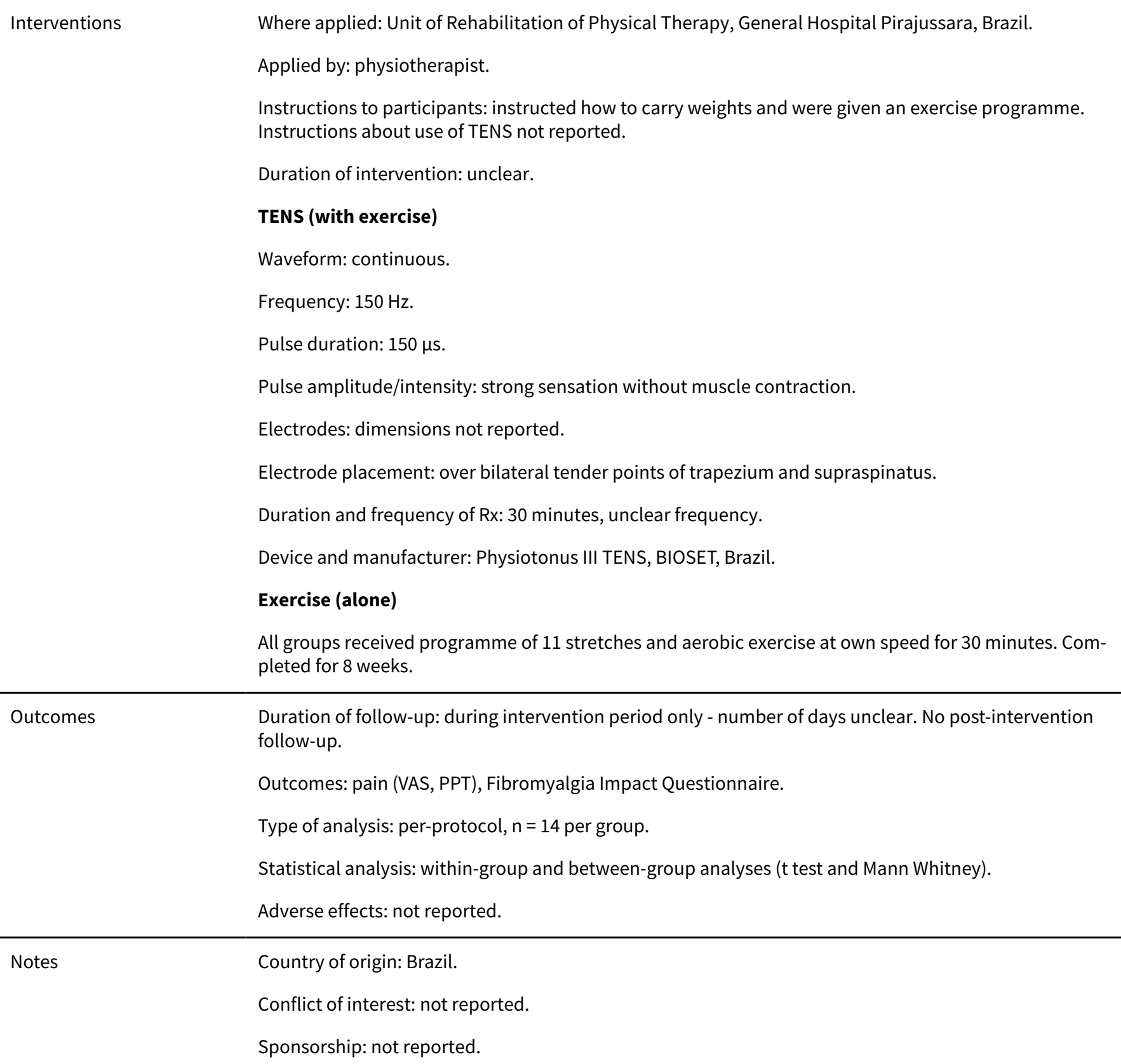

Risk of bias

Bias Authors' judgement Support for judgement

Random sequence genera- High risk Reported as sequential allocation.

tion (selection bias)

Allocation concealment High risk $\quad$ Participants consecutively allocated to each group.
(selection bias)

\begin{tabular}{|c|c|c|}
\hline $\begin{array}{l}\text { Blinding of participants } \\
\text { and personnel (perfor- } \\
\text { mance bias) }\end{array}$ & High risk & $\begin{array}{l}\text { Participants: not blind. } \\
\text { Care providers administering TENS: not blind. }\end{array}$ \\
\hline
\end{tabular}


Carbonario 2013 (Continued)

All outcomes

Blinding of outcome as-
sessment (detection bias) $\quad$ High risk $\quad$ Outcome assessors: not blind.

sessment (detection bias)

All outcomes

\section{Incomplete outcome data Unclear risk} (attrition bias)

All outcomes
Withdrawals $(n=2)$ and dropouts $(n=2)$ reported but no reasons provided. An equal number of participants withdrew from each group but no information included as to how the data were dealt with.

\section{Selective reporting (re- Unclear risk} porting bias)
No protocol registered or published. Prespecified primary outcomes for pain were reported.

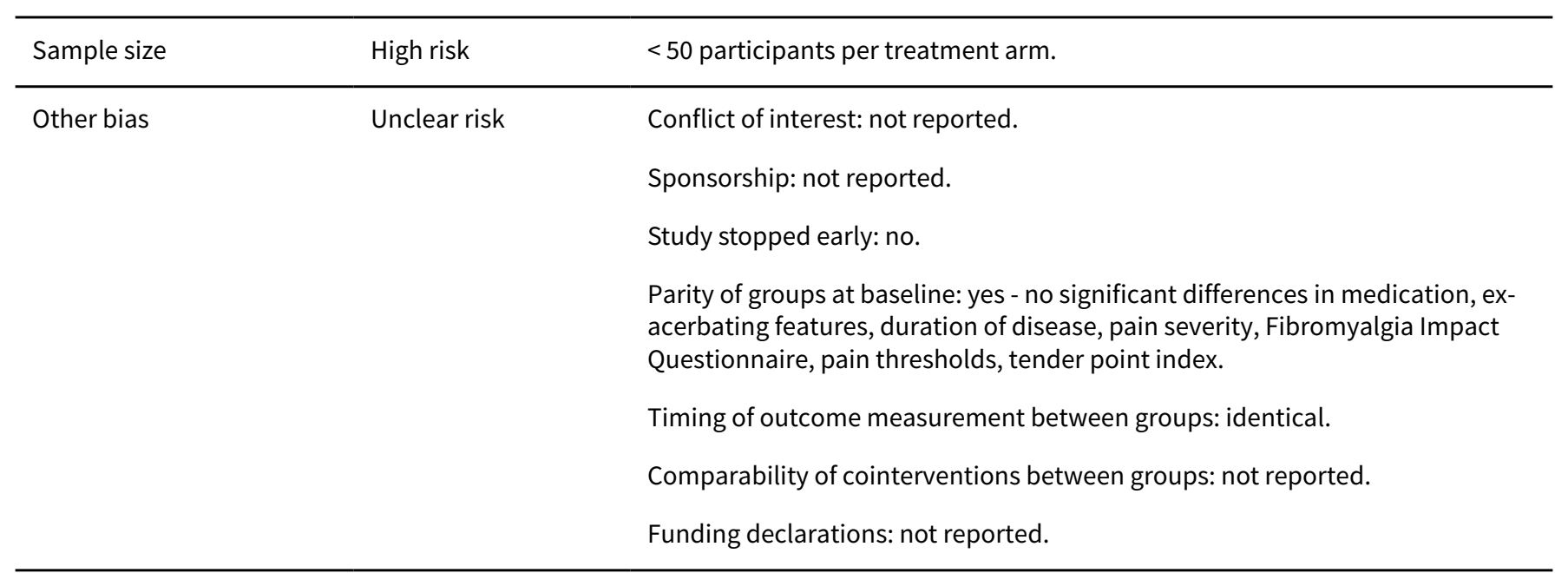

da Silva 2008

Type of study: randomised, controlled, parallel group.
Condition and number of participants randomised: fibromyalgia (1990 American College of Rheumatol-
ogy Criteria) 10 randomised.
Groups: TENS group $(n=5)$, hydrotherapy group $(n=5)$.

Participants Demographics: $\mathrm{n}=10$, mean age 48.8 years, 9 women.

Setting: Unicapital, São Paulo, Brazil.

Inclusion: diagnosis of fibromyalgia according to the American College of Rheumatology criteria.

Exclusion: neurological disease, cardiovascular disease, pacemakers, pregnant, skin disorders.

Withdrawal/dropouts: none.

Interventions Where applied: Physiotherapy Clinic of the Unicapital.

Applied by: not reported.

Instructions to participants: not reported.

Duration of intervention: 3 weeks.

\section{TENS}

Waveform: not reported. 
da Silva 2008 (Continued)

Frequency: $15 \mathrm{~Hz}$.

Pulse duration: $150 \mu \mathrm{s}$.

Pulse amplitude/intensity: constant tingling throughout the application time.

Electrodes: electrodes of surface and conductive water-based gel.

Electrode placement: bilaterally to tender points of trapezium, supraspinatus, gluts muscles, and knee medial joint line.

Duration and frequency of Rx: 3 sessions per week, 40 minutes each session, total of 10 sessions.

Device and manufacturer: Quark (Brazil).

\section{Hydrotherapy}

Exercises within pool. 5 minutes of warm-up (walking pool length and active mobilisation with flexion and extension of cervical spine, shoulder, hips, knees, and ankle); followed by20 minutes of muscle stretch $(3 \times 20 \mathrm{~s}$ at each of the following locations neck, shoulder, elbow and hand, hip, knee); followed by 15 minutes of aerobic exercise by walking with movements of upper and lower limbs.

Duration and frequency of Rx: 10 sessions, 40 minutes each session, for 3 weeks.

Pool temperature: unknown.

Duration of follow-up: during intervention period only (3 weeks). No post-intervention follow-up.
Outcome: at baseline and post-intervention; flexibility fingertip to floor test, VAS, SF-36, Nottingham
Health Profile, Beck Depression Inventory.
Type of analysis: per-protocol.
Statistical analysis: pre - post intervention (t test or Mann-Whitney). No comparison between interven-
tions.
Adverse effects: not reported.
Country of origin: Brazil.
Conflict of interest: not reported.
Sponsorship: not reported.

\section{Risk of bias}

\begin{tabular}{lll}
\hline Bias & Authors' judgement & Support for judgement \\
\hline $\begin{array}{l}\text { Random sequence genera- } \\
\text { tion (selection bias) }\end{array}$ & Unclear risk & Study reported as randomised but method not described. \\
\hline $\begin{array}{l}\text { Allocation concealment } \\
\text { (selection bias) }\end{array}$ & Unclear risk & Not reported. \\
\hline $\begin{array}{l}\text { Blinding of participants } \\
\text { and personnel (perfor- } \\
\text { mance bias) }\end{array}$ & Unclear risk & Participants: not reported. \\
$\begin{array}{l}\text { All outcomes } \\
\text { Blinding of outcome as- } \\
\text { sessment (detection bias) } \\
\text { All outcomes }\end{array}$ & Unclear risk & Care providers administering TENS: not reported. \\
\hline
\end{tabular}


da Silva 2008 (Continued)
Incomplete outcome data
Low risk
All participants completed study and there were no missing outcome data.

(attrition bias)

All outcomes

\begin{tabular}{lll}
\hline $\begin{array}{l}\text { Selective reporting (re- } \\
\text { porting bias) }\end{array}$ & Unclear risk & $\begin{array}{l}\text { No protocol registered or published. Prespecified primary outcomes for pain } \\
\text { reported. }\end{array}$
\end{tabular}

\begin{tabular}{|c|c|c|}
\hline Sample size & High risk & $<50$ participants per treatment arm. \\
\hline Other bias & Unclear risk & $\begin{array}{l}\text { Conflict of interest: not reported. } \\
\text { Sponsorship: not reported. } \\
\text { Study stopped early: no. } \\
\text { Parity of groups at baseline: no significant differences for any outcome mea- } \\
\text { sures. } \\
\text { Timing of outcome measurement between groups: identical. } \\
\text { Comparability of cointerventions between groups: not reported. } \\
\text { Funding declarations: not reported. }\end{array}$ \\
\hline
\end{tabular}

Dailey 2013

Methods Type of study: randomised, controlled, cross-over.

Condition and number of participants randomised: fibromyalgia (1990 American College of Rheumatology Criteria) 43 randomised.

Groups: active TENS ( $n=41$ analysed), placebo TENS ( $n=41$ analysed), no-TENS ( $n=43$ analysed).

Participants Demographics: $n=43$, mean age 49.2 years, 42 women.

Setting: University of lowa, IA, USA.

Inclusion: diagnosis of fibromyalgia by a physician; history of cervical or lumbar pain; stable pharmaceutical management for 1 month prior to starting the study.

Exclusion: used TENS in past 5 years, active inflammatory condition, pacemaker, pregnant, uncontrolled hypertension, significant cognitive deficits.

Withdrawal/dropouts: 2 in active TENS group, 2 in placebo TENS group, 0 in no-TENS group.

Interventions

Where applied: University Rheumatology Clinic.

Applied by: TENS assessor (unblinded). Cloth over stimulation area and TENS unit covered for all conditions.

Instructions to participants: "You will receive one of three treatments: strong sensation TENS, no-sensation TENS or no TENS. The strong-sensation TENS will feel like a twitching or tapping. The no-sensation TENS will be subtle, and you many not feel anything at all. The no TENS treatment means that a TENS unit will be attached to the electrodes but not turned on. Neither you nor the outcomes assessor will know which study treatment you are receiving."

Duration of intervention: single treatment of 30 minutes.

\section{TENS (strong sensation TENS)}

Waveform: not reported. 
Dailey 2013 (Continued)

Frequency: $100 \mathrm{~Hz}$.

Pulse duration: $200 \mu \mathrm{s}$.

Pulse amplitude/intensity: maximal tolerable intensity (mean \pm SD: $39.93 \pm 13.79 \mathrm{~mA}$ ). Adjustments made to pulse amplitude at 5-minute intervals to maintain the strong sensation of TENS.

Electrodes: butterfly-shaped electrode (Stimcare Premium Electrode; Empi, St Paul, MN, USA) dimensions not reported.

Electrode placement: participant preference for 1 of 2 locations cervical-thoracic junction $(n=19)$ or lumbo-sacral junction $(n=24)$.

Duration and frequency of Rx: once a week over 3-week period, with treatment duration of 30 minutes. Washout period of 1 week between groups/conditions.

Device/manufacturer: Rehabilicare Maxima TENS units (Empi).

\section{Placebo TENS (no-sensation TENS)}

Sham TENS device used to deliver TENS at $100 \mathrm{~Hz}, 200 \mu \mathrm{s}$ for $30 \mathrm{~s}$ after which the current amplitude was programmed to decrease (fade away) to $0 \mathrm{~mA}$ over 15 -s.

\section{No-TENS}

Electrodes attached to participant's skin but TENS device not switched on.

Outcomes

Duration of follow-up: during intervention only; 30 minutes. No post-intervention follow-up.

Outcomes: at baseline: VAS (fatigue at rest, pain at rest), 6-minute walk test (VAS fatigue with movement and pain with movement), range of motion, sit-to-stand, single leg stance, pressure pain threshold. During TENS at 30 minutes: VAS (fatigue and pain at rest) pressure pain threshold, 6-minute walk test (VAS fatigue and pain with movement), range of motion, sit-to-stand, single leg stance, conditioned pain modulation. Pressure pain threshold taken at pain site and tibialis anterior, perceived effectiveness (10-cm VAS "how effective was your treatment today?").

Blinding of the outcome assessor: "What Rx did the subject receive today - active TENS, placebo TENS, control TENS?"

Type of analysis: per protocol with $n=41$ for active and placebo TENS and $n=43$ for no-TENS group

Statistical analysis: descriptive statistics with 95\% confidence intervals. Chi ${ }^{2}$ tests used to make comparisons of categorical variables. Mixed model approach used for comparison between TENS treatment groups which accounts for the repeated measures collected through the cross-over design. To reduce the chance of a type 1 error in multiple comparisons, Tukey adjusted P values were used.

Adverse effects: not reported.

Notes

Country of origin: USA.

Conflict of interest: 2 authors had support from DJO (Vista, CA, USA).

Sponsorship: grant from American Physical Therapy Association and University of lowa. TENS units donated by company.

\section{Risk of bias}

\begin{tabular}{lll}
\hline Bias & Authors' judgement & Support for judgement \\
\hline $\begin{array}{ll}\text { Random sequence genera- } \\
\text { tion (selection bias) }\end{array}$ & Low risk & $\begin{array}{l}\text { Randomised using sequentially numbered opaque sealed envelopes which } \\
\text { were not available to the outcomes assessor. }\end{array}$ \\
\hline
\end{tabular}


Dailey 2013 (Continued)

\begin{tabular}{|c|c|c|}
\hline $\begin{array}{l}\text { Allocation concealment } \\
\text { (selection bias) }\end{array}$ & Low risk & $\begin{array}{l}\text { Order of TENS treatment randomised by drawing the order out of a hat and } \\
\text { was only available to person allocating participants to groups. }\end{array}$ \\
\hline
\end{tabular}

$\begin{array}{ll}\begin{array}{l}\text { Blinding of participants } \\ \text { and personnel (perfor- }\end{array} & \text { Participants: blind to active and placebo TENS but not to no-TENS interven- } \\ \text { mance bias) } & \text { tions. "The subject was blinded to the active TENS and placebo TENS treat- } \\ \text { All outcomes } & \text { ments." Placebo TENS administered using a device that gradually reduced } \\ & \text { current amplitude to } 0 \mathrm{~mA} \text { over a } 30 \text {-second period. This has been shown to } \\ \text { be a viable method of creating the impression of receiving active TENS (Rakel } & \text { 2010). Participants would have been aware that they were allocated to the no- } \\ \text { TENS intervention. }\end{array}$

Care providers administering TENS: not blind. The "TENS assessor" provided instructions to the participant about how to apply TENS.

\begin{tabular}{|c|c|c|}
\hline $\begin{array}{l}\text { Blinding of outcome as- } \\
\text { sessment (detection bias) } \\
\text { All outcomes }\end{array}$ & Low risk & $\begin{array}{l}\text { Outcome assessors: blind. "The outcomes assessors remained blinded to all } \\
\text { three treatments. "The no-TENS control was completed with the TENS unit } \\
\text { turned off so as to blind the outcome assessor." }\end{array}$ \\
\hline
\end{tabular}

\begin{tabular}{lll}
\hline $\begin{array}{l}\text { Incomplete outcome data } \\
\text { (attrition bias) }\end{array}$ & Unclear risk & $\begin{array}{l}\text { Withdrawals and dropouts reported but no reasons provided. Equal numbers } \\
\text { of withdrawals from TENS and placebo TENS }(n=2), \text { no withdrawals from the } \\
\text { All outcomes }\end{array}$ \\
\hline
\end{tabular}

\begin{tabular}{|c|c|c|}
\hline $\begin{array}{l}\text { Selective reporting (re- } \\
\text { porting bias) }\end{array}$ & Low risk & $\begin{array}{l}\text { Protocol registered on ClinicalTrials.gov (identifier: NCT00932360). Prespeci- } \\
\text { fied outcomes faithfully reported in study report. }\end{array}$ \\
\hline
\end{tabular}

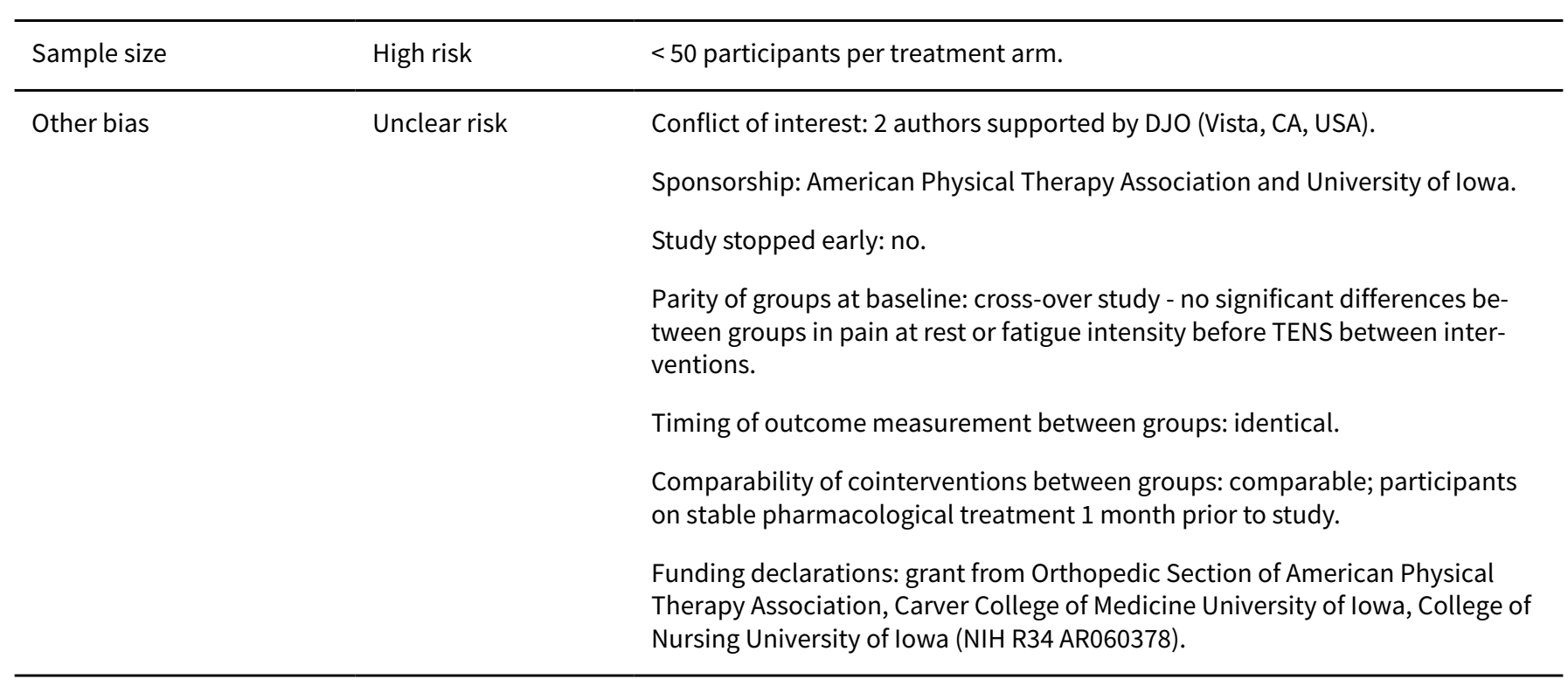

Di Benedetto 1993

\begin{tabular}{ll}
\hline Methods & Type of study: randomised, controlled, parallel group. \\
Condition and number of participants randomised: fibromyalgia (Goldenberg criteria: 30 participants). \\
Groups: TENS $(\mathrm{n}=15)$, SAMe (pharmaceutic approach) $(\mathrm{n}=15)$. \\
\hline Participants & Demographics: $\mathrm{n}=30$, aged 31-75 years (mean \pm SD: $51 \pm 9.5$ years), 29 women. \\
Setting: not reported. \\
Inclusion: participants with primary fibromyalgia using the Goldenberg criteria.
\end{tabular}


Di Benedetto 1993 (Continued)

Exclusion: associated pathological condition (e.g. rheumatoid arthritis, rheumatic diseases, hypothyroidism); severe renal, liver, or cardiovascular diseases; receiving antidepressants, corticosteroids, NSAIDs, or a combination during preceding 4 weeks.

Withdrawal/dropouts: none.

Interventions

Where applied: rehabilitation centre.

Applied by: investigator.

Instructions to participants: not reported.

Duration of intervention: 6 weeks.

\section{TENS}

Waveform: rectangular, continuous.

Frequency: $80-100 \mathrm{~Hz}$.

Pulse duration: $70 \mu \mathrm{s}$.

Pulse amplitude/intensity: minimum of a 'pleasant tingling sensation.'

Electrodes: dimensions of the electrodes not reported.

Electrode placement: at 4 tender points chosen by investigator after consultation with participant.

Duration and frequency of Rx: washout out period of 7 days where NSAIDs and neuropsychoactive drugs were not permitted. Paracetamol allowed occasionally. Then each tender point treated with TENS for 20 minutes. 5 sessions a week for 6 weeks.

Device/manufacturer: not reported.

\section{SAMe}

Washout out period of 7 days where NSAIDs and neuropsychoactive drugs were not permitted. Paracetamol allowed occasionally. After this, daily, $1 \times 200$-mg vial of SAMe intramuscularly at 8 a.m. and $2 \times$ 200-mg tablets, 1 at 12 noon and 1 at 6 p.m. for 6 weeks.

Outcome: at baseline, after washout period, after 2, 4, and 6 weeks of intervention. Pain during manual assessment of tenderness ( $0=$ no tenderness, $4=$ maximum tenderness) at C4-C5 and L4-L5, upper borders of trapezius, second costochondral junctions, lateral epicondyles, supraspinatus origins, upper outer quadrants of buttocks, and medial fat pads of knees. Total number of tender points and total tender point score (sum of all 14 individual tender point scores) computed at each time point. Pressure pain threshold at trapezius muscle and elbow measured using digital dolorimeter. Depression and anxiety scales also recorded.

Type of analysis: per protocol.

Statistical analysis: total tender point score and VAS; split plot analysis of variance and Tukey's test for multiple comparisons. Mann-Whitney for total number of tender points and each tender point score.

Adverse effects: reported no adverse effects in either group.

Country of origin: Italy.
Conflict of interest: not reported.
Sponsorship: BioResearch, Liscate, Italy.

\section{Risk of bias}


Di Benedetto 1993 (Continued)

Bias Authors' judgement Support for judgement

Random sequence genera- Unclear risk Study reported as randomised but method not described.
tion (selection bias)

\begin{tabular}{|c|c|c|}
\hline $\begin{array}{l}\text { Allocation concealment } \\
\text { (selection bias) }\end{array}$ & Unclear risk & Not described. \\
\hline
\end{tabular}

\begin{tabular}{|c|c|c|}
\hline $\begin{array}{l}\text { Blinding of participants } \\
\text { and personnel (perfor- } \\
\text { mance bias) } \\
\text { All outcomes }\end{array}$ & High risk & $\begin{array}{l}\text { Participants: not blind. } \\
\text { Care providers administering TENS: not reported. }\end{array}$ \\
\hline
\end{tabular}

\begin{tabular}{|c|c|c|}
\hline $\begin{array}{l}\text { Blinding of outcome as- } \\
\text { sessment (detection bias) } \\
\text { All outcomes }\end{array}$ & Unclear risk & Outcome assessors: not reported. \\
\hline $\begin{array}{l}\text { Incomplete outcome data } \\
\text { (attrition bias) } \\
\text { All outcomes }\end{array}$ & Low risk & All participants completed the study and there were no missing outcome data. \\
\hline $\begin{array}{l}\text { Selective reporting (re- } \\
\text { porting bias) }\end{array}$ & Unclear risk & $\begin{array}{l}\text { No protocol registered or published. Prespecified primary outcomes for pain } \\
\text { reported. Additional non-specified post-study analysis conducted. }\end{array}$ \\
\hline Sample size & High risk & $<50$ participants per treatment arm. \\
\hline Other bias & Unclear risk & $\begin{array}{l}\text { Conflict of interest: not reported. } \\
\text { Sponsorship: BioResearch, Liscate, Italy. } \\
\text { Study stopped early: no. } \\
\text { Parity of groups at baseline: yes - no significant differences in demographic or } \\
\text { pain data. } \\
\text { Timing of outcome measurement between groups: identical. } \\
\text { Comparability of cointerventions between groups: not reported; paracetamol } \\
\text { intake allowed. } \\
\text { Funding declarations: BioResearch, Liscate, Italy. }\end{array}$ \\
\hline
\end{tabular}

\section{Guo 2005}

\begin{tabular}{ll}
\hline Methods & Type of study: randomised, controlled, parallel group. \\
Condition and number of participants randomised: fibromyalgia (1990 American Society of Rheumatol- \\
ogy) 66 randomised. \\
3 groups: dermal electrical stimulation $(n=22)$, electroacupuncture $(n=22)$, medical $(n=22)$. \\
\hline Participants \\
Setting: hospital. \\
Inclusion: fibromyalgia as per diagnostic criteria from 1990 American Society of Rheumatology, sus- \\
tained pain over 3 months, minimum 11 tender points among 18 palpated points.
\end{tabular}


Exclusion: history of traumatic injury, mental disorder and rheumatoid disease, polymyalgia rheumatica, myofascial syndrome, chronic fatigue syndrome.

Withdrawal/dropouts: authors reported all participants completed study.

Interventions

Where applied: hospital.

Applied by: not reported.

Instructions to participants: not reported.

Duration of intervention: 20 days followed by 4 days' rest followed by 20-44 days.

\section{TENS (dermal electrical stimulation)}

Waveform: continuous.

Frequency: $100 \mathrm{~Hz}$ (15 minutes) $2 \mathrm{~Hz}$ (15 minutes).

Pulse duration: 6-150 ms.

Pulse amplitude/intensity: comfortable muscle contraction (mean \pm SD: $14 \pm 3 \mathrm{~mA}$ ).

Electrodes: not reported.

Electrode placement: at acupoints. 2 pairs of main points selected near most distinct tendon points: $G B$ 20, GB 21, BL 15, BL 16, BL 17, GB 54, GB30, BL 35, LI 11, LR 8, ST 15, ST 14, and ST 17. Supplementary points $\mathrm{HT} 7, \mathrm{KI} 3, \mathrm{ST} 36, \mathrm{PC} 6$.

Duration and frequency of Rx: once a day for 20 days followed by 4 days' rest then another 20 days with treatment once per day.

Device/manufacturer: Henan Xinxinng 128 Factory.

\section{Electroacupuncture}

Waveform: continuous.

Frequency: $100 \mathrm{~Hz}$ (15 minutes) $2 \mathrm{~Hz}$ (15 minutes).

Pulse duration: 6-150 ms.

Pulse amplitude/intensity: determined based on tolerance of participant.

Electrodes: application as per dermal stimulation except with No. 28 filiform needles inserted deeply. After deqi arrival the G-6085 apparatus was applied.

Duration and frequency of Rx: once a day for 20 days followed by 4 days' rest then another 20 days with treatment once per day.

Device/manufacturer: G-6085 (Qingdao Huasheng Instrument Factory).

\section{Medical group (medication)}

Oryzanol, orally, $30 \mathrm{mg} 3$ times a day, Vitamin $\mathrm{B}_{1}$ orally $30 \mathrm{mg}, 3$ times a day, Amitriptyline $10 \mathrm{mg}$ increased by $10 \mathrm{mg}$ a day to $20-30 \mathrm{mg}$ a day taken orally at night for 45 days. plete relief from symptoms to $6=$ no change in pain or other symptoms. Scores were combined to give an effectiveness rating (\%).

Type of analysis: per protocol.

Statistical analysis: $\mathrm{Chi}^{2}$ test on analgesic effects 45 days post-treatment in each group. 
Guo 2005 (Continued)

Adverse effects: not reported.

\begin{tabular}{|c|c|}
\hline \multirow[t]{2}{*}{ Notes } & $\begin{array}{l}\text { Country of origin: China. } \\
\text { Conflict of interest: not reported. }\end{array}$ \\
\hline & Sponsorship: not reported. \\
\hline
\end{tabular}

\section{Risk of bias}

\begin{tabular}{|c|c|c|}
\hline Bias & Authors' judgement & Support for judgement \\
\hline $\begin{array}{l}\text { Random sequence genera- } \\
\text { tion (selection bias) }\end{array}$ & Unclear risk & Study reported as randomised but method not described. \\
\hline $\begin{array}{l}\text { Allocation concealment } \\
\text { (selection bias) }\end{array}$ & Unclear risk & Not described. \\
\hline $\begin{array}{l}\text { Blinding of participants } \\
\text { and personnel (perfor- } \\
\text { mance bias) } \\
\text { All outcomes }\end{array}$ & High risk & $\begin{array}{l}\text { Participants: not blind. } \\
\text { Care providers administering TENS: not reported. }\end{array}$ \\
\hline
\end{tabular}

\begin{tabular}{lll}
$\begin{array}{l}\text { Blinding of outcome as- } \\
\text { sessment (detection bias) } \\
\text { All outcomes }\end{array}$ & Unclear risk & Outcome assessors: not reported. \\
\hline $\begin{array}{ll}\text { Incomplete outcome data } \\
\text { (attrition bias) }\end{array}$ & High risk & $\begin{array}{l}\text { Data missing for } 22 \text { participants at } 6 \text { months' follow-up, } 29 \text { at } 12 \text { months, and } \\
\text { All outcomes }\end{array}$ \\
\hline
\end{tabular}

Selective reporting (re- High risk porting bias)

No protocol registered or published. Prespecified primary outcomes for pain not clear. Analysis of some outcomes were not prespecified in methods. 100$\mathrm{mm}$ VAS scores converted into 4 arbitrary categories and data analysed using frequency counts.

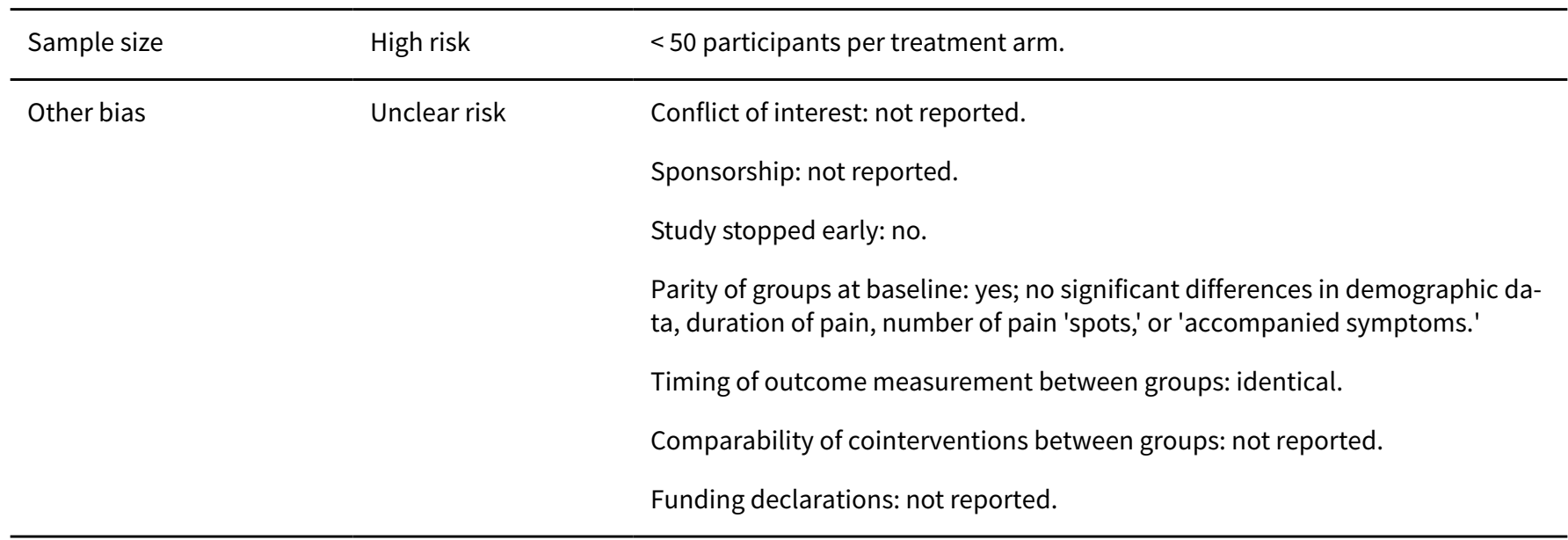

\begin{tabular}{ll}
\hline Methods & Type of study: randomised, controlled, parallel group. \\
& Condition and number of participants randomised: fibromyalgia (1990 American College of Rheumatol- \\
ogy Criteria) 39 randomised.
\end{tabular}


Lauretti 2013 (Continued)

Groups: dual-site TENS ( $n=13)$, one-site TENS $(n=13)$, placebo TENS $(n=13)$.

Participants

Demographics: $\mathrm{n}=39$, aged 18-48 years, 34 women.

Setting: University Hospital of Sao Paulo.

Inclusion: aged $\geq 18$ years, willing and able to participate, taking amitriptyline $25-50 \mathrm{mg}$ before bedtime at least 3 weeks previously as part of protocol.

Exclusion: clinically unstable disease, psychiatric disease other than depression, allergy to devices, use of beta-blockers.

Withdrawal/dropouts: 3 participants in placebo TENS group.
Where applied: hospital.

Applied by: not reported.

Instructions to participants: not reported.

Duration of intervention: 7 days.

\section{TENS}

Waveform: rectangular.

Frequency: 2 and $100 \mathrm{~Hz}$ mixed frequency.

Pulse duration: $0.2 \mathrm{~ms}$.

Pulse amplitude/intensity: $60 \mathrm{~mA}$.

Electrodes: dimensions not reported.

Electrode placement: lower back perpendicular to spine at 5th vertebra and centrally above and below the space between $\mathrm{C} 7$ and T1 spinous processes for dual-site TENS and placebo TENS. Single-site TENS was delivered at worst pain at either of these sites with placebo TENS delivered at the less painful site.

Duration and frequency of Rx: 20 minutes, at 12-hour intervals for 7 consecutive days.

Device/manufacturer: TANYX.

\section{Placebo TENS}

2 placebo TENS devices did not deliver electrical stimulation but looked similar to active device.

Outcomes

Pain outcome: pain relief (VAS $10 \mathrm{~cm}$ ); reduction in analgesic tablets; quality of sleep and fatigue.

Type of analysis: per protocol ( $n=36$ analysed).

Statistical analysis: VAS scores and daily diclofenac compared among groups during day 0 - day 7 using a 2-way ANOVA for repeated measures. Tukey's honest significant difference analysis was applied to correct $\mathrm{P}$ values for multiple group comparisons.

Adverse effects: single-site TENS; "two patients from the single-site TENS group got in sleep after the device application and complained of muscle sore due to more than 70 minutes active device application;" 4 participants in placebo group and 2 in single-site TENS group had gastric discomfort from diclofenac.

Notes Country of origin: Brazil.
Conflict of interest: not reported.




\section{Risk of bias}

\begin{tabular}{|c|c|c|}
\hline Bias & Authors' judgement & Support for judgement \\
\hline $\begin{array}{l}\text { Random sequence genera- } \\
\text { tion (selection bias) }\end{array}$ & Low risk & "Patients assigned to treatments using a randomised number generator." \\
\hline $\begin{array}{l}\text { Allocation concealment } \\
\text { (selection bias) }\end{array}$ & Unclear risk & Not described. \\
\hline $\begin{array}{l}\text { Blinding of participants } \\
\text { and personnel (perfor- } \\
\text { mance bias) } \\
\text { All outcomes }\end{array}$ & Unclear risk & $\begin{array}{l}\text { Participants: blind. Placebo (no current) TENS device was used for the placebo } \\
\text { group and for the single-TENS group and was identical to active devices. Oper- } \\
\text { ational details, including exact instructions to participants was not reported. } \\
\text { Care providers administering TENS: not reported. }\end{array}$ \\
\hline
\end{tabular}

Blinding of outcome as-
sessment (detection bias) $\quad$ Unclear risk $\quad$ Outcome assessors: not reported. Study described as "double-blind."

sessment (detection bias)

All outcomes

Incomplete outcome data Unclear risk
(attrition bias)

3/39 participants dropped out from the study due to absence of symptom relief. 2 of these dropouts were from the placebo TENS group. Missing data re-

All outcomes moved from subsequent analysis, yet these data may be related to true outcome.

\begin{tabular}{|c|c|c|}
\hline $\begin{array}{l}\text { Selective reporting (re- } \\
\text { porting bias) }\end{array}$ & Unclear risk & $\begin{array}{l}\text { No protocol registered or published. Prespecified primary outcomes for pain } \\
\text { were reported. }\end{array}$ \\
\hline
\end{tabular}

\begin{tabular}{|c|c|c|}
\hline Sample size & High risk & $<50$ participants per treatment arm. \\
\hline Other bias & Unclear risk & $\begin{array}{l}\text { Conflict of interest: not reported. } \\
\text { Sponsorship: Medecell Brasil donated active and placebo TENS devices TANYX. } \\
\text { Study stopped early: no. } \\
\text { Parity of groups at baseline: yes; no significant differences in demographic da- } \\
\text { ta, pain, or cointerventions (medication). } \\
\text { Timing of outcome measurement between groups: identical. } \\
\text { Comparability of cointerventions between groups: rescue mediation taken } \\
\text { as outcome measure; diclofenac } 50 \text { mg up to } 3 \text { times daily. Reduction in con- } \\
\text { sumption of drug medication in single-site TENS and dual-site TENS after day } \\
1 \text { of study. } \\
\text { Funding declarations: not reported; Medecell Brasil donated active and place- } \\
\text { bo TENS devices TANYX. }\end{array}$ \\
\hline
\end{tabular}

\section{Lofgren 2009}

Methods

Type of study: randomised, controlled, cross-over.

Condition and number of participants randomised: fibromyalgia (1990 American College of Rheumatology Criteria) 32 randomised. 
Setting: University Hospital, Stockholm, Sweden.

Inclusion: age 18-60 years.

Exclusion: drug misuse, serious psychiatric disease, previous experience of using TENS to alleviate musculoskeletal pain.

Withdrawal/dropouts: 2 participants for reasons not related to study.

Applied by: participants but a physiotherapist instructed the participants where to apply the electrodes.

Instructions to participants: how and where to apply the TENS electrodes.

Duration of intervention: 21 days, no washout period between interventions.

\section{TENS}

Waveform: not reported.

Frequency: $80 \mathrm{~Hz}$.

Pulse duration: not reported.

Pulse amplitude/intensity: strong but not unpleasant level.

Electrodes: 4 electrodes, $3 \times 8 \mathrm{~cm}$.

Electrode placement: at sites of pain.

Duration and frequency of Rx: daily at least 30 minutes per session, use as often as needed for 3 weeks before transfer to thermal stimulator with no washout period.

Device/manufacturer: Cefar Primo stimulator (Cefar AB, Malmö, Sweden).

\section{Superficial warmth}

Portable thermal electrical stimulation, $40 \pm 2{ }^{\circ} \mathrm{C}$, 4 electrodes $(3 \times 8 \mathrm{~cm})$, on painful sites, use for 45 minutes to 2 hours per day for 3 weeks.

Pain outcome: $0-100$ NRS (no pain to worst imaginable) before and after each treatment session. Pain diaries to report how long pain reduction lasted. Change of $\geq 20$ points (NRS) considered a responder. Fibromyalgia Impact Questionnaire.

Type of analysis: ITT and per-protocol.

Statistical analysis: between group differences (Mann Whitney test).

Adverse effects: not reported. versity Hospital and the Division of Rehabilitation Medicine. 
Lofgren 2009 (Continued)

Risk of bias

\begin{tabular}{|c|c|c|}
\hline Bias & Authors' judgement & Support for judgement \\
\hline $\begin{array}{l}\text { Random sequence genera- } \\
\text { tion (selection bias) }\end{array}$ & Unclear risk & Study reported as randomised but method not described. \\
\hline $\begin{array}{l}\text { Allocation concealment } \\
\text { (selection bias) }\end{array}$ & Unclear risk & Not described; study was cross-over design. \\
\hline $\begin{array}{l}\text { Blinding of participants } \\
\text { and personnel (perfor- } \\
\text { mance bias) } \\
\text { All outcomes }\end{array}$ & High risk & $\begin{array}{l}\text { Participants: not blind. } \\
\text { Care providers administering TENS: not blind. Physiotherapist administered } \\
\text { both treatments. }\end{array}$ \\
\hline
\end{tabular}

\begin{tabular}{|c|c|c|}
\hline $\begin{array}{l}\text { Blinding of outcome as- } \\
\text { sessment (detection bias) }\end{array}$ & High risk & $\begin{array}{l}\text { Outcome assessors: not blind (outcome assessor was the physiotherapist ad } \\
\text { ministering treatments). }\end{array}$ \\
\hline
\end{tabular}

All outcomes

\begin{tabular}{|c|c|c|}
\hline $\begin{array}{l}\text { Incomplete outcome data } \\
\text { (attrition bias) } \\
\text { All outcomes }\end{array}$ & Unclear risk & $\begin{array}{l}\text { 2/32 participants dropped out of the study for reasons "unrelated to treat- } \\
\text { ment." In addition, } 1 \text { of the remaining } 30 \text { participants did not complete TENS } \\
\text { intervention and } 2 \text { participants did not complete the warmth intervention. } \\
\text { An ITT and per-protocol analysis performed although how dropout data were } \\
\text { handled was unclear. }\end{array}$ \\
\hline
\end{tabular}

\begin{tabular}{ll}
\hline $\begin{array}{l}\text { Selective reporting (re- } \\
\text { porting bias) }\end{array}$ & $\begin{array}{l}\text { No protocol registered or published. Prespecified primary outcomes for pain } \\
\text { reported. }\end{array}$ \\
\hline
\end{tabular}

\begin{tabular}{lll}
\hline Sample size & High risk & $<50$ participants per treatment arm. \\
\hline Other bias & Unclear risk & Conflict of interest: not reported.
\end{tabular}
Sponsorship: Swedish Rheumatism Association, the Department of Rehabilita- tion Medicine, Danderyd University Hospital and the Division of Rehabilitation Medicine.

Study stopped early: no.

Parity of groups at baseline: yes - cross-over study with no significant differences in pain measures at pre-intervention time points, i.e. 'no carry-over effects detected.'

Timing of outcome measurement between groups: identical.

Comparability of cointerventions between groups: not reported.

Funding declarations: Swedish Rheumatism Association, the Department of Rehabilitation Medicine, Danderyd University Hospital and the Division of Rehabilitation Medicine. 
Mutlu 2013 (Continued)

Participants
Demographics: $n=66$, age range $32-53$ years, all women.

Setting: outpatient clinic (Hospital), Turkey.

Inclusion: fibromyalgia, women, pain duration > 1 year.

Exclusion: rheumatoid arthritis, other rheumatic disease, psychiatric disorder, antidepressant treatment, medical conditions preventing exercise training, contraindications for TENS.

Withdrawal/dropouts: 6 (3 from each group).
Where applied: hospital.

Applied by: not reported.

Instructions to participants: not reported.

Duration of intervention: 12 weeks.

\section{TENS (with exercise)}

Waveform: not reported.

Frequency: $80 \mathrm{~Hz}$.

Pulse duration: not reported.

Pulse amplitude/intensity: participant tolerance.

Electrodes: TENS applied to most painful areas (neck, shoulder, back, and hip regions).

Electrode dimensions: not reported.

Duration and frequency of Rx: once every week day in the morning for 30 minutes, for the first 3 weeks of 12-week exercise programme.

Device/manufacturer: Enraf-Nonius, En stim-4 (Netherlands).

\section{Exercise (alone)}

40-minute exercise class 3 times per week for 12 weeks that included stretching, strengthening, and aerobic exercise.

Outcomes Duration of follow-up: during intervention period only; 12 weeks. No post-intervention follow-up.

Pain outcome: baseline, 3 and 12 weeks. Tender point count scores 0 -18. Myalgic pain score (degree of the tenderness at tender points on 4-point scale $(0=$ no pain, $1=$ mild pain, $2=$ moderate pain with verbal exclamation, 3 = severe pain with withdrawal reaction). Fibromyalgia Impact Questionnaire, SF-36.

Type of analysis: per protocol $n=60(n=30$ per group) analysed.

Statistical analysis: between-group comparisons (Mann Whitney test). Within-group comparisons (Friedman 2-way ANOVA). Pearson's $\mathrm{Chi}^{2}$ tests with Yates correction were used for nominal variables.

Adverse effects: not reported. 3 participants dropped out due to increase pain ( 1 from TENS + exercise group and 2 from exercise alone group).

Notes Country of origin: Turkey.

Conflict of interest: authors declared no conflict of interest.

Sponsorship: not reported.

\section{Risk of bias}


Mutlu 2013 (Continued)

\begin{tabular}{lll} 
Bias & Authors' judgement & Support for judgement \\
\hline $\begin{array}{l}\text { Random sequence genera- } \\
\text { tion (selection bias) }\end{array}$ & Unclear risk & Study reported as binary block randomised but method not described. \\
\hline $\begin{array}{l}\text { Allocation concealment } \\
\text { (selection bias) }\end{array}$ & Unclear risk & Not described. \\
\hline $\begin{array}{l}\text { Blinding of participants } \\
\text { and personnel (perfor- } \\
\text { mance bias) }\end{array}$ & High risk & Participants: not blind. \\
All outcomes & $\begin{array}{l}\text { Care providers administering TENS: not blind. Exercise and TENS given by the } \\
\text { same physiotherapist. }\end{array}$
\end{tabular}

\begin{tabular}{ll}
\hline $\begin{array}{l}\text { Blinding of outcome as- } \\
\text { sessment (detection bias) }\end{array}$ & Low risk
\end{tabular}$\quad \begin{aligned} & \text { Outcome assessors: blind. "The physician who performs the measurements } \\
& \text { was blinded to the participant's groups." }\end{aligned}$

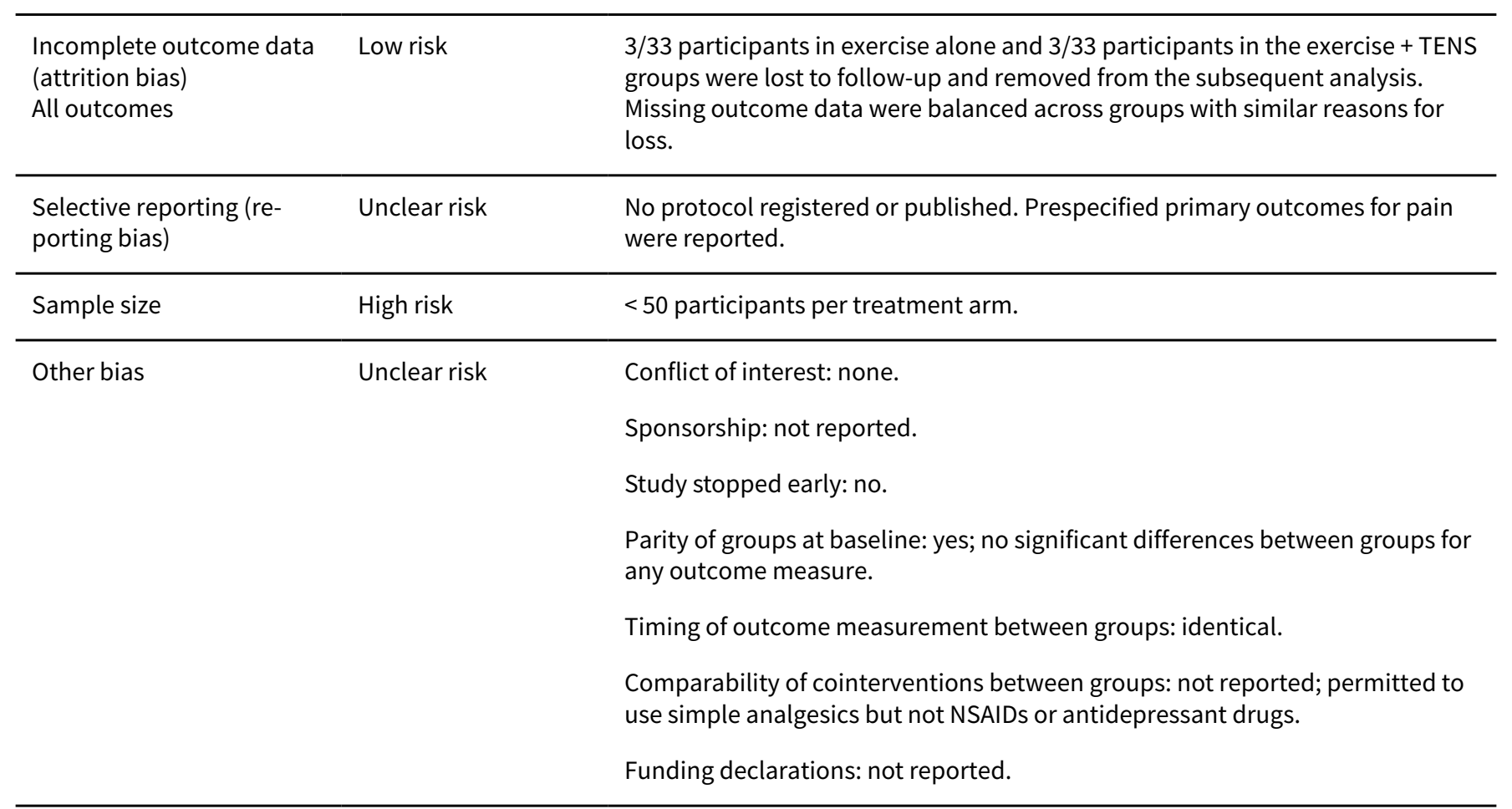

ANOVA: analysis of variance; ITT: intention-to-treat; n: number of participants; NRS: numerical rating scale; NSAID: non-steroidal antiinflammatory drug; Rx: medical prescription; s: second; SAMe: S-adenosyl-L-methionine; SF-36: 36-item Short Form; TENS: transcutaneous electrical nerve stimulation; $\mu \mathrm{s}$ : microsecond; VAS: visual analogue scale; PPT: pressure pain threshold.

Characteristics of excluded studies [ordered by study ID]

\begin{tabular}{ll}
\hline Study & Reason for exclusion \\
\hline Arroyo 1993 & Not an RCT; experimental study. \\
\hline Ido 2003 & Not an RCT; case series. \\
\hline Kesiktas 2011 & $\begin{array}{l}\text { TENS given as a combination of therapies which were given to each of the } 3 \text { arms and regarded as } 1 \\
\text { single treatment; therefore, we were unable to isolate effects of TENS from other treatment. }\end{array}$ \\
\hline
\end{tabular}




\begin{tabular}{ll}
\hline Study & Reason for exclusion \\
\hline Mutlu 2006 & Error in search citation; original source does not exist. \\
\hline Simons 2006 & $\begin{array}{l}\text { Commentaries of previously published studies. An incorrect title was tagged to this citation. The in- } \\
\text { correct title tagged to the study was "A randomised controlled study on the effect of two different } \\
\text { treatments (FREMS AND TENS) in myofascial pain syndrome," by Farina S, Casarotto M, Benelle M, } \\
\text { Tinazzi M, Fiaschi S, Goldoni M, Smania N. Europa Medicophysica 2004;40(4):293-301. Excluded be- } \\
\text { cause participants were excluded from the original study if they had clinical signs and symptoms of } \\
\text { fibromyalgia. }\end{array}$
\end{tabular}

FREMS: frequency-modulated electromagnetic neural stimulation; RCT: randomised controlled trial; TENS: transcutaneous electrical nerve stimulation.

Characteristics of ongoing studies [ordered by study ID]

Noehren 2015

\begin{tabular}{ll}
\hline Trial name or title & Fibromyalgia Activity Study with Transcutaneous Electrical Nerve Stimulation (FAST) \\
\hline Methods & Phase II randomised, double-blind, placebo-controlled multicentre clinical trial involving TENS. \\
& The initial phase of study will randomly allocate participants to receive active TENS, placebo TENS, \\
& or standard care (no TENS). After participating in the 1-month random assignment, all participants \\
& will receive active TENS for 1 month. The participants will make 4 visits to the clinic approximate- \\
ly 2 to 3.5 hours each visit. Visits will entail questionnaires, functional tasks, accelerometry, TENS, \\
pain, and fatigue assessments.
\end{tabular}

\begin{tabular}{ll}
\hline Participants & Estimated that 360 participants will be enrolled. \\
\hline Interventions & Active TENS, placebo TENS, or standard care (no TENS). \\
\hline Outcomes & $\begin{array}{l}\text { Primary aim is to test the effect of the long-term use of TENS on movement-related pain as mea- } \\
\text { sured by a numeric rating scale during 6-minute walk test in women with fibromyalgia with random } \\
\text { assignment to } 3 \text { treatments: standard care (no TENS), placebo TENS, and active TENS. }\end{array}$ \\
\hline Starting date & ClinicalTrials.gov registration June 2013. Protocol published 2015. \\
\hline Contact information & Kathleen A Sluka, PT, PhD. \\
\hline Notes & $\begin{array}{l}\text { Protocol published by Noehren } 2015 \text { is entitled "Effect of transcutaneous electrical nerve stimula- } \\
\text { tion on pain, function, and quality of life in fibromyalgia: a double-blind randomised clinical trial" } \\
\text { and the ClinicalTrials.gov identifier is NCT01888640. The study by Dailey 2013 included in this re- } \\
\text { view has been used to inform the design of this trial. }\end{array}$ \\
\hline
\end{tabular}

TENS: transcutaneous electrical nerve stimulation.

\section{ADDITIONAL TABLES}

\section{Table 1. Table of results}

\begin{tabular}{ll}
\hline Reference & Details of results for individual studies \\
\hline Carbonario 2013 & $\begin{array}{l}\text { Greater reductions in pain intensity when TENS was added to an 8-week programme of aerobic } \\
\text { and stretching exercises. A 30\% decrease in pain severity was set as a clinically relevant reduc- }\end{array}$ \\
\hline
\end{tabular}


Table 1. Table of results (Continued)

tion in pain a priori and they reported that $30 \%$ of 14 participants in the TENS with exercise group achieved this clinically relevant improvement and this was significantly greater than $13 \%$ of 14 participants in the exercise without TENS group. However, these percentages equate to 4.2 participants and 1.82 participants respectively, which is illogical. The mean \pm SD reduction in pain intensity was $20 \pm 29 \mathrm{~mm}$ for TENS and $7.0 \pm 37 \mathrm{~mm}$ for the without TENS. TENS had improvement in the Fibromyalgia Impact Questionnaire items for pain, work performance, fatigue, stiffness, anxiety, and depression and exercise had improvements for morning tiredness and depression.

Between-group comparisons not reported. Hydrotherapy group showed statistically improvement of the SF-36 total score $(P=0.007)$ and of the domains physical role $(P=0.002)$, pain $(P=0.007)$, vitality $(P=0.001)$, social aspects $(P=0.04)$, and emotional role $(P=0.005)$. There was also an improvement on the scores of the domain emotional reactions of the Nottingham Health Profile $(P$ $=0.036)$. TENS group showed statistically improvement of the SF-36 total score $(P=0.006)$ and of the domains functional capacity $(P=0.018)$, physical role $(P=0.038)$, pain $(P=0.001)$, social aspects $(P=0.02)$, and emotional role $(P=0.004)$. There was also an improvement on the scores of the Nottingham Health Profile total score $(P=0.001)$ and the domains: energy level $(P=0.009)$, pain $(P=$ $0.004)$, and emotional reactions $(P=0.03)$. There was also a significant difference on the Beck Depression Inventory $(\mathrm{P}=0.004)$. with placebo TENS with the mean \pm SD difference in VAS scores $(100 \mathrm{~mm})$ before and during intervention being $11.1 \pm 2.6 \mathrm{~mm}$ ( $95 \% \mathrm{Cl} 5.9$ to 16.3 ) for TENS and $2.3 \pm 2.6 \mathrm{~mm}(95 \% \mathrm{Cl} 2.4$ to 7.7$)$ for placebo TENS (Dailey 2013). There were no significant differences between TENS and placebo TENS for pain at rest. TENS reduced pain intensity (VAS) on movement compared with no-TENS $(2.6 \pm 2.5$ $\mathrm{mm}(95 \% \mathrm{Cl} 2.8$ to 7.5$))$. There were no significant differences between TENS and no TENS for pain at rest. PPT in the cervical region was significantly increased by active TENS versus control but not versus placebo $(P<0.05)$. Lumbar region PPT was significantly increased with active TENS versus placebo and control $(P<0.05)$. PPTs over the anterior tibialis $(n=36)$ were significantly increased with active TENS versus control $(P<0.05)$. Fatigue with movement during the 6 -minute walk test was significantly reduced for active TENS compared with placebo TENS $(P<0.005)$ and for active TENS compared with no TENS $(P<0.001)$. No significant differences between any groups for range of motion, single leg stance, sit-to-stand test, 6-minute walk test. This study had the lowest risk of bias of the studies included in our review.

The total number of tender points decreased significantly from 10 to 5 only in the SAMe group $(\mathrm{P}<$ $0.01)$ and the 2 groups differed significantly at the end of treatment $(P=0.05)$ in the total number of tender points $(P<0.05)$. The total tender point score improved significantly $(P<0.01)$ during SAMe treatment. The SAMe group also improved significantly in the subjective evaluation and psychiatric rating scales. No significant differences were shown for pain in the TENS group. Within-group statistics reported for other outcomes. SAMe produced reductions in scores for both the Hamilton Rating Scale for Depression and the Hamilton Rating Scale for Anxiety and by day 14 of treatment. TENS only produced a reduction in the Hamilton Rating Scale for Anxiety score at the end of treatment only. but both showed significantly positive differences compared with the medical group $(P<0.01)$. Data were reported for 6, 12, and 24 months. All participants completed the study. 1 week of dual-site TENS (reduction of 49.4\%) and decreased from $85 \pm 10 \mathrm{~mm}$ at baseline to $60 \pm$ $10 \mathrm{~mm}$ after 1 week of single-site TENS (pain reduction of 29\%). Placebo TENS decreased from $82 \pm$ $20 \mathrm{~mm}$ at baseline to $80 \pm 20 \mathrm{~mm}$ after 1 week (pain reduction of $2.5 \%$ ). There were statistically significant differences between groups $(P<0.02)$. Daily consumption of diclofenac $50 \mathrm{mg}$ was statistically significant between groups $(P<0.05)$ and lower for both the single- and dual-site TENS compared with placebo-TENS. Quality of sleep was improved with dual-site TENS (10 participants) and single-site TENS (8 participants) and worsened in the placebo TENS group ( $n=4 ; P<0.05)$. Quality 
Table 1. Table of results (Continued)

of fatigue was improved with dual-site TENS ( 7 participants) and single-site TENS (5 participants) but not with placebo-TENS $(n=0 ; P<0.05)$.

Lofgren 2009

Between-group differences for pain intensity were not significant. ITT analysis: 10 responders reported a reduction of $\geq 20$ points with thermal therapy and 10 responded in the same way with TENS. Per-protocol analysis: $42 \%$ of 24 participants responded to warmth and $36 \%$ of 28 responded to TENS (difference between groups was not statistically significant) $(P=0.66) .4$ participants were considered responders to both. No difference in duration of pain relief between groups.

Mutlu 2013

The exercise with TENS group showed a more significant improvement in myalgic pain at the end of the $3 r d$ week than the exercise alone group but this decrease in pain was similar in both groups by the end of week 12. Scores in the Fibromyalgia Impact Questionnaire and SF-36 improved significantly in both groups by the end of the 3rd week. 6 withdrawals reported; 3 from each group.

CI: confidence interval; SAMe: S-adenosyl-L-methionine; SD: standard deviation; SF-36: 36-item Short Form; PPT: pressure pain threshold; TENS: transcutaneous electrical nerve stimulation; VAS: visual analogue scale.

\title{
AP PE N D I C E S
}

\section{Appendix 1. Methodological considerations for chronic pain}

There have been several recent changes in how the efficacy of conventional and unconventional treatments is assessed in chronic painful conditions. The outcomes are now better defined, particularly with new criteria for what constitutes moderate or substantial benefit (Dworkin 2008); older trials may only report participants with 'any improvement.' Newer studies tend to be larger, avoiding problems from the random play of chance. Newer studies also tend to be of longer duration, up to 12 weeks, and longer studies provide a more rigorous and valid assessment of efficacy in chronic conditions. New standards have evolved for assessing efficacy in neuropathic pain, and we are now applying stricter criteria for the inclusion of studies and assessment of outcomes, and are more aware of problems that may affect our overall assessment. To summarise some of the recent insights that must be considered in this new review:

1. Pain results tend to have a U-shaped distribution rather than a bell-shaped distribution. This is true in acute pain (Moore 2011b; Moore 2011c), back pain (Moore 2010d), and arthritis (Moore 2010c), as well as in fibromyalgia (Straube 2010); in all cases, mean results usually describe the experience of almost no-one in the trial. Data expressed as means are potentially misleading, unless they can be proven to be suitable.

2. As a consequence, we have to depend on dichotomous results (the person either has or does not have the outcome) usually from pain changes or participant global assessments. The Initiative on Methods, Measurement, and Pain Assessment in Clinical Trials (IMMPACT) group has helped with their definitions of minimal, moderate, and substantial improvement (Dworkin 2008). In arthritis, studies of less than 12 weeks' duration, and especially those shorter than eight weeks, overestimate the effect of treatment (Moore 2010c); the effect is particularly strong for less-effective analgesics, and this may also be relevant in neuropathic-type pain.

3. The proportion of people with at least moderate benefit can be small, even with an effective medicine, falling from $60 \%$ with an effective medicine in arthritis to 30\% in fibromyalgia (Moore 2009; Moore 2010c; Moore 2013b; Moore 2014b; Straube 2008; Sultan 2008). One Cochrane Review of pregabalin in neuropathic pain and fibromyalgia demonstrated different response rates for different types of chronic pain (higher in diabetic neuropathy and postherpetic neuralgia and lower in central pain and fibromyalgia) (Moore 2009). This indicates that different neuropathic pain conditions should be treated separately from one another, and that pooling should not be done unless there are good grounds for doing so.

4. Individual participant analyses indicate that people who get good pain relief (moderate or better) have major benefits in many other outcomes, affecting quality of life in a significant way (Moore 2010b; Moore 2014b).

5. Imputation methods such as last observation carried forward, used when participants withdraw from clinical trials, can overstate drug efficacy especially when adverse event withdrawals with drug are greater than those with placebo (Moore 2012b).

\section{Appendix 2. Search strategies \\ CENTRAL}

\#1 MESH DESCRIPTOR TRANSCUTANEOUS ELECTRIC NERVE STIMULATION EXPLODE ALL TREES 1292

\author{
\#2 ((TENS or TNS or ENS)):TI,AB,KY 1095
}

\#3 ((Transcutaneous electric* nerve stimulation or transcutaneous nerve stimulation)):TI,AB,KY 1374 
\#4 ((Electric* nerve stimulation or electrostimulation therap* or electro-stimulation therap*)):TI,AB,KY 1496

\#5 ((Electric* nerve therap* or electroanalgesi $\left.\left.{ }^{\star}\right)\right): T I, A B, K Y 24$

\#6 \#1 OR \#2 OR \#3 OR \#4 OR \#5 2508

\#7 MESH DESCRIPTOR Fibromyalgia EXPLODE ALL TREES 616

\#8 Fibromyalgi*:TI,AB,KY 1276

\#9 Fibrositis:TI,AB,KY 60

\#10 \#7 OR \#8 OR \#9 1313

\#11 \#6 AND \#10 23

\section{MEDLINE}

1 exp TRANSCUTANEOUS ELECTRIC NERVE STIMULATION/ (6585)

2 (TENS or TNS or ENS).ti. (625)

3 (TENS or TNS or ENS).ab. (9722)

4 (Transcutaneous electric* nerve stimulation or transcutaneous nerve stimulation).mp. [mp=title, abstract, original title, name of substance word, subject heading word, keyword heading word, protocol supplementary concept word, rare disease supplementary concept word, unique identifier] (4271)

5 (Electric $^{*}$ nerve stimulation or electrostimulation therap* or electro-stimulation therap*).mp. [mp=title, abstract, original title, name of substance word, subject heading word, keyword heading word, protocol supplementary concept word, rare disease supplementary concept word, unique identifier] (4613)

6 (Electric $^{\star}$ nerve therap ${ }^{\star}$ or electroanalgesi $\left.{ }^{\star}\right) . \mathrm{mp}$. [mp=title, abstract, original title, name of substance word, subject heading word, keyword heading word, protocol supplementary concept word, rare disease supplementary concept word, unique identifier] (177)

7 or/1-6 (16370)

8 exp Fibromyalgia/ (6940)

9 Fibromyalgi\$.tw. (6932)

10 Fibrositis.tw. (419)

11 or/8-10 (8279)

1211 and 7 (38)

13 randomised controlled trial.pt. (414789)

14 controlled clinical trial.pt. (90619)

15 randomized.ab. (311705)

16 placebo.ab. (158104)

17 drug therapy.fs. (1852228)

18 randomly.ab. (220170)

19 trial.ab. (322366)

20 groups.ab. (1389663)

2113 or 14 or 15 or 16 or 17 or 18 or 19 or 20 (3518483)

22 exp animals/ not humans.sh. (4236009)

2321 not $22(2997289)$ 
2412 and $23(23)$

\section{Embase}

\section{1 exp TRANSCUTANEOUS ELECTRIC NERVE STIMULATION/ (6451)}

2 (TENS or TNS or ENS).ti. (990)

3 (TENS or TNS or ENS).ab. (13947)

4 (Transcutaneous electric* nerve stimulation or transcutaneous nerve stimulation).mp. [mp=title, abstract, heading word, drug trade name, original title, device manufacturer, drug manufacturer, device trade name, keyword] (7074)

5 (Electric* nerve stimulation or electrostimulation therap* or electro-stimulation therap*).mp. [mp=title, abstract, heading word, drug trade name, original title, device manufacturer, drug manufacturer, device trade name, keyword] (14512)

6 (Electric $^{\star}$ nerve therap* or electroanalgesi $\left.^{\star}\right) . \mathrm{mp}$. [mp=title, abstract, heading word, drug trade name, original title, device manufacturer, drug manufacturer, device trade name, keyword] (372)

7 or/1-6 (31380)

8 exp Fibromyalgia/ (15524)

9 Fibromyalgi\$.tw. (12305)

10 Fibrositis.tw. (472)

11 or/8-10 (16669)

1211 and 7 (228)

13 random\$.tw. (1081045)

14 factorial\$.tw. (27590)

15 crossover $\$ . t w . ~(57286)$

16 cross over\$.tw. (25547)

17 cross-over\$.tw. (25547)

18 placebo\$.tw. (237374)

19 (doubl\$ adj blind\$).tw. (167989)

20 (singl\$ adj blind\$).tw. (17561)

21 assign\$.tw. (285981)

22 allocat\$.tw. (103689)

23 volunteer\$.tw. (206563)

24 Crossover Procedure/ (46997)

25 double-blind procedure.tw. (235)

26 Randomized Controlled Trial/ (403169)

27 Single Blind Procedure/ (22055)

28 or/13-27 (1693697)

Transcutaneous electrical nerve stimulation (TENS) for fibromyalgia in adults (Review) 
29 (animal/ or nonhuman/) not human/ (5030350)

3028 not 29 (1503865)

3112 and $30(59)$

\section{PsycINFO}

1 (TENS or TNS or ENS).ti. (122)

2 (TENS or TNS or ENS).ab. (1474)

3 (Transcutaneous electric ${ }^{\star}$ nerve stimulation or transcutaneous nerve stimulation).mp. [mp=title, abstract, heading word, table of contents, key concepts, original title, tests \& measures] (313)

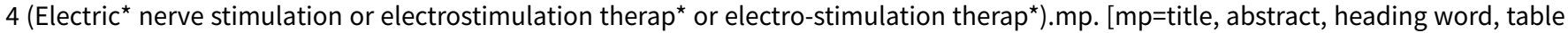
of contents, key concepts, original title, tests \& measures] (363)

5 (Electric $^{\star}$ nerve therap* ${ }^{\star}$ or electroanalgesi $\left.{ }^{\star}\right) \cdot \mathrm{mp}$. [mp=title, abstract, heading word, table of contents, key concepts, original title, tests \& measures] (13)

6 exp Fibromyalgia/ (1517)

7 Fibromyalgi\$.tw. (2633)

8 Fibrositis.tw. (39)

9 or/6-8 (2681)

10 or/1-5 (1664)

119 and $10(9)$

\section{AMED}

1 exp TRANSCUTANEOUS ELECTRIC NERVE STIMULATION/ (654)

2 (TENS or TNS or ENS).ti. (222)

3 (TENS or TNS or ENS).ab. (319)

4 (Transcutaneous electric* nerve stimulation or transcutaneous nerve stimulation).mp. [mp=abstract, heading words, title] (755)

5 (Electric ${ }^{*}$ nerve stimulation or electrostimulation therap* or electro-stimulation therap*).mp. [mp=abstract, heading words, title] (768)

6 (Electric $^{\star}$ nerve therap* or electroanalgesi $\left.{ }^{\star}\right) \cdot \mathrm{mp}$. [mp=abstract, heading words, title] (9)

7 or/1-6 (843)

8 exp Fibromyalgia/ (1611)

9 Fibromyalgi\$.tw. (1797)

10 Fibrositis.tw. (20)

11 or/8-10 (1802)

1211 and $7(8)$

\section{CINAHL}

S21 S11 AND S20

S20 S12 OR S13 OR S14 OR S15 OR S16 OR S17 OR S18 OR S19

S19 (allocat* ${ }^{\star}$ andom*)

S18 (MH "Quantitative Studies")

S17 (MH "Placebos")

S16 placebo*

S15 (random* allocat*)

S14 (MH "Random Assignment")

S13 (Randomi?ed control ${ }^{\star}$ trial $^{\star}$ )

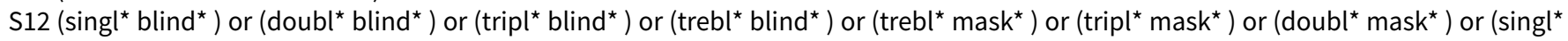
mask $\left.^{*}\right)$

S11 S6 AND S10

S10 S7 OR S8 OR S9

S9 Fibrositis

S8 Fibromyalgi*

S7 (MH "Fibromyalgia")

S6 S1 OR S2 OR S3 OR S4 OR S5

S5 (Electric* nerve therap* or electroanalgesi ${ }^{\star}$ )

S4 (Electric* nerve stimulation or electrostimulation therap* or electro-stimulation therap*)

S3 (Transcutaneous electric* nerve stimulation or transcutaneous nerve stimulation)

S2 TI ( (TENS or TNS or ENS) ) OR AB ( (TENS or TNS or ENS) )

S1 (MH "Transcutaneous Electric Nerve Stimulation")

\section{Web of Science}

Transcutaneous electrical nerve stimulation (TENS) for fibromyalgia in adults (Review) 
\#14 \#13 AND \#9

DocType=All document types; Language=All languages;

\#13 \#12 OR \#11 OR \#10

DocType=All document types; Language=All languages;

\#12 TOPIC: ((((singl* OR doubl* OR trebl* OR tripl*) SAME (blind* OR mask*))))

DocType=All document types; Language=All languages;

\#11 TOPIC: (((controlled clinical trial OR controlled trial OR clinical trial OR placebo)))

DocType=All document types; Language=All languages;

\#10 TOPIC: (( (randomised OR randomized OR randomly OR random order OR random sequence OR random allocation OR randomly allocated $\mathrm{OR}$ at random $\mathrm{OR}$ randomized controlled trial)))

DocType=All document types; Language=All languages;

\#9 \#8 AND \#3

DocType=All document types; Language=All languages;

\#8 \#7 OR \#6 OR \#5 OR \#4

DocType=All document types; Language=All languages;

\#7 TOPIC: (("Electric* nerve therap*" or electroanalgesi*))

DocType=All document types; Language=All languages;

\#6 TOPIC: (("Electric* nerve stimulation" or "electrostimulation therap*" or "electro-stimulation therap*"))

DocType=All document types; Language=All languages;

\#5 TOPIC: (("Transcutaneous electric* nerve stimulation" or "transcutaneous nerve stimulation"))

DocType=All document types; Language=All languages;

\#4 TOPIC: ((TENS or TNS or ENS))

DocType=All document types; Language=All languages;

\#3 \#2 OR \#1

DocType=All document types; Language=All languages;

\#2 TOPIC: (Fibrositis)

DocType=All document types; Language=All languages;

\#1 TOPIC: (Fibromyalgi*)

DocType=All document types; Language=All languages; Web of Science

\section{LILACS}

(TENS or TNS or ENS) or (Transcutaneous electric\$ nerve stimulation or transcutaneous nerve stimulation) or (Electric\$ nerve stimulation or electrostimulation therap\$ or electro-stimulation therap\$) or (Electric\$ nerve therap\$ or electroanalgesi\$) [Words] and Fibromyalgi\$ or Fibrositis [Words]

\section{PEDro}

1. Abstract \& Title:"electrical stimulation" pain

2. Therapy: electrotherapies, heat and cold

3. Problem: pain

4. Method: Clinical Trial

\section{SPORTDiscus (EBSCO)}

1. S1 and S2 and S3

2. Transcutaneous electric ${ }^{\star}$ nerve stimulation or transcutaneous nerve stimulation neoplasm*

3. Electric ${ }^{*}$ nerve stimulation or electrostimulation therap* or electro-stimulation therap*

4. Electric* nerve therap* or electroanalgesi*

5. exp Fibromyalgia/

6. Fibromyalgi\$.tw.

7. Fibrositis.tw.

WHAT'S NEW

\begin{tabular}{lll}
\hline Date & Event & Description \\
\hline 3 October 2017 & Review declared as stable & See Published notes. \\
\hline
\end{tabular}




\section{CONTRIBUTIONS OF AUTHORS}

- MIJ and LSC led the design of the review protocol. All authors contributed to the writing of the protocol.

- LSC developed the search strategy and MIJ, GJ, and CAP implemented the search strategy with the PaPaS Group's Information Specialist.

- CAP and MIJ screened articles for eligibility.

- LSC and CAP managed data and extracted data in the full review.

- GPH, CAP, GJ, and MIJ were responsible for data analysis.

- All authors completed the full review and will be responsible for updating the review in future.

\section{DECLARATIONS OF INTEREST}

MIJ: none known. MIJ is a physiologist and authored a book "TENS. Research to Clinical Practice" in 2014. He is also involved in the professional training of healthcare practitioners about the use of TENS.

LSC: none known. LSC is a qualified physiotherapist and is involved in the professional training of physiotherapists about the use of TENS.

GPH: none known.

GJ: none known.

CAP: none known. CAP is a qualified physiotherapist and is involved in the professional training of physiotherapists about the use of TENS.

\section{DIFFERENCES BETWEEN PROTOCOL AND REVIEW}

We extended the GRADE methods to include the potential to downgrade by three levels at once.

\section{N O T ES}

At publication, the authors are not aware of any potentially relevant studies likely to change the conclusions that are due to be published in the near future. Therefore, this review has now been stabilised for five years. If appropriate, we will update the review earlier if new evidence likely to change the conclusions is published, or if standards change substantially which necessitate major revisions.

\section{N DEX TERMS}

\section{Medical Subject Headings (MeSH)}

Electroacupuncture; Exercise; Fibromyalgia [ ${ }^{\star}$ therapy]; Hydrotherapy; Placebos [therapeutic use]; Randomized Controlled Trials as Topic; Transcutaneous Electric Nerve Stimulation [ ${ }^{\star}$ methods]

\section{MeSH check words}

Adult; Aged; Female; Humans; Male; Middle Aged; Young Adult 